\title{
Investigation of the segregation behaviour in nanocrystalline materials
}

\author{
Dissertation \\ zur Erlangung des Doktorgrades \\ der Mathematisch-Naturwissenschaftlichen Fakultäten \\ der Georg-August-Universität zu Göttingen
}

\author{
vorgelegt von \\ Catharina Gabriele Wille \\ aus Buchholz i.d.N.
}

Göttingen 2009 
'Now, here, you see, it takes all the running you can do, to stay in the same place. If you want to get somewhere else, you must run at least twice as fast as that!'

Lewis Carroll 



\section{Contents}

1 Introduction 3

2 Fundamentals $\quad \mathbf{5}$

2.1 Fe-Cu: a system with positive heat of mixing $\ldots \ldots \ldots \ldots \ldots$

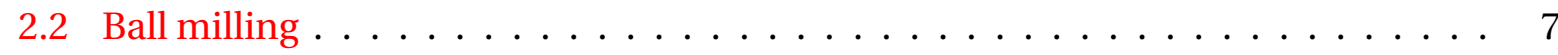

2.2 .1 Mechanism of alloying . . . . . . . . . . . . . . 9

2.2.2 Mechanisms of solid solubility extension . . . . . . . . . . . . 10

3 Experimental Methods and Techniques 13

3.1 Dual beam Focussed Ion Beam and Scanning Electron Microscopy (FIB/SEM) . . 13

3.1 .1 Operating principle . . . . . . . . . . . . . . . 13

3.1.2 Preparation of APT and TEM samples by means of FIB . . . . . . . . . . . 14

$3.2 \mathrm{X}$-Ray Diffraction $(\mathrm{XRD}) \ldots \ldots \ldots \ldots \ldots \ldots \ldots \ldots \ldots$

3.3 Transmission Electron Microscopy $(\mathrm{TEM}) \quad \ldots \ldots \ldots$. . . . . . . . . . . . . 21

3.4 Field Ion Microscopy and Atom Probe Tomography (FIM/APT) . . . . . . . . . . . 21

3.4.1 Field Ion Microscopy $($ FIM $) \ldots \ldots \ldots \ldots \ldots$. . . . . . . . . . 22

3.4 .2 Atom Probe Tomography (APT) . . . . . . . . . . . . . . 25

3.4.3 Measurement conditions . . . . . . . . . . . . . . 26

3.4 .4 Concentration depth profiles . . . . . . . . . . . . . . 26

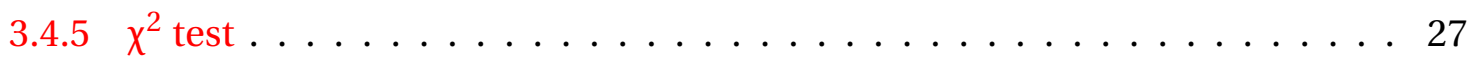

3.4 .6 Field Ion Image Tomography (FIIT) $\ldots \ldots \ldots \ldots$. . . . . . . . . 27

3.5 Differential Scanning Calorimetry (DSC) . . . . . . . . . . . . . . . . 29

4 Results and Discusssion 31

4.1 Morphology - light optical and scanning electron microscopy . . . . . . . . . 31

4.2 Diffraction study by X-Rays . . . . . . . . . . . . . . . . 37

4.3 Transmission Electron Microcroscopy . . . . . . . . . . . . . . . . . . . 47

4.4 Field Ion Microscopy and Field Ion Image Tomography $\ldots \ldots \ldots$ 
4.5 Atom Probe Tomography $\ldots \ldots \ldots \ldots$. . . . . . . . . . . 63

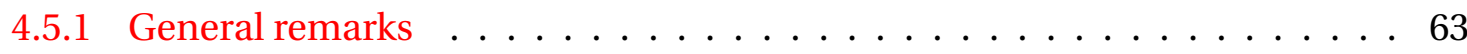

4.5 .2 Cu-rich samples: Cu-2.5at.\%Fe . . . . . . . . . . . . . . 67

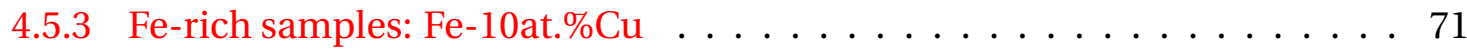

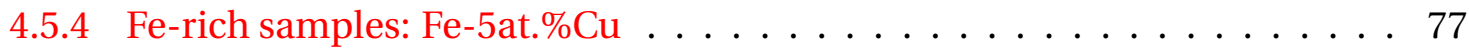

4.5.5 Fe-rich samples: Fe-2.5at.\%Cu . . . . . . . . . . . . . . 82

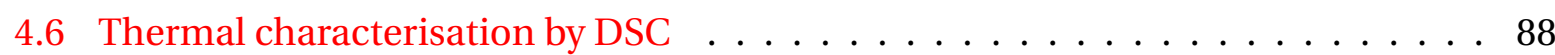

5 Conclusions $\quad 91$

6 Summary and Outlook $\quad 95$

$\begin{array}{lll}\text { Publications } & \text { IX }\end{array}$

Danksagung $\quad$ X

$\begin{array}{lll}\text { Lebenslauf } & \text { XII }\end{array}$ 


\section{Introduction}

A major challenge in modern metallurgy and materials physics is the development of new ever stronger and tougher materials. To create materials with desired properties systematically, gaining a deeper understanding of the processes underlying their production is necessary.

Many of the most promising novel materials are far off their equilibrium state, i.e. nanostructured materials or alloys with constituents that are immiscible in thermodynamic equilibrium. One of the methods used for the preparation of such meta-stable materials is mechanical alloying (MA). This technique allows for instance for the production of supersaturated solid solutions, chemically disordered intermetallics, nanocrystalline, and amorphous materials [Suryanarayana01].

For the past three decades, mechanical allloying has already been extensively employed for the mass production of meta-stable materials, as it presents a simple and cheap preparation route, which can easily be scaled up from laboratory to industrial performance. As an example, the chemical industry has made commercial quantities of nanometre sized powder particles which are ideally suited as catalysts due to their high surface area.

In this powder metallurgical process, also known as ball milling, powder particles are subjected to severe mechanical deformation and repeated fracture and cold-welding processes resulting from collisions between balls in a vial. The ball milled powders commonly show extraordinary macroscopic properties (e.g. hardness and wear resistance) owing to their nonequilibrium state and are of great interest as feedstock for consolidation to bulk-materials and other powder metallurgical processing routes.

For the time being the process of MA has to be adapted to every new material via trial and error as there is no commonly accepted model for the mixing of immiscible elements during this process and only a rough understanding of phase formation in miscible systems. Evidently, the distinction between these models can only be drawn on the nano-scale and requires sophisticated high-resolution characterisation methods. Conventional direct observation methods have a resolution limit of some nanometres.

Owing to its exceptional capability of spatially resolved, quantitative chemical analy- 
sis Atom Probe Tomography (APT) offers a unique possibility to elucidate these questions [Miller96]. Therefore, APT is predestined for the characterisation of mechanically alloyed powders. Difficulties in preparing fine needle-shaped APT specimens from powder materials, that have so far hampered APT analysis of ball milled powders, have been recently overcome by means of Focused Ion-Beam (FIB) milling [Miller05], [Choi07b] ,[Choi07a]. Due to this achievement, APT could be successfully applied to the native ball milled powders to elucidate the underlying alloying process of the Fe-Cu system.

Within the frame of this work a combination of structural characterisation by means of XRay Diffractometry (XRD) and Transmission Electron Microscopy (TEM) besides quantitative chemical analyses by means of Atom Probe Tomography (APT) yielded the optimum analysis to systematically characterise the processes involved in the production of mechanically alloyed $\mathrm{Fe}-\mathrm{Cu}$-powders.

The Fe-Cu was chosen as a model system since, exhibiting the Invar effect [Gorria06], it is among the most attractive systems for technical application. Furthermore this system is immiscible and characterised by a large positive heat of mixing. In combination with the widespread application and accessibility this predestines Fe-Cu as a binary model to elaborate the enforced nonequilibrium solubility for immiscible systems.

The theoretical background of the Fe-Cu-system and the process of ball milling will be discussed in chapter 2. Chapter 3 will introduce the employed experimental methods and techniques in due consideration of algorithms and evaluation procedures. The respective measurement conditions will be presented as well. An overview of the results obtained by the different techniques is given in chapter 4 and the results are discussed in comparison with literature. Finally the results are summarised in chapter 5 . 


\section{Fundamentals}

\subsection{Fe-Cu: a system with positive heat of mixing}

The FeCu-system has been thermodynamically well characterised. At ambient temperatures the two metals exhibit negligible mutual solubility ( $<0.1$ at.\%). Even at elevated temperatures the phase diagram shows a significant miscibility gap over the whole concentration range, see Fig. 2.1, resulting from the large positive heat of mixing $\left(\Delta \mathrm{H}_{m i x}=+13 \frac{\mathrm{kJ}}{\mathrm{mol}}\right.$ for Fe-50at.\%Cu [Miedema76]). This large heat of mixing and the non-existence of intermetallic phases predestine $\mathrm{Fe}-\mathrm{Cu}$ as a model alloy system for mechanical alloying.

The maximum solubility of $\mathrm{Fe}$ in $\mathrm{Cu}$ is 4.9 at.\% at $1096^{\circ} \mathrm{C}$ with a melting temperature of pure $\mathrm{Cu}$ of $1084.87^{\circ} \mathrm{C}$. In alpha Fe (bcc) only 1.9 at.\% Cu can be solved at most $\left(850^{\circ} \mathrm{C}\right)$, whereas 12.5 at.\% $\mathrm{Cu}$ are the solubility limit in gamma $\mathrm{Fe}$ (fcc) at $1400^{\circ} \mathrm{C}$. According to the Hume-Rotheryrules [Haasen94], the atomic radii of two elements may only differ by $15 \%$ and the difference in electro negativities by Pauli may not exceed 0.4 for the two elements to be miscible. The metallic radii of $\mathrm{Fe}$ and $\mathrm{Cu}$ are $0.124 \mathrm{~nm}$ and $0.128 \mathrm{~nm}$, respectively. This yields a deviation of less then 3\%. The electro negativities [Atkins02] of 1.8 and 1.9 do not either hinder the miscibility. But the additional third rule indicates the reason for the lacking miscibility: the crystal structures have to coincide to grant miscibility. The thermodynamically stable room temperature crystalline sturcture of Fe is the alpha bcc structure whereas $\mathrm{Cu}$ only exhibits the fcc phase at all temperatures. Hence, this discrepancy in crystal structure mainly hinders the solubity.

However, all of the above considerations only apply to a system in equilibrium. One of the most interesting aspects of ball milling is its capability to produce materials, which are far off equilibrium. For these metastable materials, the equilibrium phase diagram as discussed above is only applicable to some extent. It should rather be taken as a guide line. For the process of mechanical alloying by ball milling, the mechanical properties of the constituent elements play an additional important role. The two elements examined within this work exhibit entirely different mechanical properties, Fe being brittle and $\mathrm{Cu}$ being ductile. 


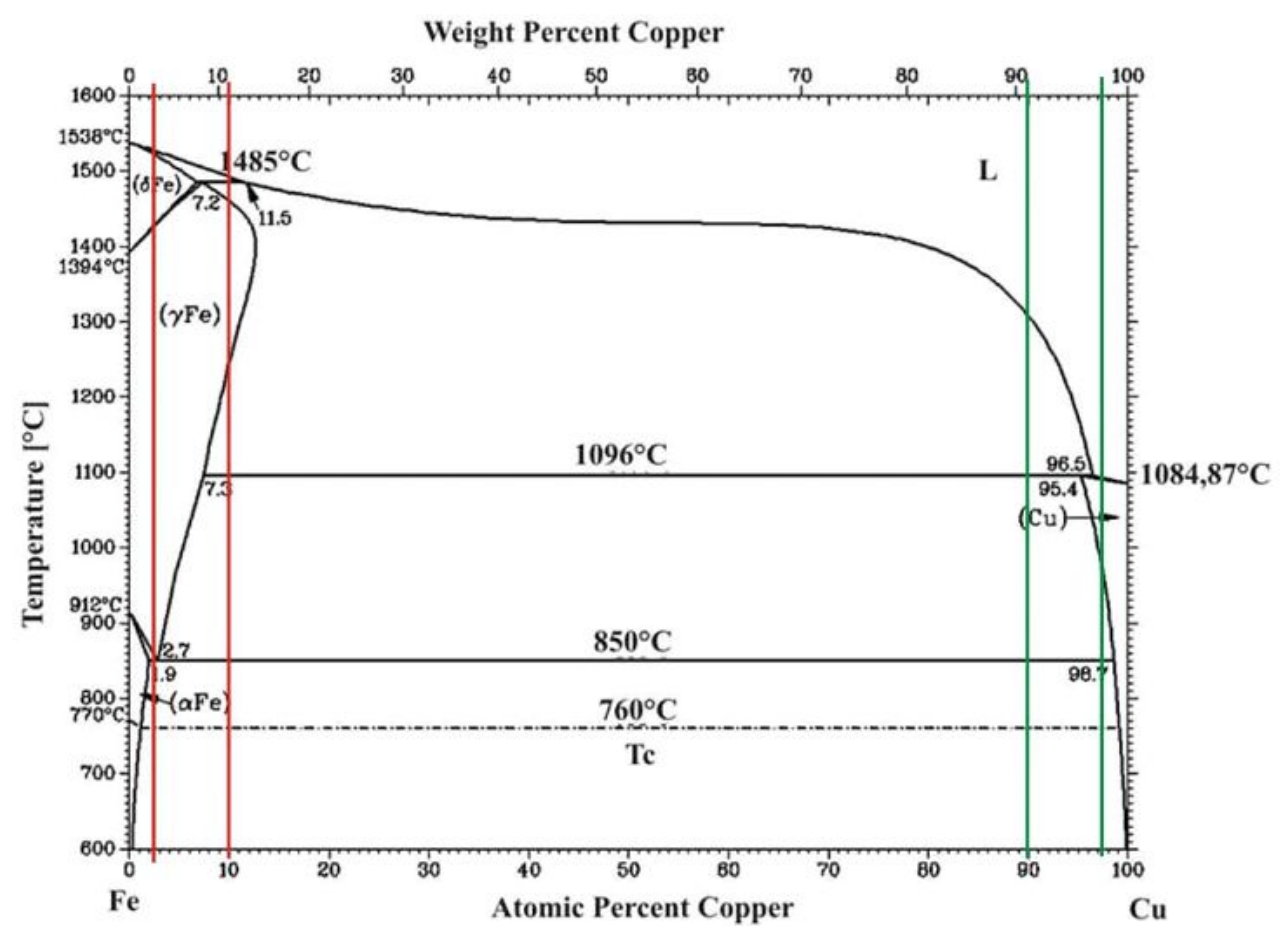

Figure 2.1: Phase diagram for the Fe-Cu-system [Massalski90], green lines indicate the region investigated within this work, red lines the alloy range of the associated Diploma thesis

According to Benjamin's treatise on mechanical alloying [Benjamin90], the alloying of a brittle and a ductile element requires a minimum solubility, to solve the brittle particles. Although this requirement is not fulfilled it has been shown before, that $\mathrm{Fe}$ and $\mathrm{Cu}$ can be alloyed to a substantial amount far outside side boundaries given by the equilibrium phase diagram.

For both sides of the composition range the lattice parameter (also given as nearest neighbour distance or average atomic volume) increases with increasing fraction of the minority component [Ma93],[Eckert93a],[Hong98]. According to Vegard's law the lattice expansion should only occur in the Fe rich alloys, as the metallic radius of $\mathrm{Cu}$ is slightly larger then the one of Fe, see above.

Figure 2.2 shows a compilation of the phase boundaries for $\mathrm{Fe}-\mathrm{Cu}$ alloys processed by different methods [Hong98]. It can bee seen that sputtering and ball milling yield the smallest two phase range.

The concentrations investigated within the scope of this work range from 2.5 at.\% to 10 at.\% $\mathrm{Cu}$, indicated by red vertical lines in Fig. 2.1. Within the same binational project [Deutsche Forschungsgemeinschaft: Germany (University of Göttingen)/ South Korea (University of Ulsan) cooperation under the contract KI-230/33-1], the analysis of Cu-rich material with 2.5 at.\% to 10 at.\% Fe was conducted by Schmidt within an associated diploma thesis 


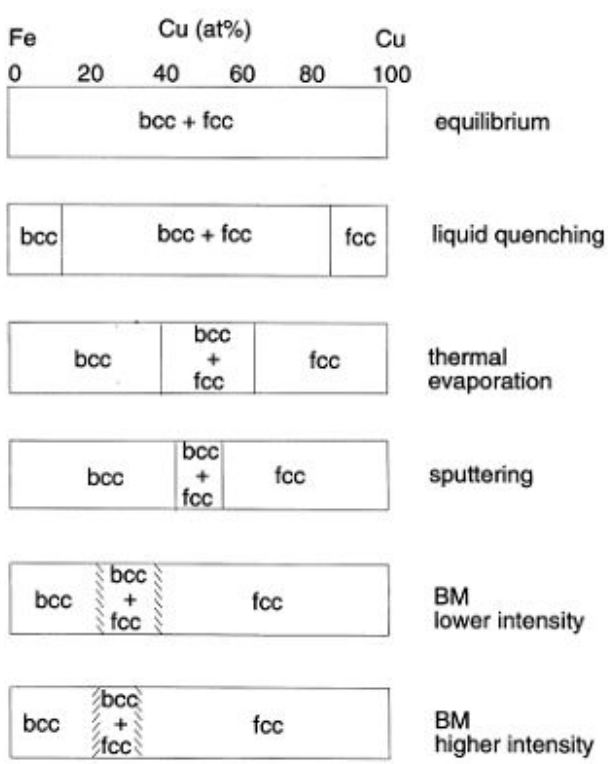

Figure 2.2: Phase boundaries for Fe-Cu alloys processed by different methods [Hong98]

[Schmidt07].The alloy range of the associated Diploma thesis is indicated by green vertical lines in Fig. 2.1. Comparing these concentrations with the phase boundaries given in Fig. 2.1 , it can be seen, that the $\mathrm{Cu}$-rich material can be expected to be single phase fcc, while the Fe-rich material can be anticipated to be single phase bcc.

\subsection{Ball milling}

Synthesis of nanocrystalline materials has been achieved by many different methods. These methods can be differentiated into the class of consolidation of small clusters and the class of breaking down bulk material into smaller dimensions. The first attempt to produce nanocrystalline powder particles was archieved by Gleiter [Gleiter89] by means of the inert gas condensation method. Since those first approaches several other methods evolved like mechanical alloying, spray conversion processing, severe plastic deformation, electrodeposition, rapid solidification from the melt, physical vapor deposition, chemical vapor processing, co-precipitation, sol-gel processing, sliding wear, spark erosion, plasma processing, autoignition, laser ablation, hydrothermal pyrolysis, thermophoretic forced flux system, quenching the melt under high pressure, biological templating, sonochemical synthesis, and devitrification of amorphous phases [Suryanarayana00] .

Each of these numerous methods offers a different range of grain sizes, morphologies and 
textures depending on the process variables (type of mill, milling container, milling speed, milling time, grinding medium, ball-to-powder weight ratio, extent of filling the vial, milling atmosphere, process contrail agents and temperature of milling [Suryanarayana01]). All the process variables and their influence cannot be covered within this short treatise on ball milling. For further details it is referred to the literature [Suryanarayana01], [Koch93], and [Koch97].

One of the most interesting points in ball milling is its capability for production scale-up. Ball milling is already an industrial process and has shown its ability to provide large quantities of material. The chemical industry has been employing this process for decades for the production of high-surface nanometre sized catalyst particles. Additionally, many different systems and material classes (pure metals, intermetallics, ceramics, compounds, immiscible systems) have successfully been produced by ball milling, making it an extremely versatile processing route.

The most prominent mill types are SPEX shaker mills, planetary ball mills and attritor mills. As the powders within this work were produced in a high-energy planetary ball mill (AGO-2) the following discussion will focus on this type of mill. The name planetary ball mill stems from the planet-like movement of the vials. The vials are mounted on a rotating support disc so they can additionally rotate around their own axes either in the same sense as the support disc or contrariwise.

According to literature, mechanical alloying (MA) and mechanical milling (MM) describe two slightly different processes conducted in the same apparatus. While in mechanical alloying blended mixtures of elemental powder are subjected to the grinding in a ball mill, in mechanical milling pre-alloyed powders are structurally refined, e.g. yielding smaller grain size or reducing the order in intermetallics. Thus, for mechanical alloying mass transfer is necessary, while it has not to be involved in mechanical milling.

The constitutive processes in ball milling are repeated fracture and cold welding. Those two competing processes determine the resulting microstructure and alloying ability. In most ball mills the deformation is performed under a protective atmosphere. High-purity argon is the most common ambient to prevent formation of oxides or other undesirable phases as well as contamination of the powder.

Contamination of the product powder is a relevant issue, not only concerning incorporation of gas atoms but also wear debris from the balls or the vial. Especially for extended milling times and reactive materials the influence of contaminations can be significant.

Cold welding of the material can turn into a serious problem when milling ductile elements. 
The heavy plastic deformation then leads to extended cold welding and the ductility prevents fracture. However, true alloying can only occur when the competing processes of cold welding and fracture balance out. To avoid this effect, a process control agent (PCA) is often added to the initial powders. Within this study no such PCA has been used.

Generally, grain size decreases with time reaching a minimum value that is determined by the two competing processes of fracture and cold welding. In fcc metals the ultimate grain size was found to scale inversely with the melting temperature [Eckert95].

\subsubsection{Mechanism of alloying}

The repeated flattening, cold welding, fracture and rewelding series is the main constituent in the deformation and alloying process. The milled powder is either deformed during a ballpower-ball or a ball-powder-vial collision, as schematically depicted in Fig. 2.3. The high forces during impact lead to a plastic deformation of the individual powder particles, which in turn results in work hardening and fracture. The newly generated surfaces are highly reactive and enable cold welding.

When a ductile and a brittle component are milled, the particles are assumed to be soft in the early stages of deformation. The cold welding is the dominating process leading to large particles (sometimes larger then the initial powder particles) and a broad range of particle sizes. As depicted in Fig. 2.3 the particle in this stages very often have a characteristic lamellar structure. Continued impacts work harden the material and fatigue failure occurs frequently. On the one hand, fracture becomes the dominating process in the second stage of deformation. On the other hand, perpetuated impact of the balls leads to cold welding as well, resulting a refined layer structure at constant particle size.

A steady-state equilibrium between increase of particle size by welding and decrease by fracture is usually reached after a certain milling time. The smallest particles are not deformed directly. Instead, they are first welded into larger particles and then deformed. This intense mixing on the scale of the particles yields a uniform distribution of all constituents among the particles. Accumulating strain energy, the particles at this stage generally reach their saturation hardness. As the small particles are incorporated into the larger ones and the largest particles are frequently fractured, a narrow particle size distribution arises [Lee98].

This heavy deformation usually results in a multitude of lattice defects such as dislocations, vacancies, stacking faults and of course an increasing number of grain boundaries. This enhanced density of defects strongly promotes the diffusivity of solutes. Additionally, the smaller grain sizes give rise to shorter diffusion distances. In miscible systems this suffices to bring 


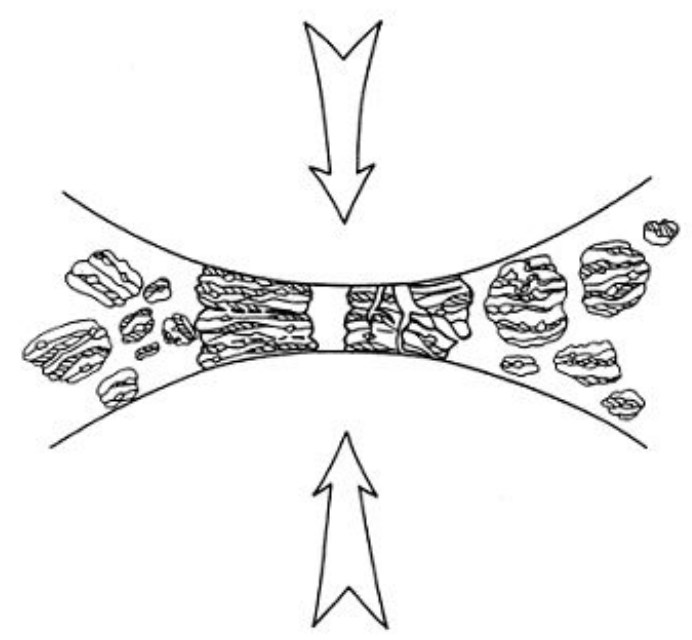

Figure 2.3: Ball-powder-ball collision of powder mixture during mechanical alloying [Suryanarayana01]

forth true alloying.

Generally the rate of refinement (in particle size, grain size, lamellar spacing, ...) depends roughly logarithmic on milling time. This rules out the starting particle size as an important parameter. Usually the grain size drops below $100 \mathrm{~nm}$ within a time of a few minutes to an hour [Suryanarayana01].

\subsubsection{Mechanisms of solid solubility extension}

Neither the actual alloying mechanisms leading to extended solutions nor the solubility limits have been thoroughly investigates for immiscible systems by now.

Although MA has been known for a long time, there is no commonly accepted model for phase formation during this process. A first description of the alloying of two ductile elements A and B with negative mixing enthalpy was given by Benjamin et. al. [Benjamin74]. Their model is based on the observation that a lamellar structure of A and B develops upon ball milling. As a consequence, the alloying process is considered to be due to an inter-diffusion process enhanced by shorter diffusion paths, an increased density of defects and an elevated temperature during ball milling.

Suryanarayana linked the formation of supersaturated solid solutions to the formation of a nanocrystalline state [Suryanarayana90]. The high density of grain boundaries is supposed to enhance diffusion and therefore solubility as the diffusion length is drastically reduced. This is supported by the finding that the solubility of $\mathrm{Mg}$ in $\mathrm{Ti}$ is raised from zero to 3at.\% when decreasing grain size from the micrometer range to the nanometre range. 
The enormous amount of grain boundary energy provided by the nanocrystalline structure is held responsible for the formation of supersaturated solution in the context of the theory of Veltl et al. [Velt191] as well. Supporting this model, Eckert et al. [Eckert93a], [Eckert93b] observed substantial alloying only after the formation of grains in the range of 10-20 nm. However, in the materials investigated in this study the grain size never drops below $20 \mathrm{~nm}$ as clearly shown by FIM, XRD and TEM. Nevertheless, atom probe investigations revealed substantial alloying on the nanometre scale even for the first milling step $(2 \mathrm{~h})$, when the grain size still ranges from 30-40 $\mathrm{nm}$ (XRD).

Within the same work by Eckert et al. [Eckert93a], [Eckert93b] an increased dislocation density is suggested as the driving force for mixing. In contrast to the observed grain size range, this part of their assumptions will be significantly supported by the TEM micrographs of the Fe-rich alloys within this work, as they will clearly show a high density of lattice defects (cf. section 4.3).

Yavari et al. [Yavari92a] suspected the large amount of oxygen in their samples, which was introduced by the ball milling process itself, to lower the enthalpy of mixing of the ternary system substantially, resulting in a negative value. As will be shown in the section on atom probe investigations, this model cannot hold here, as the oxygen is clearly inhomogeneously distributed within the $\mathrm{FeCu}$-alloy, most probably segregating at the grain or domain boundaries. Thus no homogeneous ternary mixture is formed and the thermodynamic properties are presumably not altered significantly.

An alternative process was subsequently proposed by Yavari et al. [Yavari92b] based on the finding of crystallite fragments of particularly small tip radii within the milled composite powders. For such small tip radii the capillary pressure forces are dramatically increased leading to dissolution of the fragments and further on to the formation of a homogeneous solid solution. Yavari et al. [Yavari92b] estimated the chemical interfacial energy in dependence of the crystallite diametre for the $\mathrm{Fe}-\mathrm{Cu}$ system. The calculations yielded a critical diameter of 2 $\mathrm{nm}$ for the transition from composite structure to solid solution when the capillary pressure forces the atoms at the tip of the fragments to dissolve. During continued milling repeated fracture produces ever more necking. Thus, this process could lead to full dissolution. A contribution from this process cannot be completely ruled out within this study, but the results gained by APT and FIM are not in accordance with this picture as will be shown later.

In contrast to miscible systems, MA of systems with a positive enthalpy of mixing is still far from being understood, although it has been observed for a number of alloys.

As mentioned above, a diffusion controlled mechanism as suggested for miscible alloys 
cannot hold in immiscible systems, as it should rather result in decomposition of the alloy. Currently, several models are subject of great controversy. The main topic to be addressed within these models is the mode of energy storage, when the solid solution is formed.

While Yavari et al. [Yavari92b] assumed the formation of incoherent interfaces, Gente et al. [Gente93] suggested a different model by assuming coherent interfaces for the $\mathrm{Cu}$-Co system. The chemical contribution of the interface could lead to an increase of the free energy of the composite above that of the solid solution. This could even apply to systems with a positive energy of mixing. Within this work, the early stages of milling as analysed by APT in section 4.5 will show a gradual dissolution of the respective minority component, rather supporting the assumption of coherent interfaces.

Previous to further elaboration of the detailed experimental results, a short overview of the employed experimental methods and techniques will be given in the next chapter. 


\section{Experimental Methods and Techniques}

Within this chapter the experimental methods and techniques used for the specimen preparation as well as for the characterisation will be illustrated. This includes some evaluation methods and algorithms relevant to this work.

\subsection{Dual beam Focussed Ion Beam and Scanning Electron Microscopy (FIB/SEM)}

Since the scientific interest behind this study is focussed on the physical mechanisms which play a role in the alloying processes, these mechanisms had to be separated from those mechanisms occurring during further mechanical treatment. Any encapsulation or compaction of the resulting powders was avoided when preparing samples for transmission electron microscopy and atom probe tomography.

An alternative procedure to produce the samples was developed and will be presented after a short introduction into the operating principle of the dual beam focussed ion beam and scanning electron microscopy (FIB/SEM).

\subsubsection{Operating principle}

The high resolution dual beam FIB/SEM unit used within this work cannot only gather information on the surface of a sample by imaging by either ion or electron beam. It is also able to modify and even machine the sample very precisely in the submicrometre range. The very high spatial resolution and simultaneous process control by electron imaging turn the dual beam FIB into an outstanding machining tool.

Additionally, the possibility to track the process simultaneously by electron imaging allows the site specific preparation of APT or TEM specimens. The desired region can be selected 
with higher accuracy then attainable by any other method. Therefore, these dual beam FIB systems have become essential tools for failure analysis in the semiconductor industry.

As the downside of this method, irradiation damage and ion implantation must be mentioned. This damage to the material strongly depends both on the accelerating voltage as well as on the material itself. Restricting the final processing steps to low voltages, minimises the implantation depth to about $20 \mathrm{~nm}$.

The system used within this work was a FEI nova Nanolab 600 with Ga ions of $10-30 \mathrm{kV}$ accelerating voltage. The two scanning units for an electron and an ion beam are inclined towards each other by $54^{\circ}$. The ion beam is produced by a field emission liquid metal ion (Fe-LMI) source. The ion beam interaction with the sample surface generates several kinds of signal, e.g. x-rays, secondary ions, secondary electrons, sputtered atoms and others. While xrays, electrons and ions can be utilized for imaging and characterisation purposes, the main application of sputtering is the modification of the material surface. Of course, the sputter yield (number of sputtered atoms per incident ion) differs for different materials.

Apart from imaging and ion milling, structuring of the surface can be reached by chemical vapour deposition of Pt. For this purpose a needle valve can be introduced into the chamber. At a distance of about $200 \mu \mathrm{m}$ from the surface $\left(\mathrm{CH}_{3}\right)_{3} \mathrm{Pt}\left(\mathrm{CH}_{\mathrm{p}} \mathrm{CH}_{3}\right)$ gas is inserted and the binding between metal atom and the residual molecule is cracked by electron or Ga ion beam [FEI06].

Furthermore, this instrument allows for micro-manipulation of the specimen via a needle probe. This needle (Omniprobe ${ }^{\mathrm{TM}}$ ) can freely be moved in relation the stage, offering highest comfort in transferring submicrometre sized parts.

\subsubsection{Preparation of APT and TEM samples by means of FIB}

As mentioned above the unaltered powder was to be used directly to avoid any superposition of effects of the embedding and those of the milling process itself. Thus, the powders were dusted onto double-stick carbon tape and mounted onto conventional sample holders used for scanning electron microscopy (SEM). By means of the dual-beam FIB specimens suitable for APT and TEM investigations were produced. The principle method of producing thin electron-transparent foils has been shown before,see [Giannuzzi04],[Giannuzzi99] and [Orloff02].

As scanning electron micrographs confirmed powder particle sizes in the range of $10-$ $100 \mu \mathrm{m}$, the in-situ lift-out technique described in the literature[Miller05],[Choi07b] and [Choi07a] could be applied to the samples. First the sample surface is coated with a sacrificial 
a)

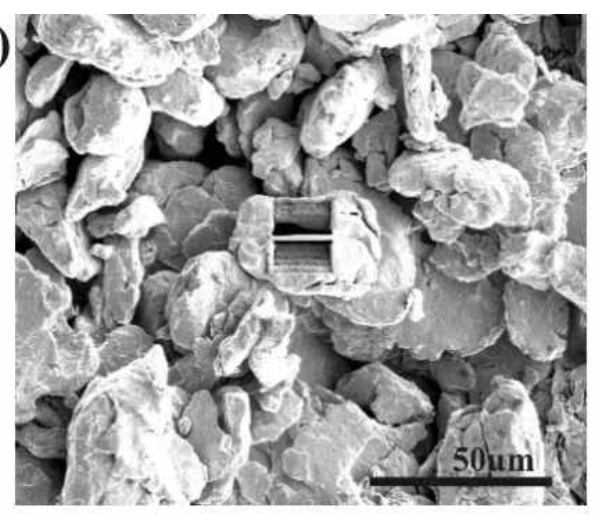

c)

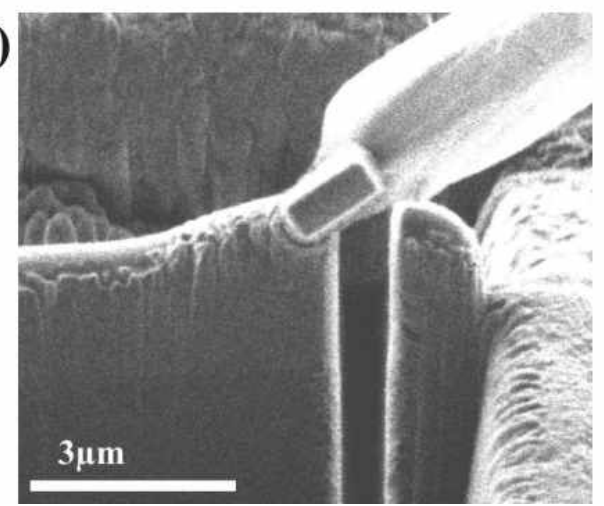

b)

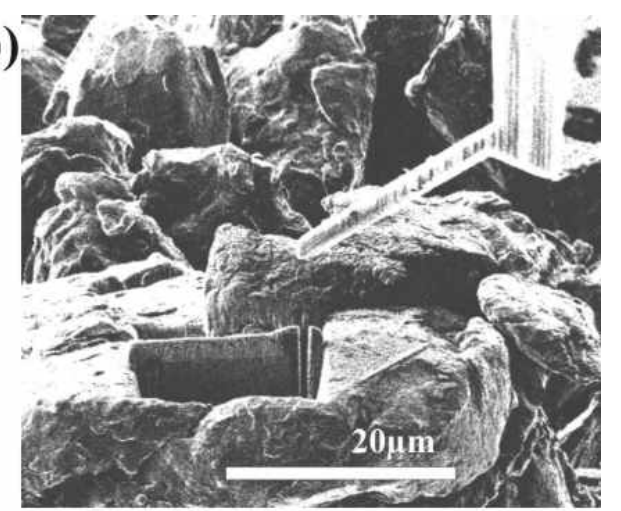

d)

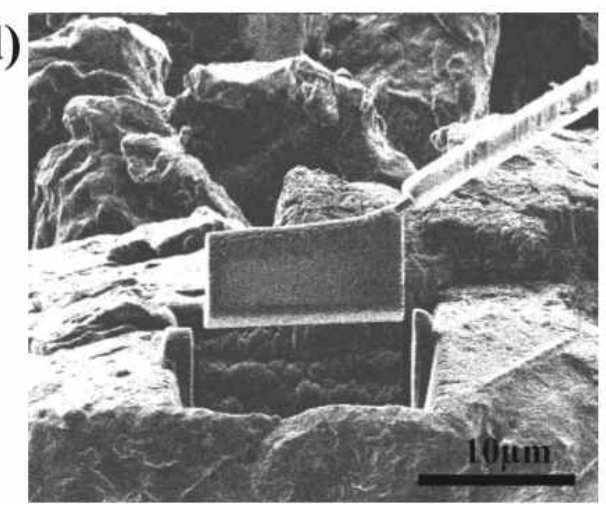

Figure 3.1: Preparation of a TEM lamella by means of FIB: a) cutting trenches, b) introducing a micrometre sized needle, c) attaching it to the lamella via Pt welding and d) lifting out the lamella

Pt-layer of $3 \mu \mathrm{m}$ thickness, to avoid severe damage by the accelerated Ga ions. Comprising of a first layer of $300 \mathrm{~nm}$ electron beam deposited Pt the following ion deposited Pt will have minimal impact on the original particle surface.

After depositing this Pt-layer, staircase-shaped cross-sections are cut on both sides beyond the layer, resulting in a lamella of approximately $20 \mu \mathrm{m} \times 10 \mu \mathrm{m} \times 2 \mu \mathrm{m}$ in size, see Fig. $3.1 \mathrm{a}$ ). By introducing a micrometre sized needle (Omniprobe) and attaching it to the lamella via Pt welding, the lamella can now be cut free and manipulated freely as shown in Fig. $3.1 \mathrm{~b}$ ) to d).

The lamella is positioned on a commercial Cu-TEM-grid and affixed to it by depositing Pt onto the lamella/holder interface. Afterwards the Omniprobe is retracted and the stage can be moved again to Pt weld the second side of the lamella/holder interface. Subsequently the lamella is thinned to electron transparency by tilting the specimen $\pm 7^{\circ}$ relative to the ion beam (Fig. 3.2).

This in-situ lift-out technique for TEM specimen preparation was further modified and adapted to produce specimens for APT investigations. Instead of lifting out the whole lamella, 
a)

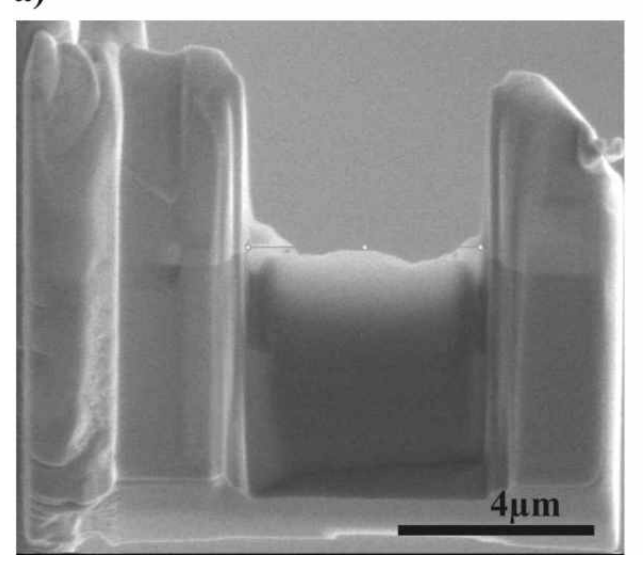

b)

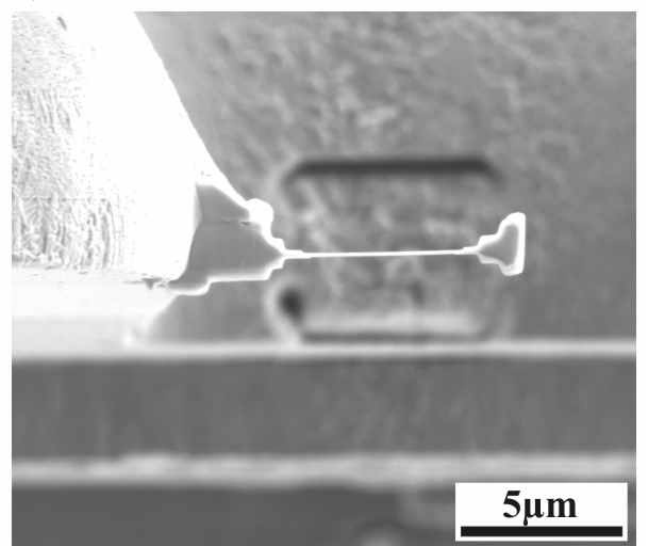

Figure 3.2: Final shape of a TEM lamella produced by FIB machining, a) front view, b) top view

it is cut into the shape of a comb (Fig. $3.3 \mathrm{a}$ ). One of the resulting cross-sectional blanks of about $2 \mu \mathrm{m} \times 2 \mu \mathrm{m} \times(5-10) \mu \mathrm{m}$ is lifted out and positioned on a $\mathrm{W}$-support. The $\mathrm{W}$-support is produced beforehand by means of standard electrochemical polishing. To yield a flat surface, the $\mathrm{W}$-support is cut to a truncated cone. Onto this platform the blank is attached by cutting a groove perpendicular to the blank/support interface and subsequently filling this groove with Pt. A second layer of Pt is deposited along the interface. This procedure is repeated for all four sides of the blank.

Tensile stresses during atom probe analysis are of the order of $10^{10} \mathrm{~Pa}$ [Grovenor87], [Miller96] and the blank/support interface runs approximately perpendicular to the direction of the tensile force, therefore, the bond between blank and $\mathrm{W}$-support has to be as strong as possible. The grooved and tongued joint enables atom probe analysis without rupture of the specimen.

A combination of side cuts and a final step of annular milling completes the preparation procedure, see Fig. 3.3 c) and d). During the initial phase the side cuts are advantageous compared to the annual milling. First of all, they remove redundant material much quicker then the annual milling. Secondly, they minimise the formation of steps and secondary tips. In addition, the implantation of $\mathrm{Ga}$ ions is almost completely avoided during the side cuts. While the first point merely optimises the preparation time, especially the second point is of prime importance to subsequent atom probe analysis. As simulations showed [Wolde-Giorgis05], sharp edges and secondary tips alter the electrical field distribution of tip dramatically. This leads to overlapping trajectories which inhibit the correct evaluation of atom probe data.

To further minimise the implantation depth of Ga ions and the resulting ion mixed zone, 
a)

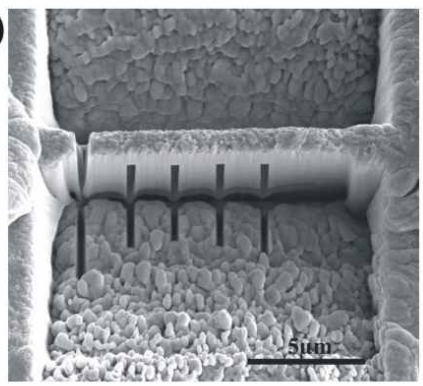

d)

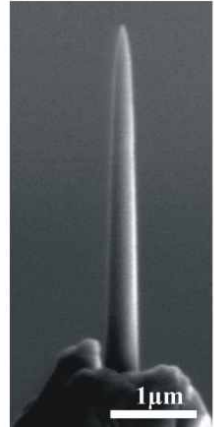

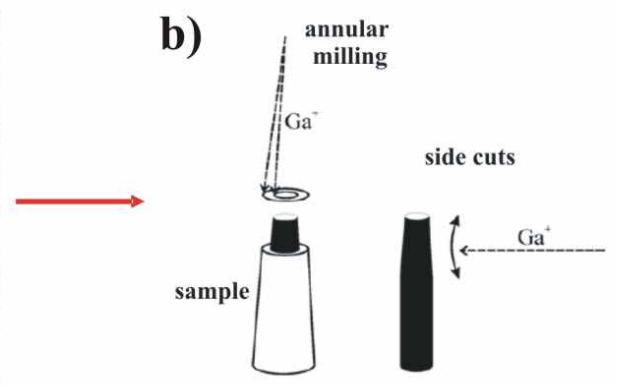

c)

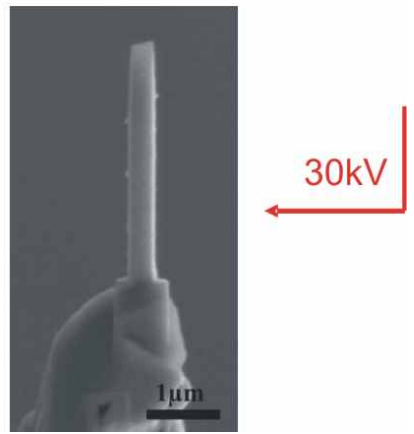

Figure 3.3: Adapting the lift-out technique: a) cutting a comb, b) placing the blank on a W-supprt, c) side cuts, d) final step: annual milling

the final step of annular milling is performed at only $10 \mathrm{kV}$. The axially-symmetric specimens of around $50 \mathrm{~nm}$ radius and very small shaft angles, which are required for APT analysis, can reproducibly be formed by this routine.

In contrast to earlier work, the yield of tips which were not prone to failure by rupture was drastically enhanced within the scope of this thesis. Employing the grooved and tongued joint for all four sides of the blank, the proportion of stable specimens was increased to $>99 \%$. Therefore, repeated sharpening of the tips was always an option to increase the measured volume in depth direction (reaching more then $1 \mu \mathrm{m}$ in depth).

\subsection{X-Ray Diffraction(XRD)}

$\mathrm{X}$-ray diffraction $(\mathrm{XRD})$ is a widespread, versatile, non-destructive and cost-effective method. Thus is it one of the most applied tools in materials science for determining the structure of a material and the phases present therein. XRD patterns not only allow the qualitative and quantitative identification of phases but also to distinguish between amorphous and crystalline materials and to determine the parameters of the crystalline structure. To obtain a diffraction pattern, the investigated distances have to be of the same order of magnitude as the wave length used. Thus, the absorber material is chosen to yield a wavelength between 
0.07 and $0.2 \mathrm{~nm}$. Detailed description of the origin of $\mathrm{x}$-rays and the different methods can be found elsewhere [Cullity67],[Warren69]. The basic formula to evaluate any x-ray traces is Bragg's law:

$$
n \cdot \lambda=2 d \cdot \sin \theta
$$

Where $n$ is the order of reflection, $\lambda$ is the wavelength used, $d$ is the interplanar spacing and $\theta$ half of the reflection angle (all angles given in Radians). This formula enables to assign interplanar spacings to the measured reflection peaks as long as the wavelength of the radiation is known. If the lattice parameter of a material is well known, uniform stress (macrostress) will be detected as a shift of the peaks. As this uniform strain expands or contracts the unit cell in an isotropic way, the interplanar spacings are changed and thus correspond with shifted peak positions compared to the unstrained crystal. The Nelson-Riley extrapolation technique (1945) permits direct precision determination of the lattice constant of cubic crystals. It ignores eccentricity and other systematic errors like absorption. The change in lattice constant $\Delta a$ over the lattice constant a depends highly linearly on the Nelson-Riley function:

$$
\frac{1}{2}\left(\frac{\cos ^{2} \theta}{\cos \theta}+\frac{\cos ^{2} \theta}{\theta}\right)
$$

$\frac{\Delta a}{a}$ is plotted versus the Nelson-Riley function and extrapolated to zero, yields the true lattice constant $a_{\text {true }}$ as the y-intercept. In contrast to the peak shift, the deviation of the diffraction pattern from delta peaks (line or peak broadening) stems as well from non-uniform strain (micro strain) as from very small grain sizes (below $\approx 0.1 \mu \mathrm{m}$ ). But, these contributions show a different theta dependence. This provides a way to separate the two effects. Both the size and the strain effect generally produce a symmetric broadening. In 1918 Scherrer was the first to analyse the contribution of small crystallite sizes to diffraction line broadening [Scherrer18]. The Scherrer Formula relates the crystallite size to the measured broadening via:

$$
\mathrm{D}=\frac{\mathrm{K} \cdot \lambda}{\beta_{\mathrm{size}} \cdot \cos \theta}
$$

Where $\mathrm{D}$ is the volume weighted crystallite size, $\mathrm{K}$ is a shape factor (mostly assumed: $\mathrm{K}=0.9$ ), $\lambda$ is the wave length of the radiation and $\beta$ is the line broadening of a reflection located at $2 \theta$ due to the effect of small crystallites. $\beta$ is given by $\beta=\left(\mathrm{B}^{1 / 4}-b^{1 / 4}\right)^{5 / 7}$, B being the Full Width at Half Maximum (FHWM) of the observed diffraction peak, and b the instrumental broadening. The instrumental broadening is the width of the peak from a specimen that exhibits no broadening beyond inherent instrumental peak broadening. 
In 1944 Stokes and Wilson first observed that the diffraction patterns of strained crystals exhibits different sort of line broadening [Stokes44]. Non-uniform strain (caused by point defects, dislocations or even extended defects) leads to systematic shifts of atoms from their ideal positions and thus results in peak broadening as follows:

$$
\epsilon_{\text {strain }}=\frac{\beta_{\text {strain }}}{4 \tan \theta}
$$

Where $\epsilon_{\text {strain }}$ is the weighted average strain of the sample. Combining these two findings, Williamson and Hall (1953) proposed a method for deconvoluting size and strain broadening, using the fact that stress-induced diffraction peak broadening follows a $\tan \theta$ function whereas crystallite size broadening has a $\frac{1}{\cos \theta}$ dependence [Williamson53].

$$
\begin{aligned}
& \left(\beta_{\text {obs }}-\beta_{\text {inst }}\right)=\frac{\lambda}{D \cdot \cos \theta}+4 \epsilon_{\text {strain }} \cdot \tan \theta \\
& \left(\beta_{\text {obs }}-\beta_{\text {inst }}\right) \cdot \cos \theta=\frac{\lambda}{D}+4 \epsilon_{\text {strain }} \cdot \sin \theta
\end{aligned}
$$

Plotting $\left(\beta_{\mathrm{obs}}-\beta_{\mathrm{inst}}\right) \cdot \cos \theta$ on the $\mathrm{y}$-axis (in radians) and $4 \sin \theta$ on the $\mathrm{x}$-axis gives the Willismson-Hall-plot. A linear fit to the data extracts the crystallite size (y-intercept) and the strain (slope).

But, in experiments the width of diffraction profiles of Bragg reflections is not always a monotonous function of the diffraction angle. Therefore, a refined version of the WilliamsonHall plot was suggested by Ungár [Ungár99b]. Based on TEM investigations, Ungár assumes the dislocations to be the main source of microstrain. Thus, the modified Williamson-Hall analysis incorporates the contrast related to dislocations by means of a contrast factor C.

Principally the contrast factor varies for the different permutations of a given set of $(h k l)$ planes. Presuming a polycrystalline untextured material or that the dislocations are uniformly distributed on the different slip systems, the contrast factors $\mathrm{C}$ can be averaged over all possible permutations of $h, k, l$. In the subsequent formulae $\mathrm{C}$ will be the averaged contrast factor. Depending on $h, k$ and $l$ it can be written as:

$$
\mathrm{C}=\mathrm{A}+\mathrm{B} \cdot \mathrm{H}^{2} \quad \text { with } \quad \mathrm{H}^{2}=\frac{\left(h^{2} k^{2}+h^{2} l^{2}+k^{2} l^{2}\right)}{\left(\left(h^{2}+k^{2}+l^{2}\right)^{2}\right)}
$$

The constants A and B strongly depend on the elastic constants of the material and the prevalent type of dislocation, edge and/or screw. Following the evaluation procedure of Williamson and Hall, the solution for dislocated crystals, the modified Williamson-Hall plot, 
can be expressed as:

$$
(\Delta \mathrm{K})^{2}=\left(\frac{0.9}{\mathrm{D}}\right)+\frac{\pi \cdot \mathrm{M}^{2} \cdot b^{2}}{2} \cdot \rho \cdot \mathrm{K}^{2}+\mathrm{O}\left(\mathrm{K}^{4} \mathrm{C}^{2}\right)
$$

where $\mathrm{K}=2 \frac{\sin \theta}{\lambda}$ and $\Delta \mathrm{K}=2 \frac{\cos \theta \cdot \beta}{\lambda}, \rho$ is the average dislocation density, $b$ is the Burgers vector and $\mathrm{M}$ is a constant depending on the outer cut-off radius of the dislocations. The exact determination of $\mathrm{M}$ can only result from a Fourier analysis of the tails of the peaks. Therefore, the simplified notation

$$
(\Delta \mathrm{K})^{2}=\left(\frac{0.9}{\mathrm{D}}\right)+\gamma \cdot \mathrm{K}^{2} \cdot \mathrm{C} \text { with } \gamma=\frac{\pi \cdot \mathrm{M}^{2} \cdot b^{2}}{2} \cdot \rho
$$

is used when it comes to practical application. Inserting eq. 3.7 into 3.9 results in:

$$
\frac{\left[(\Delta \mathrm{K})^{2}-\alpha\right]}{\mathrm{K}^{2}}=\gamma \mathrm{A}+\gamma \mathrm{BH}^{2} \quad \text { with } \alpha={\frac{0.9^{2}}{\mathrm{D}}}^{2}
$$

Instead of directly plotting $\Delta \mathrm{K}$ versus $\mathrm{K}$ as in the Williamson-Hal plot, in the modified version $\frac{\left[(\Delta \mathrm{K})^{2}-\alpha\right]}{\mathrm{K}^{2}}$ is plotted versus $\mathrm{H}^{2}$. By varying the value of $\alpha$ the standard error of the linear fit can be minimised. By means of this variation procedure, the experimentally determined values of $K$ and $\Delta K$ yield the ratio $A / B$.

Taking into account that $\mathrm{A}$ is the average value of the contrast factors corresponding to the ( $h 00$ ) reflection, $\mathrm{C}_{h 00}=\mathrm{A}$, equation 3.7 can be rewritten as:

$$
\mathrm{C}=\mathrm{C}_{h 00} \cdot\left(1-q \mathrm{H}^{2}\right) \quad \text { with } q=\frac{\mathrm{B}}{\mathrm{A}}
$$

$\frac{1}{q}$ can be directly derived from the experimental data as shown above. The practical determination of $\mathrm{C}_{h 00}$ follows [Ungár99b]. The elastic constants $c_{11}, c_{12}$ and $c_{44}$ are combined in the elastic anisotropy $\mathrm{S}=2 \cdot \frac{c_{44}}{c_{11}-c_{12}}$ and the ratio $\frac{c_{12}}{c_{44}}$. The value of $\mathrm{C}_{h 00}$ can be parametrised for different values of $\frac{c_{12}}{c_{44}}$ by

$$
\mathrm{C}_{h 00}=a \cdot\left[1-\exp \left(\frac{-\mathrm{S}}{b}\right)\right]+c \cdot \mathrm{S}+d
$$

The values of a to $d$ are given in Table 6 of [Ungár99a] for b.c.c. materials. Using these listed values for the determination of $\mathrm{C}_{h 00}$ and the experimentally derived $q$, the average contrast factor for each reflection can be given.

Finally plotting $(\Delta \mathrm{K})^{2}$ versus $\mathrm{CK}^{2}$, the linear fit provides the Grain size D in the form of the y-intercept. Further analysis of the Fourier coefficients or the detailed shape of the profiles 
leads to the dislocation density.

Within the scope of this work, the powders have been investigated both in the virgin, as received state and after embedding in epoxy resin and subsequent polishing. They will further on be referred to as virgin and polished samples, respectively. The X-ray diffraction (XRD) of the virgin powders was conducted in a Siemens D500 apparatus using a Co-source with a $\mathrm{K}_{\alpha}$ wave length of $0.17902 \mathrm{~nm}$. The measurement of the angle $2 \theta$ was performed in the range of 40 to $110^{\circ}$. The diffraction measurement of the polished samples were carried out on the X'Pert MRD diffractometer equipped as well with a Co $K_{\alpha}$ source of $\lambda=0.17902 \mathrm{~nm}$. For these samples the measurement angle $2 \theta$ could be varied from 40 to $130^{\circ}$.

\subsection{Transmission Electron Microscopy (TEM)}

Transmission electron microscopy within this project was carried out by utilising the Philips CM12 operated at $120 \mathrm{kV}$. The real space micrographs of up to 340000 -fold magnification were imaged on electron-sensitive photo plates, yielding a principle resolution of $\approx 0.5 \mathrm{~nm}$. Bright field images were employed to characterise the microstructure in dependence on the depth from the particle surface. Dark field images were consulted to give an estimate of the grain size to be compared with the grain size derived from x-ray traces. Selected Area Diffraction (SAD) was used to determine the phases present within the samples. Calibration of the diffraction constant and thereby of the camera length was performed by evaluating the diffraction rings of a single crystalline $\mathrm{Al}$ sample.

\subsection{Field lon Microscopy and Atom Probe Tomography (FIM/APT)}

The tomographic atom probe instrument equipped with a field ion microscope was the main analysis tool applied within this work. It enables imaging and chemical analysis with extremely high spatial resolution of about $0.5 \mathrm{~nm}$. In addition to this atomic resolution, the ability to detect an atom is mass independent. So the content of light elements is not underestimated as in most other techniques offering spatially resolved chemical analysis (e.g. EDX). Further on, a low noise level facilitates the detection of low impurity concentrations. Thus the atom probe tomography is a unique technique allowing 3dimensional chemical analysis on the subnanometer scale. 


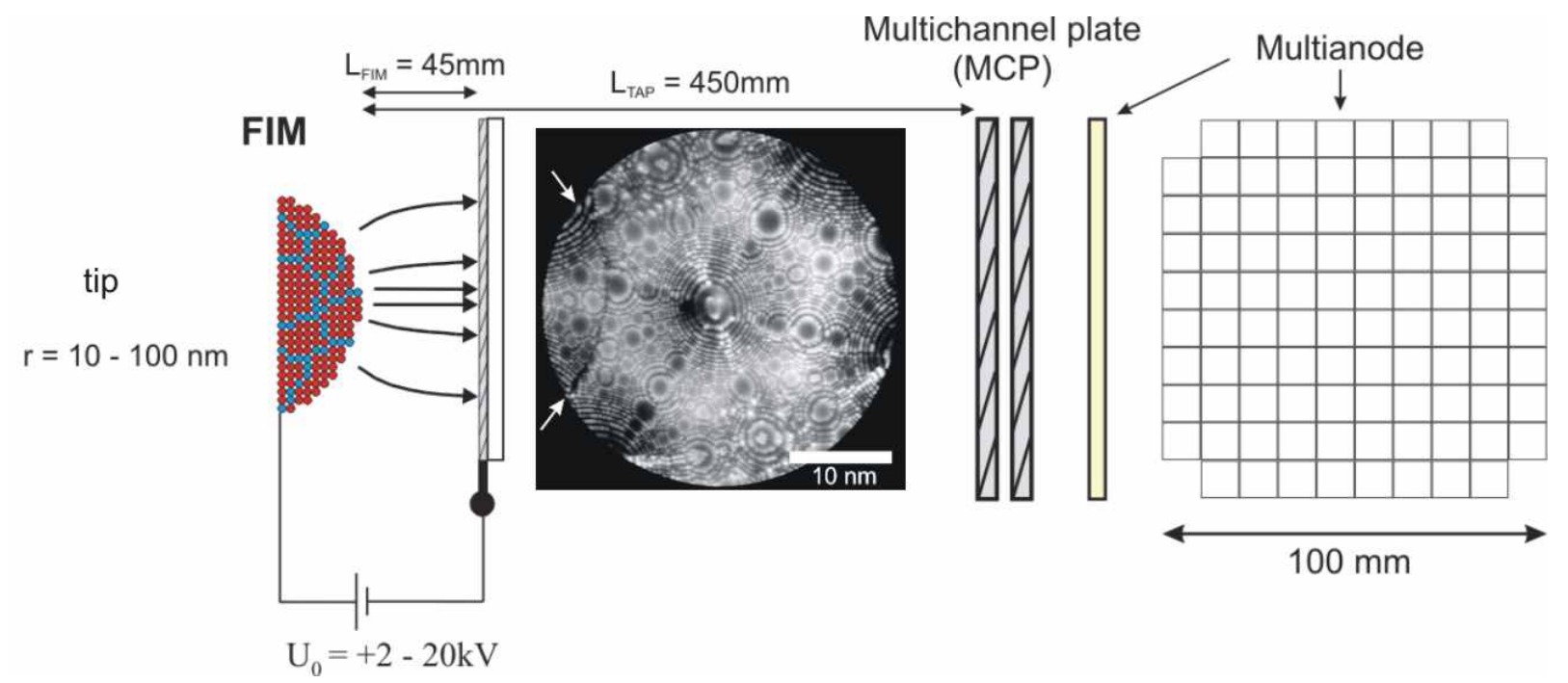

Figure 3.4: Schematic view of the Field Ion Microsope [Al-Kassab03]

Therefore, atom probe tomography has been applied systematically to characterise the homogeneity and microstructure of the mechanically alloyed Fe-Cu-powders. The local concentration, segregation effects and the distribution of impurities could be quantified on the nano-scale, depending on the different nominal compositions and processing parameters. The time-consuming sample preparation and the destructive nature of the progress clearly present the drawbacks of the method. In the following the methods of field ion microscopy and atom probe tomography will be introduced with an emphasis on the evaluation methods and algorithms relevant to this work. A more thorough treatment of the different aspects and types of tomographic atom probe can be found elsewhere [Miller96].

\subsubsection{Field Ion Microscopy (FIM)}

The fundamental requirement in Field Ion Microscopy and conventional Atom Probe Tomography is the electrical conductivity of the specimen. The tip shaped specimen with a radius of curvature of $10-100 \mathrm{~nm}$ at the apex is introduced into a UHV recipient with a residual pressure of $\approx 10^{-7} \mathrm{~Pa}$. After cooling down to temperatures $<100 \mathrm{~K}$, a positive direct current $(2-20$ $\mathrm{kV}$ ) is applied to the specimen. Opposite the specimen, at a distance of $45 \mathrm{~mm}$, a combination of two serial micro channel plates (MCP) and phosphor screen are positioned (see Fig. 3.4). The screen itself is on ground potential.

The imaging is induced by a material dependent image gas such as $\mathrm{Ne}, \mathrm{He}$ or Ar. The partial pressure of the respective image gas has to be chosen carefully. On the one hand, the supply 
of gas must be sufficient to produce a steady and bright image. On the other hand, the mean free path has to be smaller then the dimension of the recipient. Generally the gas pressure is chosen to be $\approx 10^{-3} \mathrm{~Pa}$. When applying the voltage between specimen and MCP, a strong field is produced. In this electrical field the atoms of the image gas are polarised and drift to the needle shaped tip. The field depends on the applied voltage $U$ and the radius of curvature $r$ as follows:

$$
\mathrm{F}=\frac{\mathrm{U}}{k \cdot r}
$$

The geometric factor $k=5 . .7$ depends on the exact form of the tip. It reflects the deviation from a perfectly spherical cap $(k=1)$. The polarised gas atoms possess rather high kinetic energies. Therefore, they have to be thermally accommodated by inelastic collisions with the tip surface before being field absorbed at protruding sites such as kinks and ledges (Fig. 3.5). At these protruding sites the local radius of curvature is smaller and hence the field is locally higher.

At an image gas dependent field strength and a critical distance between an absorbed gas atom and the surface $(\approx 0.5 \mathrm{~nm})$, the image gas atoms can emit an electron to the tip by quantum mechanical tunnelling process. This field ionised gas ion is repelled by the surface and attracted by the MCPs. Within the MCPs they generated electron clouds, which again impinge on the phosphor screen and are turned into bright spots. The geometrical arrangement of these spots reflects the surface topography of the specimen.

Looking at tip at the atomic scale, a hemispherical cap consists of discrete layers of atoms in different crystallographic direction. This leads to kinks and ledges. The ionisation probability is maximum at the most protruding sites. The majority of gas ions are ejected from these sites. Thus, the low index crystal planes image as concentric rings on the screen. Grain boundaries (indicated by white arrows in (Fig. 3.6) break the symmetry of the field ion micrograph and give rise to a dark contrast.

The geometrical projection of the surface onto the screen can be approximated by gnomic or a stereographic projection. Being isogonic, the latter is of advantage when indexing the poles. The average magnification reached at the apex is of the order of $10^{6}$.

When an image of the surface is obtained and the applied voltage is increased even further, atoms of the specimen itself can be desorbed. This phenomenon is called field evaporation. The specimen is eroded layer by layer. As the shaft angle of the tip is finite, the radius increases with ongoing field evaporation. The magnification decreases meanwhile. 


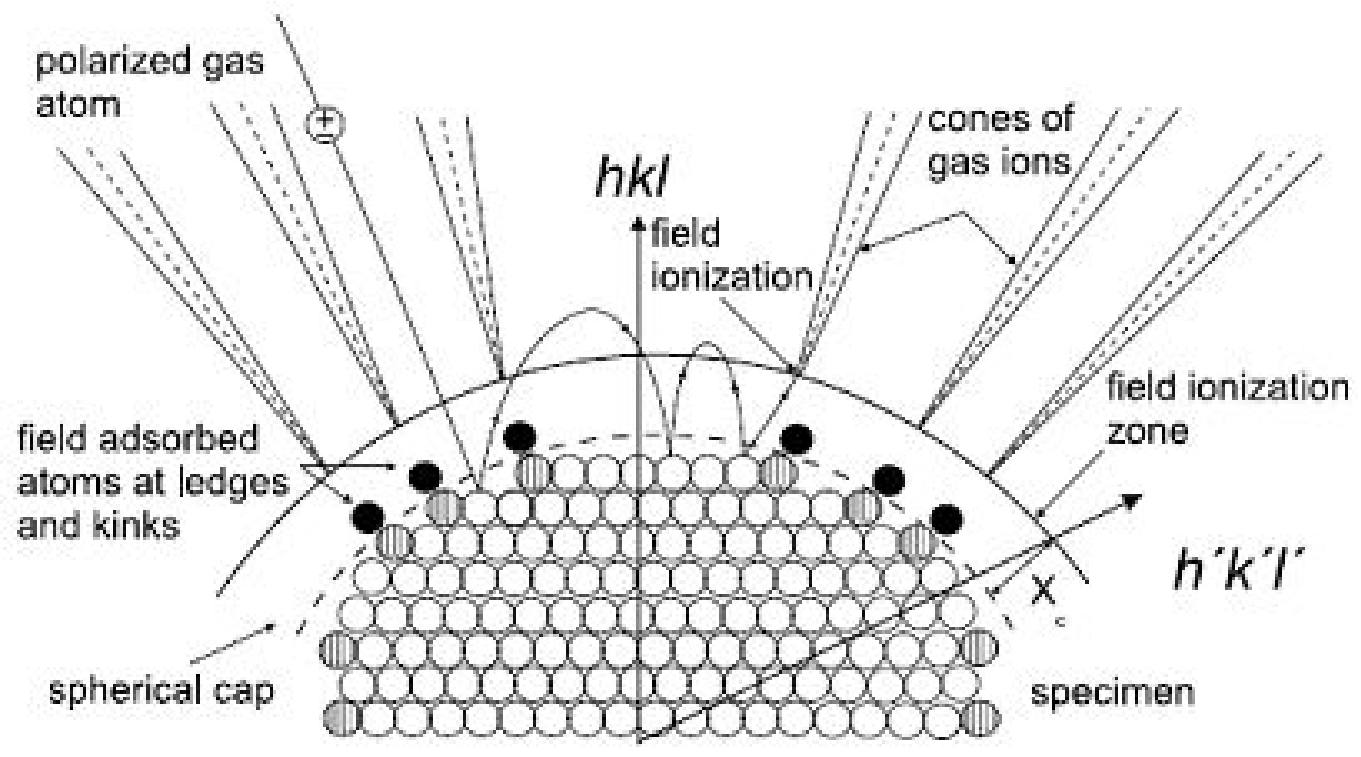

Figure 3.5: Thermal accommodation, field absorption and field ionisation at the tip apex [Miller96]

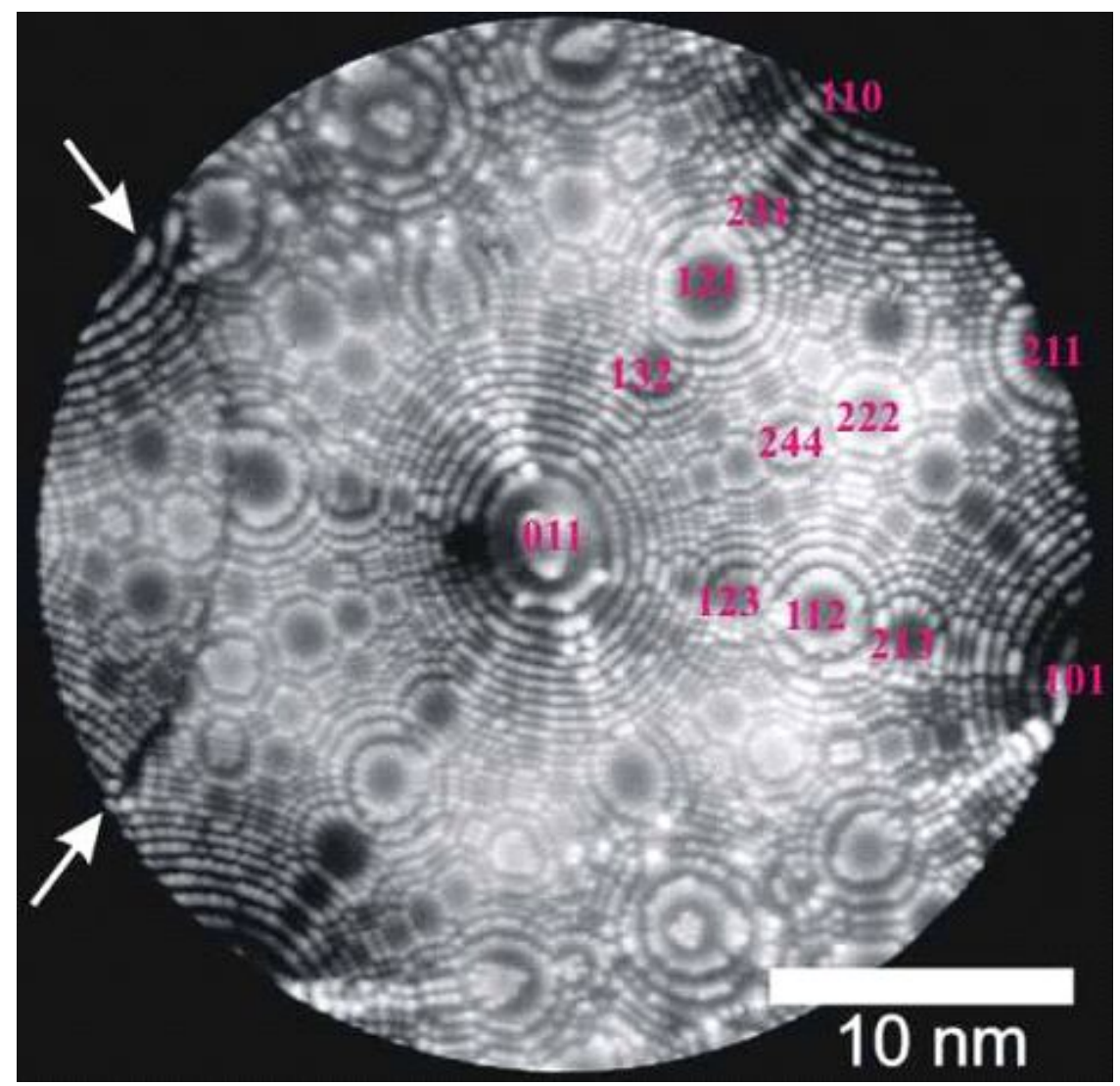

Figure 3.6: Indexed field ion micrograph of (011) oriented W, 70K and 12kV 


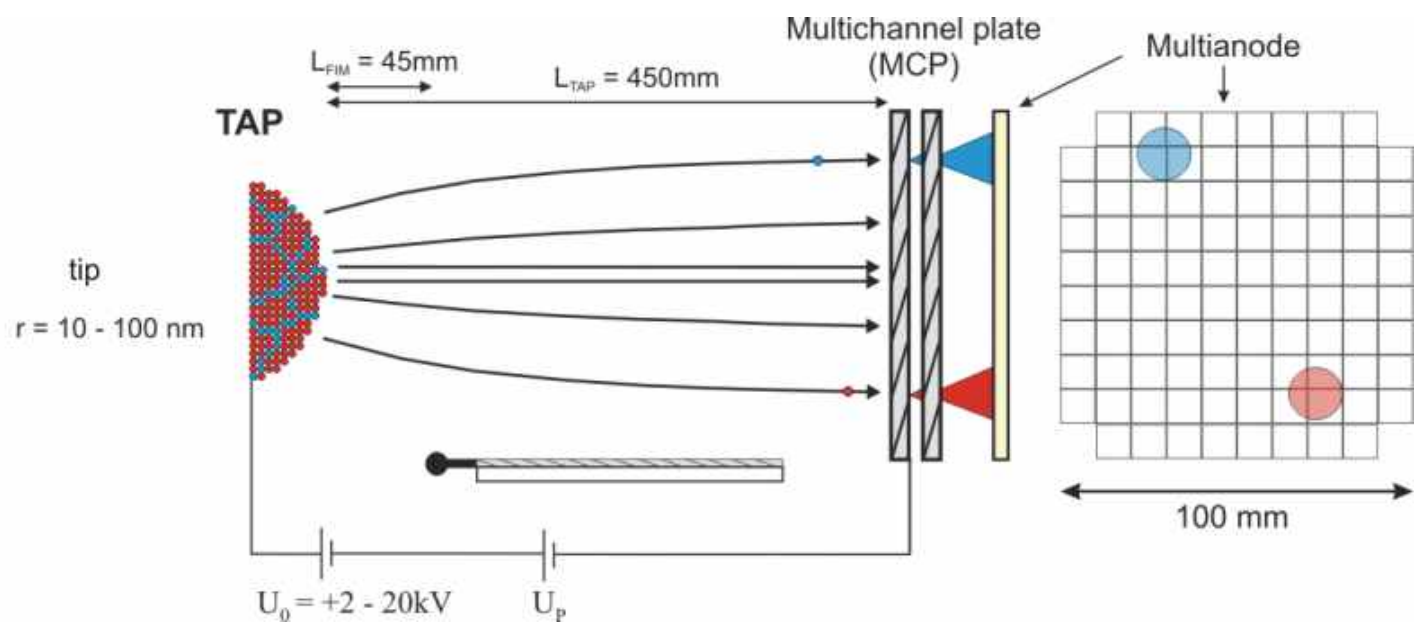

Figure 3.7: Schematic view of the Tomographic Atom Probe [AI-Kassab03]

\subsubsection{Atom Probe Tomography (APT)}

Atom probe tomography is based on the process of field evaporation, which was described above. In contrast to the FIM, during APT no image gas is present. But, the direct voltage Udc is superimposed by a pulsed voltage Up, increasing the total voltage by $10-25 \%$ within the pulse intervals of $10 \mathrm{~ns}$ with a puls frequency of $1 \mathrm{kHz}$. The direct and puls voltage are selected in such a manner to enable field evaporation only during the puls. Thus, a true measurement of the time of flight of the desorbed ions is enabled.

The imaging unit of MCPs and phosphor screen is hinged down, giving way for the ions to travel the second set of MCPs. This second set is connected to a multi-anode array ( $10 \times 10)$. The impact of the electron cloud (red and blue cones in Fig. 3.7) is registered and the centre of gravity is used to calculate the lateral position of the ion.

The sequence of the impacts gives the depth scaling. A time of flight spectrometer is included to calculate the mass to charge ratio $\frac{m}{n}$ from the time of flight $t$ and the applied voltage $\left(U_{p}+U_{d c}\right)$. Assuming that all potential energy is acquired nearly instantaneous (strong field localisation at the tip), energy preservation yield the following equation:

$$
\begin{gathered}
\mathrm{E}_{\text {pot }}=\mathrm{E}_{\mathrm{kin}} \\
n \cdot e \cdot\left(\mathrm{U}_{p}+\mathrm{U}_{d c}\right)=\frac{1}{2} \cdot m \cdot v^{2} \quad \text { where } \quad v=\frac{\mathrm{L}}{t}
\end{gathered}
$$




$$
\frac{m}{n}=2 \cdot\left(\mathrm{U}_{\mathrm{p}}+\mathrm{U}_{\mathrm{dc}}\right) \cdot e \cdot{\frac{t^{2}}{\mathrm{~L}}}^{2}
$$

where $\mathrm{L}$ is the flight length of $450 \mathrm{~mm}, v$ is the velocity and $n$ is the charge state. So, a dataset of $\mathrm{x} / \mathrm{y} / \mathrm{z}$ coordinates and the mass to charge ratio $\frac{m}{n}$ can further be evaluated. Due to the physical design of a MCP, only about $60 \%$ of the MCP surface can be used for electron multiplication. Thus the efficiency of the detector system only reaches a value of $\approx 0.6$.

\subsubsection{Measurement conditions}

To ensure statistical significance the evaluated amount of detected atoms has to be sufficient ( $>50000$ ). Therefore, the first requirement for the measurement conditions is to ensure the mechanical stability of the tips under the influence of the electric field. As mentioned above, the electric field leads to strains of about $10^{10} \mathrm{~Pa}$. Additionally, the different elements exhibit different evaporation field strengths. Preferential field evaporation of one element between the voltage pulses has to be avoided, as it results in a lower apparent concentration.

The essential control parameters are the temperature, the ratio between pulsed and direct voltage $\frac{U_{p}}{U_{\mathrm{dc}}}$ (puls ratio), the puls rate and the flux of icoming atoms (determined by the rate of voltage increase). A combination of lower temperature (30K), intermediate puls ratio $(20 \%)$, low puls rate $(1 \mathrm{kHz})$ and a high flux of $0.007-0.015$ yielded the optimum result for the Fe-rich samples.

Within the associated Diploma Thesis Schmidt [Schmidt07] varied the temperature from $20 \mathrm{~K}$ to $77 \mathrm{~K}$, the puls ratio from $16 \%$ to $25 \%$ and decreased the puls frequency from $2 \mathrm{kHz}$ to $1 \mathrm{kHz}$. Neither of the numerous parameter combinations was superior. The failure of the specimens could not systematically be avoided.

\subsubsection{Concentration depth profiles}

The significance and correlation length of concentration variations can be determined via $1 \mathrm{~d}$ concentration depth profiles. For that purpose, the area of interest is selected and marked by means of an analysis cylinder (within this work the radius was set to amount $1 \mathrm{~nm}$ ). Dividing this volume into blocks of either constant volume or of constant number of atoms, the respective concentration is computed and plotted versus depth or equivalently versus the block number.

A homogeneous distribution of a species of atoms will result in a binomially distributed local concentrations $\mathrm{c}$ with a standard deviation sigma of 


$$
\sigma=\sqrt{\frac{c_{0} \cdot\left(1-c_{0}\right)}{\mathrm{N}-1}}
$$

Where $c_{0}$ is the average concentration within the analysis cylinder and $\mathrm{N}$ is the number of atoms per block. According to binomial statistics $95.5 \%$ of all data points of a homogeneous atom distribution do not deviate more then $\pm 2 \sigma$ from the average concentration [Krengel00]. Thus, the significance of concentration variations can be evaluated.

\subsection{5 $\chi^{2}$ test}

A reliable method, for assessing whether a certain element B is homogeneously or heterogeneously distributed within the analysis volume, is the $\chi^{2}$ test. Compared to the concentration depth profile, much larger volumes can be sampled.

In the process, the measured concentration distribution of component $\mathrm{B}$ is compared to the binomial distribution with the same average B concentration. The $\chi^{2}$ parameter for a measurement

$$
\chi^{2}=\sum_{k}^{j=1} \frac{\left(\mathrm{S}_{j}-\mathrm{S} \cdot p_{j}\right)^{2}}{\mathrm{~S} \cdot p_{j}}
$$

is a measure for the deviation from the binomial distribution, which corresponds to a statistical distribution of component B. For the determination of the chi value, the reconstructed APT volume is devided into blocks of constant number of atoms. $S_{j}$ is the number of blocks with $j$ atoms of component $\mathrm{B}, \mathrm{S}$ is the total number of blocks, $k$ is the number of classes and $p_{j}$ the probability - according to binomial statistics - for a block to fall into class $j$.

The $\chi^{2}$ value is then compared to a critical value $\chi^{2}(\alpha, r)$, which is tabulated in the literature [Bronštein00], where $\alpha$ is the level of significance and $r=k-1$ is the number of degrees of freedom. Generally a level of significance of $\alpha=0.05$ is assumed. If the measured $\chi^{2}$ value is smaller then $\chi^{2}(0.05, r)$, the hypothesis is verified. That means, the deviation between measured distribution and the binomial one is small and the B-distribution can be considered homogeneous. Otherwise, if the measured $\chi^{2}$ value is larger then $\chi^{2}(0.05, r)$, the hypothesis is discarded, the B-distribution is most probably inhomogeneous.

\subsubsection{Field Ion Image Tomography (FIIT)}

As the field of view of the conventional atom probe used within this work is rather limited $\left(20 \times 20 \mathrm{~nm}^{2}\right)$, a new technique has been developed within a previous $\mathrm{PhD}$ Thesis to enhance 


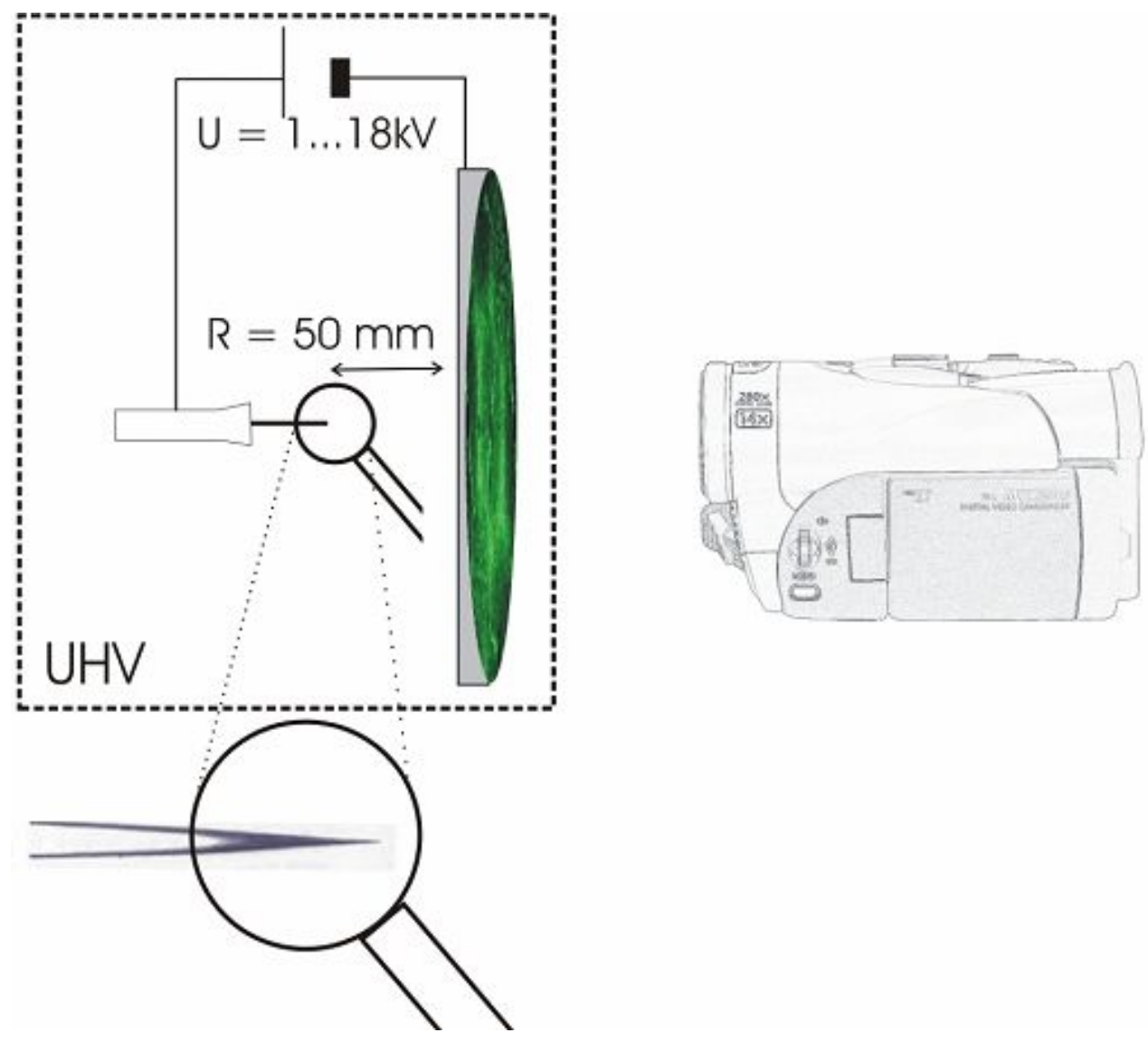

Figure 3.8: Schematic drawing of the recording of a FIIT analysis

the field of view within the possibilities of the exiting instrument.

Combining the large field of view of the FIM $\left(200 \times 200 \mathrm{~nm}^{2}\right)$ with the analysis depth of APT (several hundred nanometres), the field ion image tomography (FIIT) was developed by Heinrich et al. [Heinrich05] and Vurpillot et al. [Vurpillot04] in parallel.

For such an analysis a field evaporation sequence is recorded with a digital video camera directly from the phosphor screen, as sketched in Fig. 3.8.

Assuming a constant shaft angle of the specimen, it can be shown, that a constant rate of voltage increase $\frac{\Delta \mathrm{U}}{\Delta t}$ leads to a constant rate of material removal $\frac{\Delta z}{\Delta t}$ [Heinrich05], [Wille06a]. So, the depth scaling is given by the time scaling.

By indexing the crystallographic poles in a single field ion micrograph and determining the number of atomic layers removed per image taken an accurate depth scaling can be performed. Using commercial image processing software, $3 \mathrm{~d}$ visualising software and special algorithms developed with in the Göttingen FIM group, the 3d data obtained can be evaluated by the differences in brightness. 


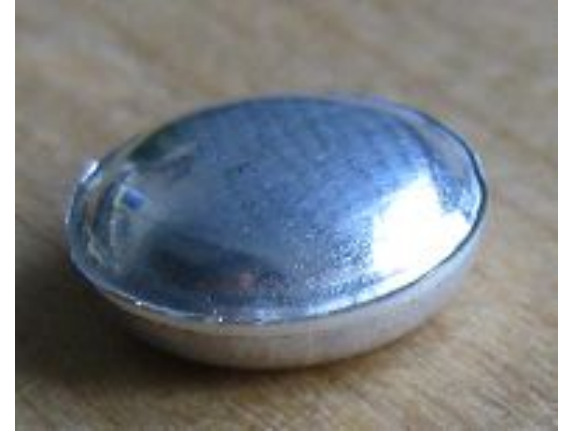

(a) bulging pans due to expansion of entrapped air

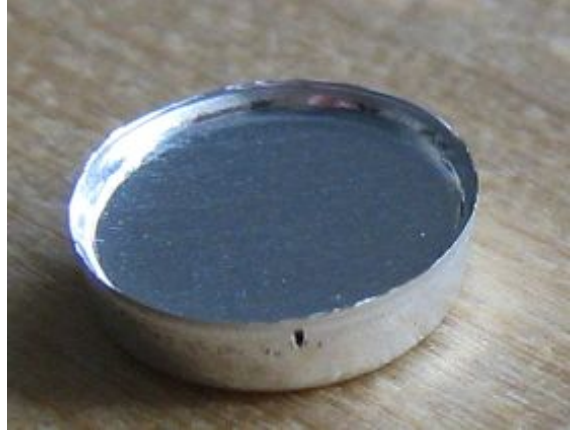

(b) perforated pans

Figure 3.9: DSC samples:comparison of unperforated and perforated pans

In brightness line profile, the brightness is plotted versus measurement distance. So, the morphology, e.g. the size and shape of precipitates, can be quantified on scale of several hundred nanometre with atomic precision. A more detailed description of the data treatment can be found in [Heinrich05], [Wille06a], [Wille06b].

\subsection{Differential Scanning Calorimetry (DSC)}

The differential scanning calorimeter (DSC) is an instrument to measure the absorbed or emitted energy during heating or cooling of specific amount of a sample. By comparing the heat flow of the sample to the heat flow of a reference material, thermodynamic measures can be determined, such as phase transformation temperatures, enthalpies of transformation and thermal transitions hysteresis data.

For a DSC measurement an exact amount of the sample is weighed in and sealed into an $\mathrm{Al}$ pan of $50 \mu$ l. Perforated pans were chosen, as the air entrapped between the powder particles lead to bulging of the pans in the first tests, see Fig. 3.9.

A second identical pan is used as a reference. Computer controlled heating ensures exactly the same heating rate for both sample and reference. During this heating process the energy Q needed to keep the heating rate constant is recorded for both heating circuits. The difference in heating power $\Delta \mathrm{P}$ between sample and reference is measured as a function of temperature $\mathrm{T}$ or rather time $\mathrm{t}$ :

$$
\Delta \mathrm{P}=\frac{d \mathrm{Q}}{d \mathrm{~T}}
$$

After conducting a second heating cycle, the trace of the second cycle is subtracted from the 
first. Thus, the reversible transformations are separated from the irreversible ones. The differential curve only displays the irreversible transformations, such as grain growth temperature, relaxation, recalecence.

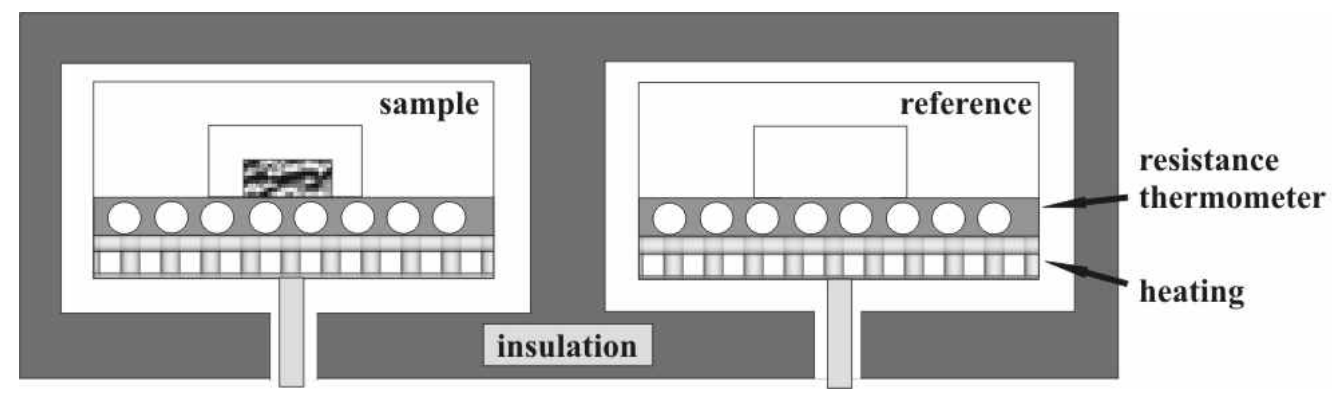

Figure 3.10: Design of a DSC consisting of two chambers with separate heating circuits

Within this work a Perkin-Elemer DSC7 instrument was used at heating rate of $10 \mathrm{~K} / \mathrm{min}$ under constant argon flow in a range between 50 and $500^{\circ} \mathrm{C}$. 


\section{Results and Discusssion}

\subsection{Morphology - light optical and scanning electron microscopy}

For the $\mathrm{Cu}$-rich alloys an extensive study of the particle shapes and size has been conducted by means of light optical (LOM) and scanning electron microscopy (SEM), as these particles were in the size range of several hundred micrometres. More detailed information on the different concentrations and milling times can be found in [Schmidt07].

According to the manufacturer's data, the initial particle size of $\mathrm{Cu}$ ranged between 44 and $149 \mu \mathrm{m}$. Even for the samples with the highest Fe-concentration of 10at.\% the particle size increases drastically with milling time. After $2 \mathrm{~h}$ it reaches $202 \mu \mathrm{m}$ and after the longest milling time of $10 \mathrm{~h}$ the particle size amounts to $835 \mu \mathrm{m}$. For the lower Fe-concentration the increase is even more pronounced, see Fig. 4.3. This increase in particle size with milling time and $\mathrm{Cu}$-contents seems reasonable, as $\mathrm{Cu}$ is a very ductile material, when processed at ambient temperature or above. As described in chapter 2, in mechanical milling the two processes of cold welding and fracture are in constant competition. The more ductile the material or the higher the volume fraction of ductile material is, the more prevails the process of cold welding.

The general trend in particle shape is the same for all concentrations: from a mixture of shapes to discs to oblate spheres resulting in spheres, see Table 5.1 in [Schmidt07].

As the inner structure of a particle may provide an indication of the mixing process, $\mathrm{Cu}$ 2.5at.\%Fe was investigated by LOM. The samples after $5 \mathrm{~h}$ milling time had the advantage of being spherical. After embedding the particle in epoxy resin, the epoxy block was subjected to grinding and subsequent polishing. After each sequence a light optical image was recorded. A series of 13 successive images is shown in Fig. 4.1.

The enormous size of the void found inside the spherical particle is noteworthy. With a diameter of $\approx 100 \mu \mathrm{m}$ the voids are as big as the initial $\mathrm{Cu}$-particles. In addition to these voids, numerous pores are present within the particle. The primary hypothesis of dense particles had to be dismissed. The cold welding of colliding particles does not seem to be as complete 


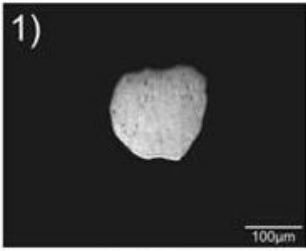

(1) $19 \mu \mathrm{m}$

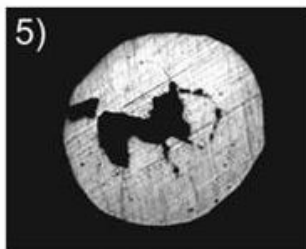

(5) $119 \mu \mathrm{m}$

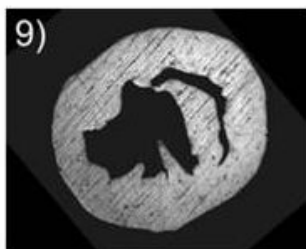

(9) $156 \mu \mathrm{m}$

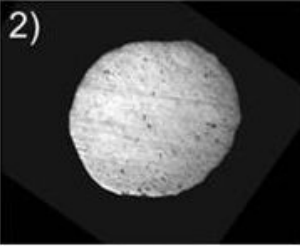

(2) $69 \mu \mathrm{m}$

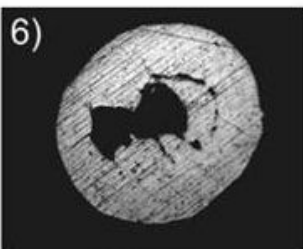

(6) $127 \mu \mathrm{m}$

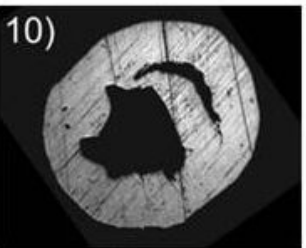

(10) $166 \mu \mathrm{m}$

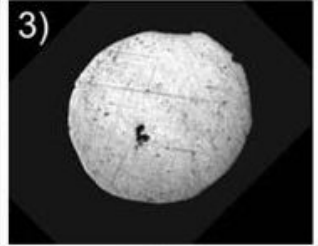

(3) $85 \mu \mathrm{m}$

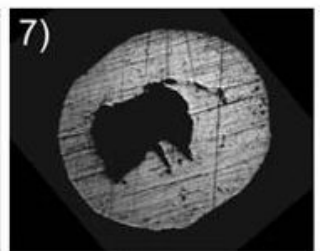

(7) $147 \mu \mathrm{m}$

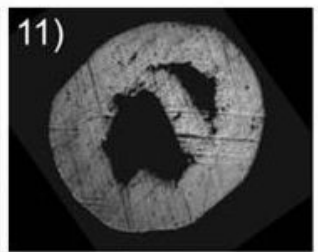

(11) $177 \mu \mathrm{m}$

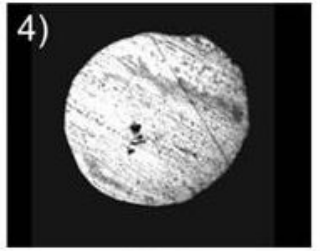

(4) $88 \mu \mathrm{m}$

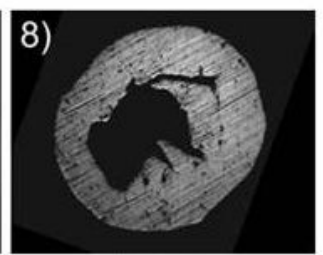

(8) $145 \mu \mathrm{m}$

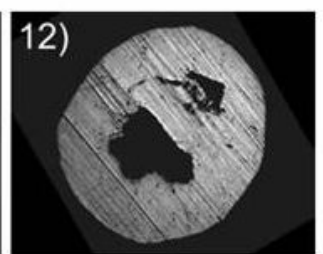

(12) $184 \mu \mathrm{m}$

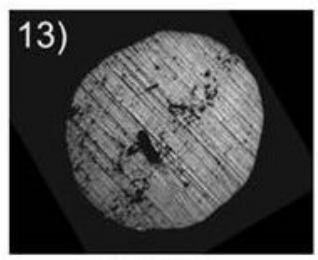

(13) $230 \mu \mathrm{m}$

Figure 4.1: Cross-sectional slices by light optical microscopy through a Cu-5at.\%Fe particle $(5 \mathrm{~h})$ after embedding and sequential polishing [Schmidt07]

as first assumed. But, most probably the subsequent milling obliterated the welding seam.

The hollow spheres seem to be stable, unless subjected to collisions with other particles. Fig. 4.2 shows mushroom shaped particles, each of which emerged from the collision of a smaller particle impinging on a bigger one. The particle in the lower part of the image illustrates the impinging in side view. The particle in the upper half exhibits a dimple in the middle of the smaller particle. This dimple most probably stems from a third particle, which was harder and thus caused the deformation without sticking to the mushroom shaped particle.

The trend of increasing particle size with milling time indicates that the competing processes of cold welding and fracture never reached equilibrium for the $\mathrm{Cu}$-rich samples within the investigated milling times. This means that inside the particle the progress in alloying and the resulting microstructure may differ strongly from the one at the surface.

In contrast to these findings, the particle size of the Fe-rich alloys ranges between 1 and 

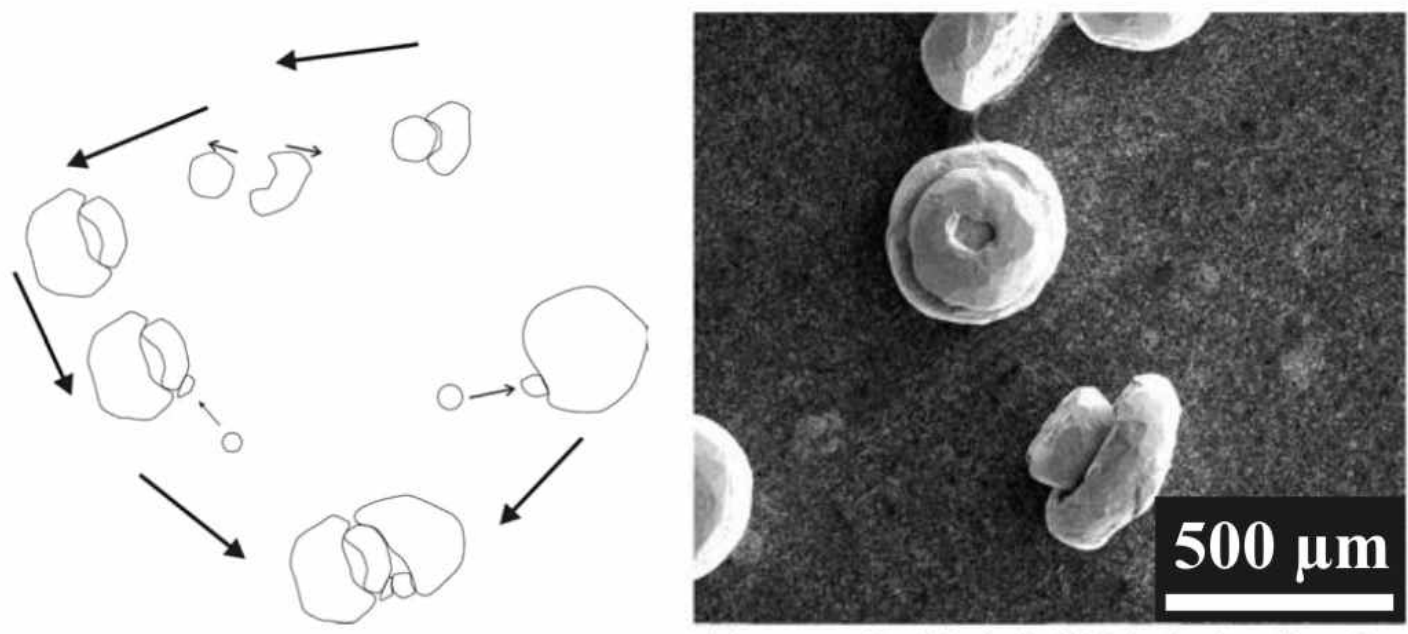

Figure 4.2: Proposed alloying mechanism and resulting mushroom shaped powder particles of Cu5at.\%Fe after $5 \mathrm{~h}$ milling time (SEM) [Schmidt07]

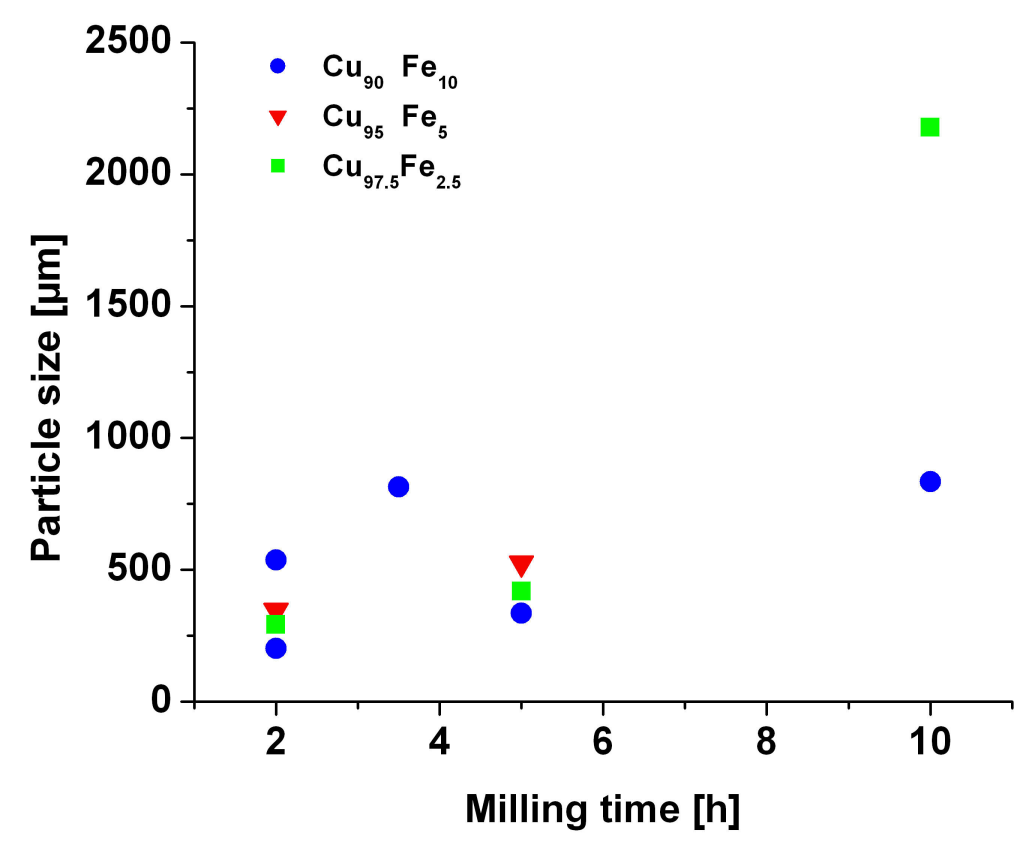

Figure 4.3: Particle size versus milling time for all investigated compositions; Cu-rich alloys show significant increase in particle size with milling time [Schmidt07] 


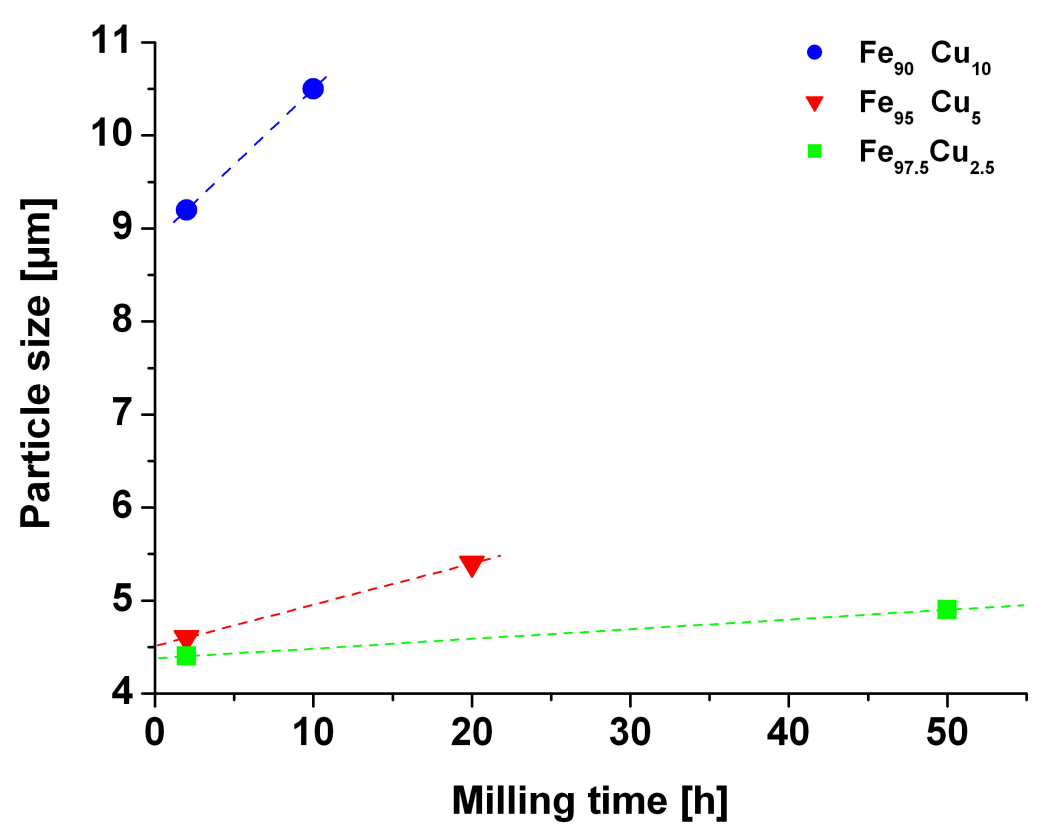

Figure 4.4: Particle size versus milling time for all investigated compositions Fe-rich alloys show rather constant particle size

$100 \mu \mathrm{m}$ with average particle sizes between 4 and $11 \mu \mathrm{m}$. Light optical micrographs were evaluated for particle sizes. As can be seen in Figs. 4.4 and 4.5 the particle size increases with increasing $\mathrm{Cu}$-contents for all Fe-rich alloys. For a fixed composition the particle size does not vary significantly with milling time. The biggest increase was found for Fe-10at.\%Cu. Raising the milling time from $2 \mathrm{~h}$ to $10 \mathrm{~h}$ resulted in an increase in particle size of $14 \%$. But, this variation is still within the limits of measuring accuracy and pales in comparison to the increases of 51 to $643 \%$ in the Cu-rich alloys, see Fig.4.3.

The stability in grain size for the Fe-rich particles indicates that - in contrast to the $\mathrm{Cu}$ rich alloys - equilibrium is reached between the two competing processes of cold welding a fracture. Cold welding not being the dominant process, the particles fracture perpetually and mixing of the material can happen on the particle size scale. Thus, the Fe-rich alloys can be expected to exhibit a rather homogenous microstructure compared to the $\mathrm{Cu}$-rich ones.

Comparing the microstructure of the embedded and polished $\mathrm{Fe}$ - and the $\mathrm{Cu}$-rich alloys (Figs. 4.5 and 4.1, respectively) the difference in porosity immediately becomes evident. The Fe-rich compounds exhibit no pores or voids, but cracks are very frequently found. While the particles size increases with increasing $\mathrm{Cu}$-content, the occurrence of cracks decreases with increasing Cu-content. This development can be followed in the polished sections of the three 
a)

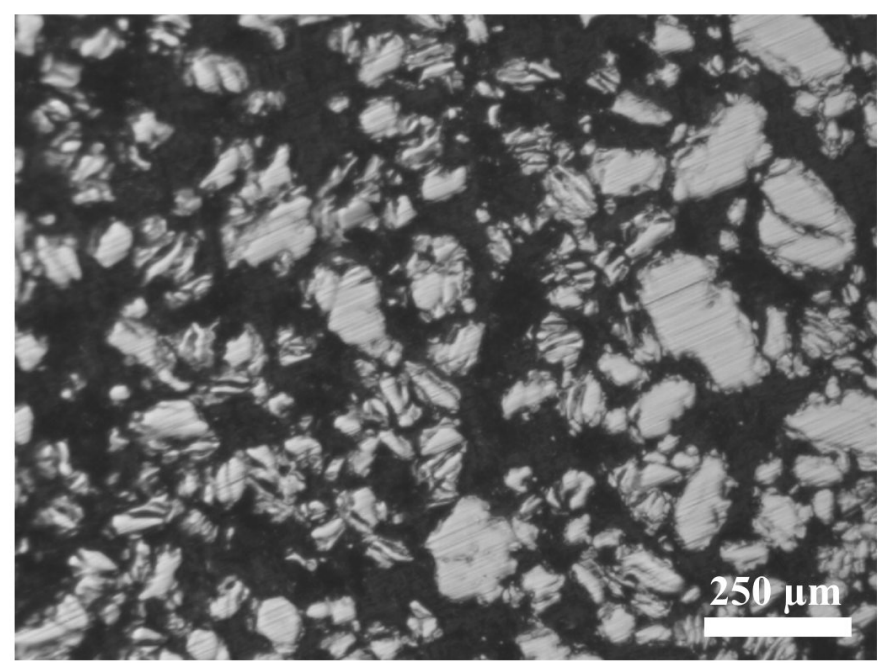

b)

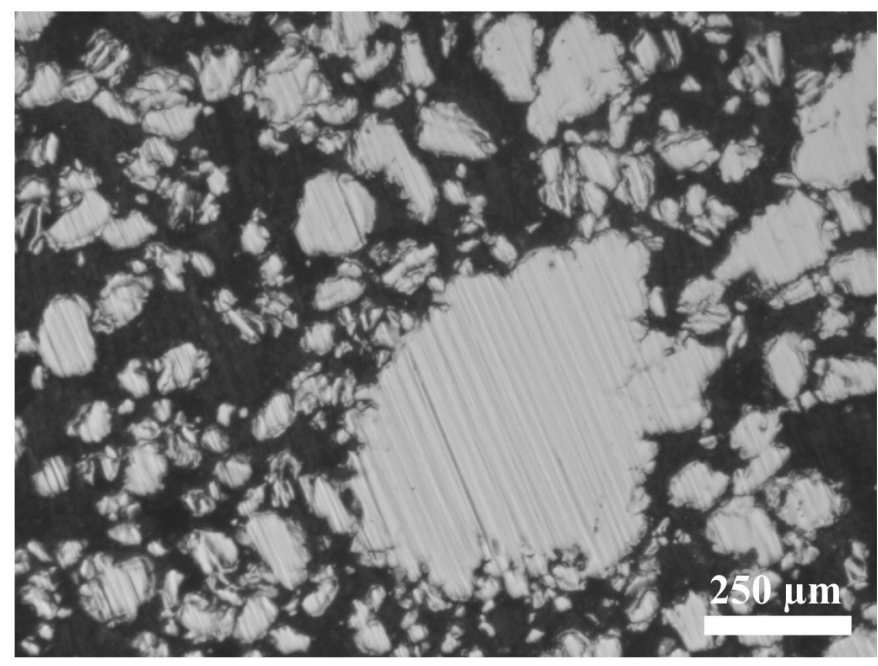

c)

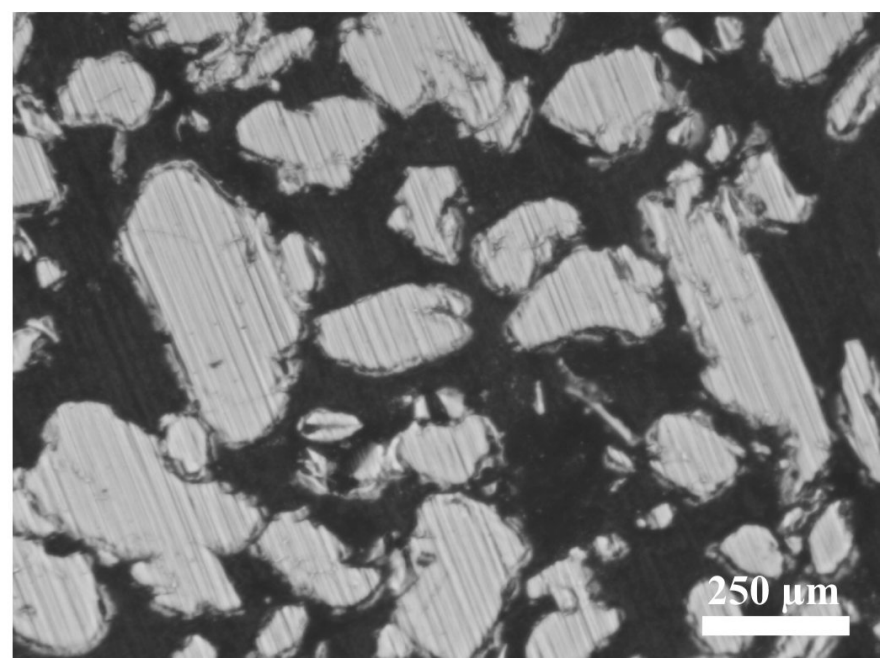

Figure 4.5: Light optical micrographs after embedding and sequential polishing: a) Fe-2.5at.\%Cu (50 h), b) $\mathrm{Fe}-5 \mathrm{at} . \% \mathrm{Cu}(20 \mathrm{~h})$ and c) Fe-10at.\%Cu (10 h) 
a)

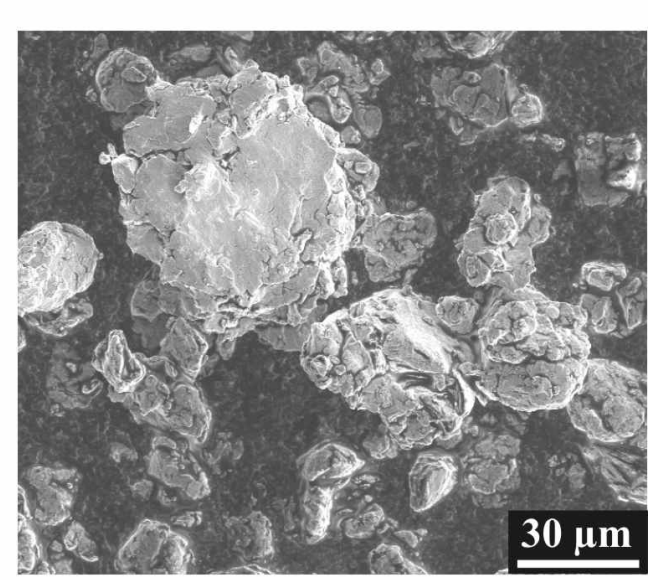

b)

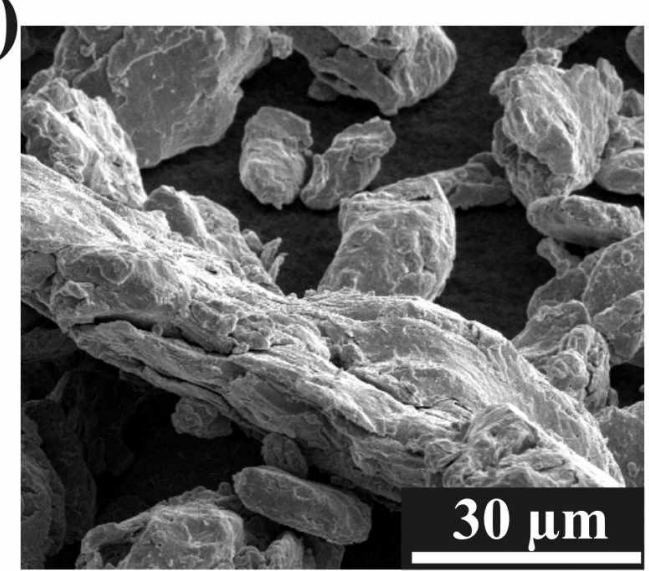

c)

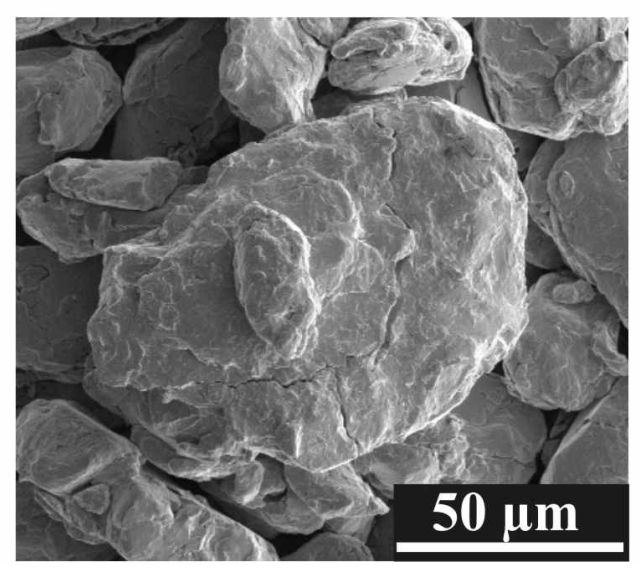

d)

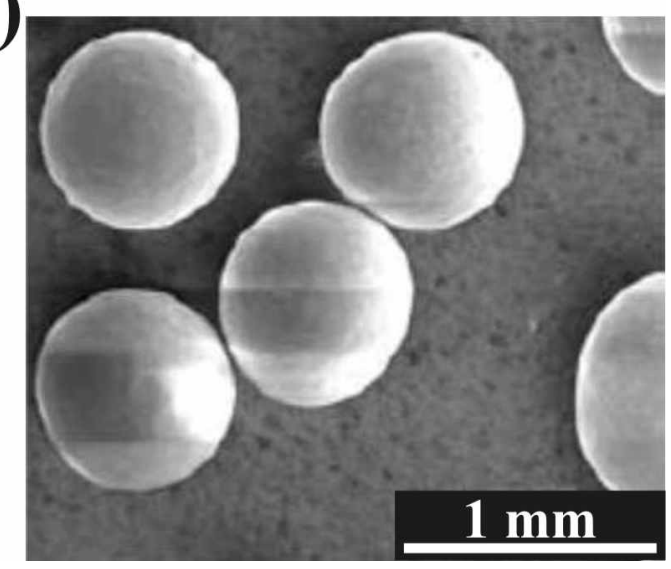

Figure 4.6: SEM images of pristine powders of: a) Fe-2.5at.\%Cu (2 h), b) Fe-5at.\%Cu (10 h), c) Fe10at.\%Cu (10 h) and d) Cu-10at.\%Fe (10 h)

Fe-rich compounds in Fig. 4.5. Both effects can be explained by the ductility of Cu mentioned above.

Not only the porosity and particle size differ strongly, but also the overall particle shape. While the shape of the $\mathrm{Cu}$-rich powder particles evolves with time from a mixture of shapes to discs to oblate spheres resulting in spheres, the Fe-rich powder particles are flaky for all states. The brittle and rough Fe-rich flakes in Fig. 4.6 a) - c) contrast heavily with the soft and smooth $\mathrm{Cu}$-rich spheres in part $\mathrm{d}$ ). With the naked eye the colour of the $\mathrm{Cu}$-rich samples ranges from golden to reddish copper. All samples shine and nearly look polished. In contrast, all Fe-rich samples exhibit rather the same greyish colour and most of them are matt.

The microstructural investigations were continued by means of XRD and TEM to gain inside into the mechanisms leading to and the alloying process resulting from these different particle sizes and shapes. 


\subsection{Diffraction study by X-Rays}

After investigating the morphology of the powder particles, the phases present were determined by means of $x$-ray diffractometry (XRD). In the first series of $x$-ray experiments using the D500 powder diffractometer, the virgin samples were measured without compaction or embedding.

Fig. 4.7 shows the $\mathrm{x}$-ray patterns for $\mathrm{Cu}-10$ at.\% Fe after various milling times, representative for all $\mathrm{Cu}$-rich alloys. The highest Fe concentration was chosen for display, due to the detection limit inherent in this method. At least 2 vol.\% of a constituent phase are needed to reliably produce a peak. Thus, the probability of detecting Fe-peaks is much higher in $\mathrm{Cu}-10$ at.\% Fe than in $\mathrm{Cu}-2.5$ at.\% Fe.

After the shortest milling time of $2 \mathrm{~h}$, the (110) peak of bcc-Fe can be detected in the $\mathrm{x}$-ray pattern of the alloy with the highest Fe contents (10 at.\%), see Fig. 4.7. For longer milling times or lower Fe-concentrations no further traces of Fe-peaks could be found.

Using Scherrer's formula the average grain size was determined from the XRD-peak broadening (cf. Fig. 4.8). Within the precision of the measurement, the grain size derived by this method stays constant over the whole range of milling times. Even after the shortest milling time ( $2 \mathrm{~h}$ ) both alloys ( $\mathrm{Cu}-2.5$ at.\% $\mathrm{Fe}$ and $\mathrm{Cu}-10$ at.\% $\mathrm{Fe}$ ) can be called nanocrystalline. The average grain size amounts to 15 and $17 \mathrm{~nm}$, respectively.

Even for the highest $\mathrm{Cu}$-contents of 10 at.\% $\mathrm{Cu}$, the shortest milling time of $2 \mathrm{~h}$ suffices to distribute enough $\mathrm{Cu}$ within the Fe-matrix to extinguish the fcc Cu-peaks. Only peaks of pure bcc Fe can be identified when compared to the tabulated powder standards, see Fig. 4.9. With regard to the detection limit inherent in this method, it can be assumed that the fraction of remaining pure $\mathrm{Cu}$ is less than about 2 vol.\% of the whole sample. The only exception to this finding is demonstrated for the material scarped from the vessel walls after $2 \mathrm{~h}$ of milling. This XRD pattern shows distinct peaks of pure unalloyed fcc $\mathrm{Cu}$. As the material sticking to the vial walls differs strongly from the loose powder, this material will not be included in the following investigations.

In Fig. 4.10 the grain size as evaluated by means of Scherrer's formula is plotted versus the milling time. Even after only $2 \mathrm{~h}$ milling time the grain size dropped to a value of $10-15 \mathrm{~nm}$, the material can be called nanocrystalline. The initially steep slope levels out for longer times reaching a steady state after about $20 \mathrm{~h}$. Obviously, this grain size value of $\approx 8 \mathrm{~nm}$ must be the one determined by the two competitive processes of dynamic recrystallisation and creation of new grain boundaries by deformation [Suryanarayana01]. 


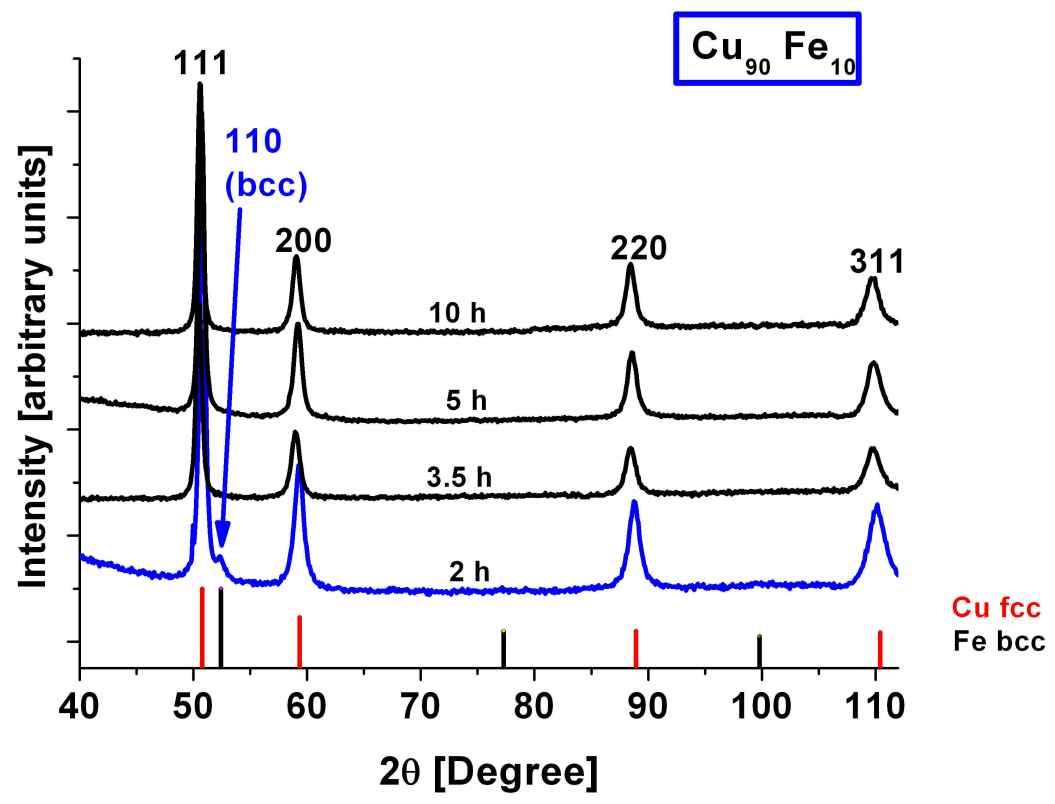

Figure 4.7: X-ray patterns of $\mathrm{Cu}-10$ at.\% Fe for various milling times, bcc Fe peaks can only be found after the shortest milling time of $2 \mathrm{~h}$ [Schmidt07]

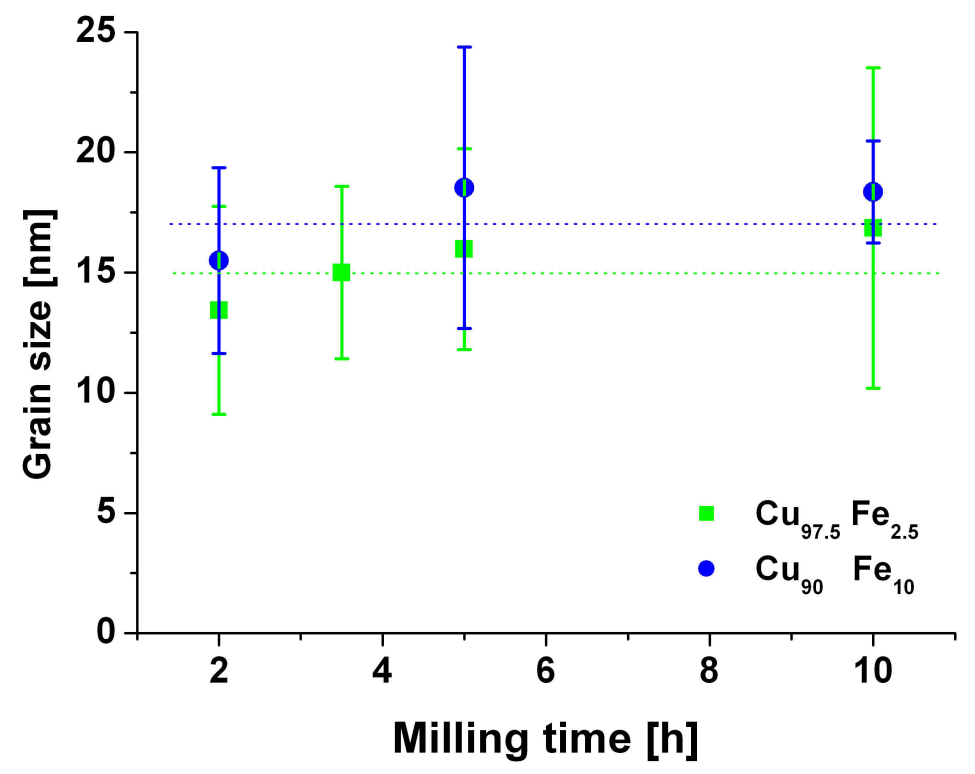

Figure 4.8: Grain size derived from the x-ray patterns employing Scherrer's formula is plotted versus milling time, grain size stays constant within error bars [Schmidt07] 


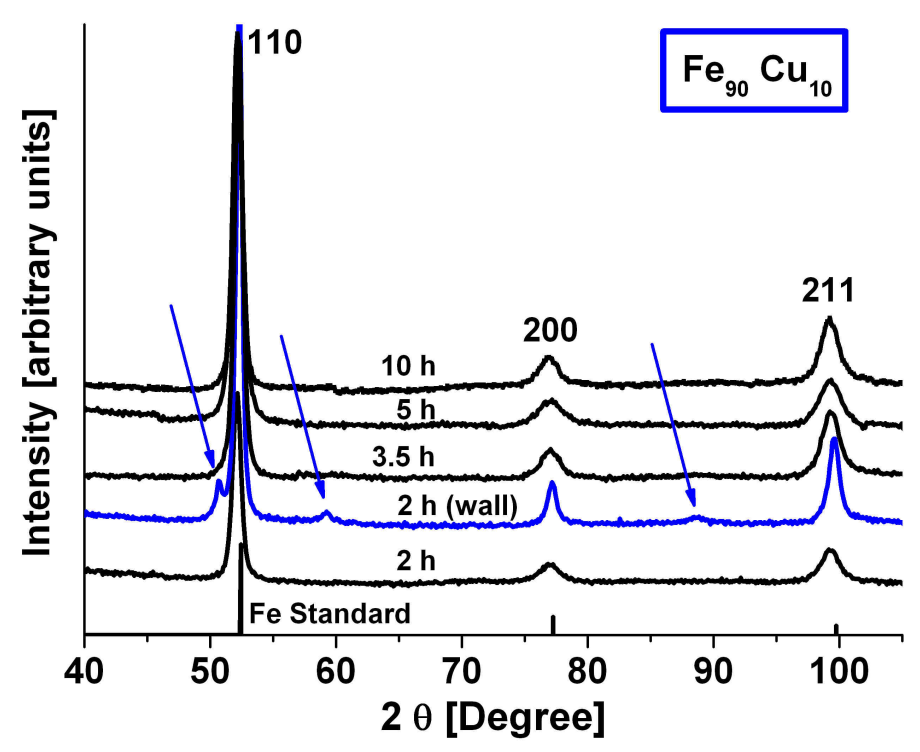

Figure 4.9: X-ray patterns of Fe-10 at.\% Cu for various milling times, bcc-fe peaks can only be detected after the shortest milling time of $2 \mathrm{~h}$ for material scraped from the vial walls

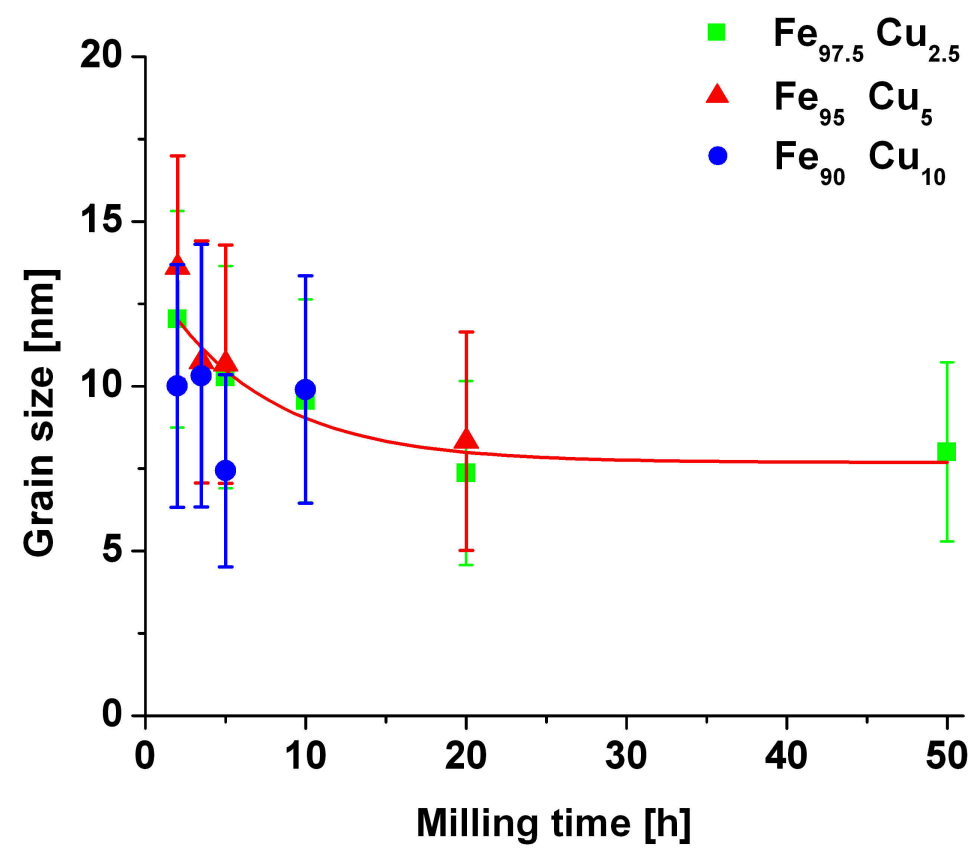

Figure 4.10: Grain size derived from the x-ray patterns employing Scherrer's formula is plotted versus milling time, steep slope levels out due to the two competitive processes of dynamic recrystallisation and creation of new grain boundaries by deformation 
This value is much smaller on the Fe-rich alloys then in the $\mathrm{Cu}$-rich alloys in agreement with literature. The melting point or the bulk modulus of the specific element determines the ultimate grain size [Eckert92].

In the second series of $\mathrm{x}$-ray experiments using the X'Pert diffractometer, the samples were embedded in epoxy raisin and subsequently polished before the measurement.

Exemplarily for these embedded and polished samples, Fig. 4.11 shows the XRD pattern for Fe-2.5 at.\% $\mathrm{Cu}$ after the shortest $(2 \mathrm{~h})$ and the longest milling time $(50 \mathrm{~h})$. Even with the naked eye a slight peak shift to the left can be seen, when comparing the dark green curve of the longest milling time with the light green one of the shortest. As described in the fundamentals (Chapter 2), a uniform stress causes this peak shift.

Following the procedure of Nelson and Riley both the virgin and the embedded and polished samples were evaluated. In the graphs the respective curves are marked 'virgin' or 'polished'.

In Figure 4.12 the lattice expansion is plotted versus milling time for all investigated Ferich alloys for both the virgin and the polished samples. The general trend is the same for all samples. The lattice expansion increases as well with increasing $\mathrm{Cu}$-concentration as with increasing milling time. For all $\mathrm{Cu}$-concentrations the lattice expansion in the polished samples is significantly higher then in the virgin ones.

The increase in lattice expansion with increasing $\mathrm{Cu}$-concentration can be understood considering the slightly larger atomic volume of $\mathrm{Cu}$ compared to Fe. The increase in lattice expansion with longer milling times can be ascribed to the progressing alloying of the larger $\mathrm{Cu}$-atoms into the Fe-matrix. So, it results as well from the different metallic radii of the two elements. But, the metallic radius of $\mathrm{Cu}$ is only less then $3 \%$ larger then the one of Fe. Taking into account the maximum concentration of 10 at.\% $\mathrm{Cu}$, the maximum lattice expansion can be expected to be $0.3 \%$. All measured values are smaller then or in the range of $0.3 \%$.

The persistently higher values for the polished samples might be caused by different region investigated. As described in the following chapter on TEM results, the morphology of the outer shell of the powder particles differs strongly from the one of the interior. Additionally, for the embedded and polished samples, the relaxed surface region is removed by grinding and polishing. Thus, only the unrelaxed inner part contributed to the XRD pattern. This results in a larger value of lattice expansion then the virgin powders, where a substantial amount of relaxed surface contributes to the XRD spectrum.

Comparing the results obtained within this work to the literature yield good agreement. As plotted in Figure 4.13, the values gained by Eckert et.al. [Eckert93b] significantly deviate 


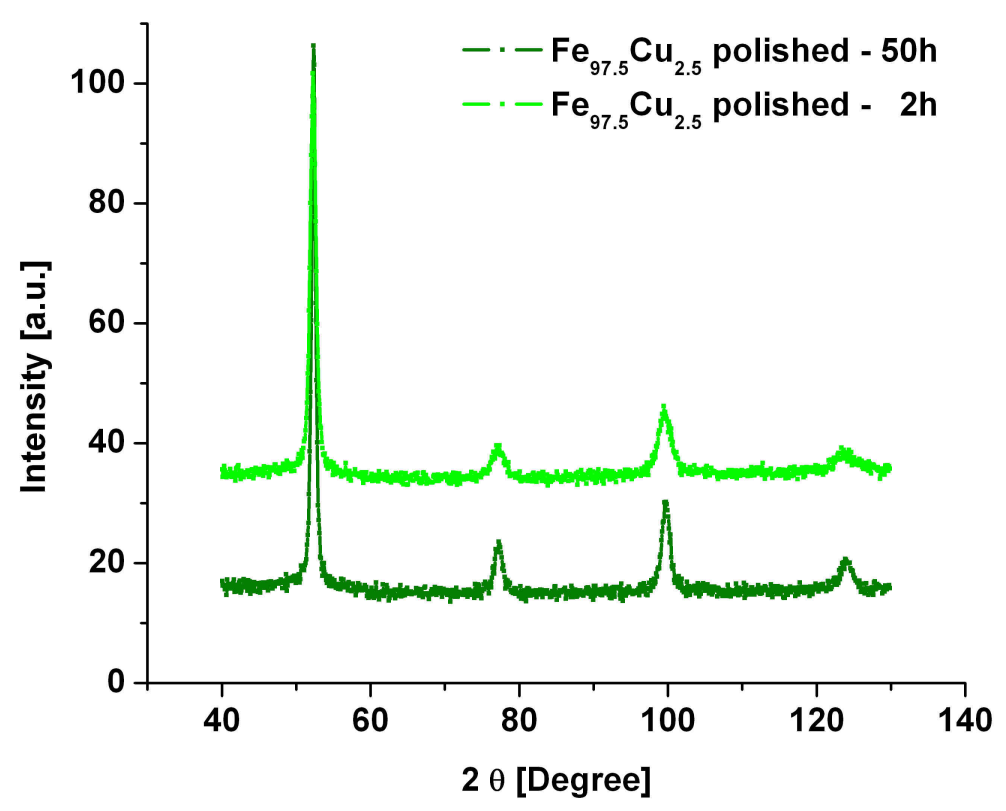

Figure 4.11: XRD patterns of Fe-2.5 at.\% Cu after the shortest (2h) and longest (50 h) milling time, exhibiting a slight shift to the left and increasing peak broadening with milling time

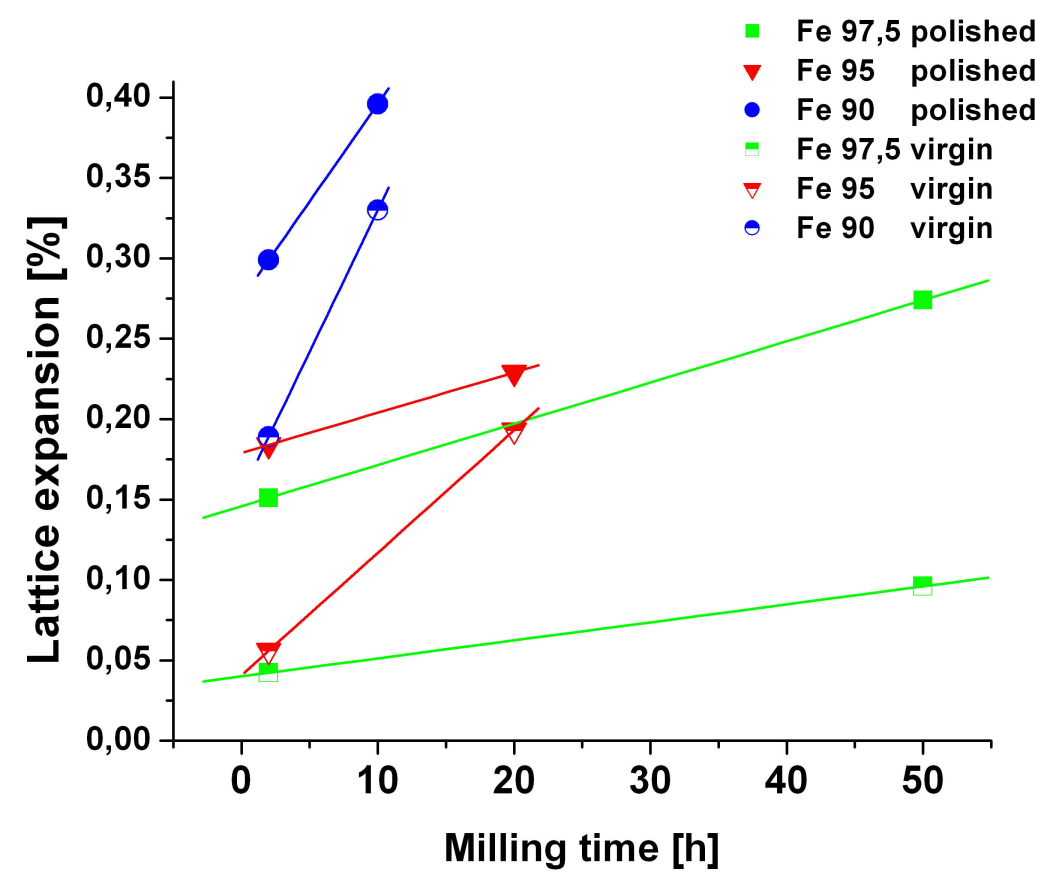

Figure 4.12: Lattice expansion in \% is plotted versus milling time in $\mathrm{h}$ for all investigated Fe-rich alloys for both the virgin and the polished samples 


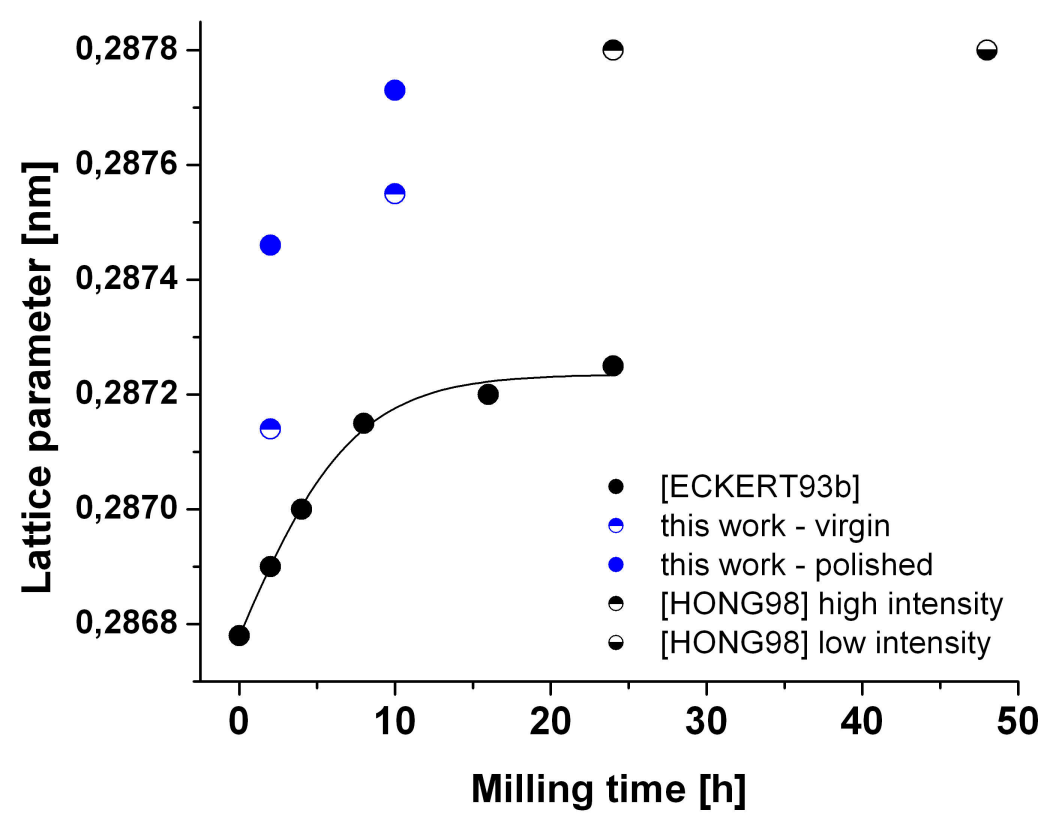

Figure 4.13: Lattice parameter plotted versus milling time for $\mathrm{Fe}-10$ at.\% $\mathrm{Cu}$, data obtained within this work compared to the literature

from the results obtained within this work. Eckert and co-workers did not use a planetary mill similar to the one utilised in this work, but a Spex 8000 shaker mill. Thus, the smaller lattice expansion might be due to the lower velocity of the impacting ball inside the shaker mill, yielding a lower degree of alloying after identical milling times. This assumption is supported by the appearance of fcc Cu-peaks in the XRD pattern of Fe-10 at.\% $\mathrm{Cu}$ after $2 \mathrm{~h}$ of milling in the Spex 8000 [Eckert93b]. In this work, the XRD pattern of Fe-10 at.\% Cu after $2 \mathrm{~h}$ of milling does not exhibit fcc $\mathrm{Cu}$-peaks, indicating a higher degree of alloying.

Furthermore, this thesis is affirmed by the study of Hong at al. [Hong98]. Employing two different milling intensities, it was shown that the same change in lattice parameter was obtained after half the milling time when the milling intensity was tripled. The AGO planetary ball mill has a higher milling intensity then the Spex shaker mill, hence giving rise to the higher lattice expansion measured within this work.

Going back to Fig. 4.11, it clearly shows an increase in peak broadening with milling time. As described in the fundamentals (Chapter 2), this results from refinement of the grain size as well as increase in microstrain. Therefore, the Williamson-Hall-method for the deconvolution of size and strain effects was applied to the measurements. Unfortunately, no corrections on the stress magnitude can be estimated, since the evaluation via Williamson-Hall-plots was 


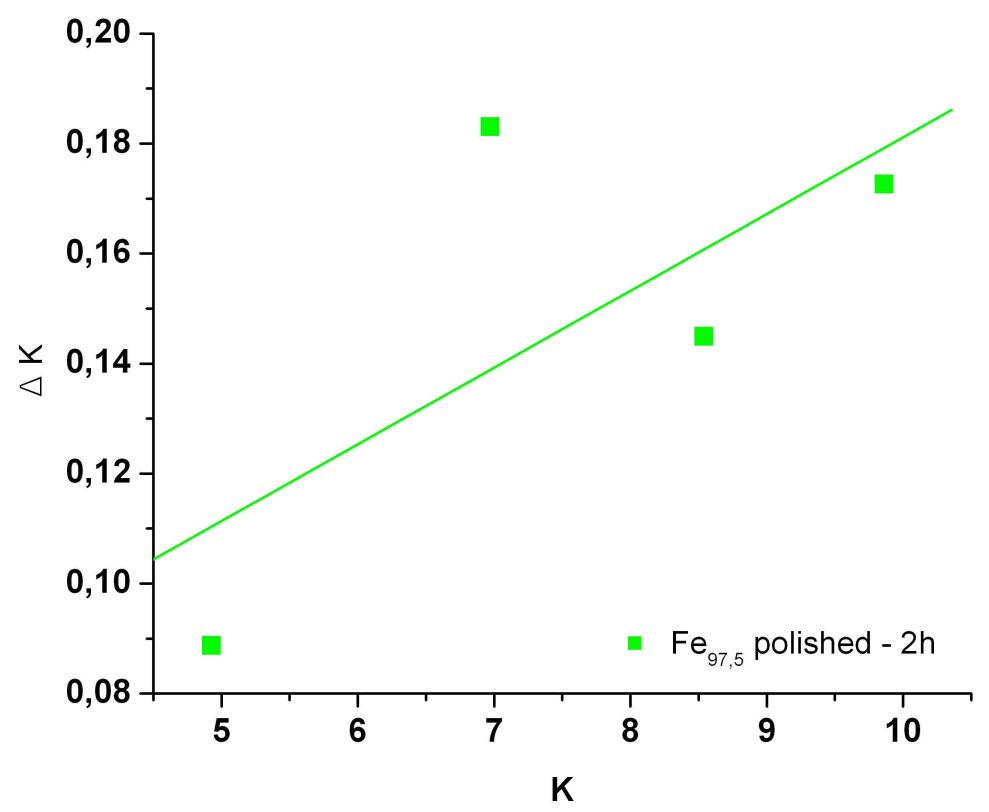

Figure 4.14: Williamson-Hall-plot for Fe-2.5 at.\% Cu after $2 \mathrm{~h}$ milling time, clear deviation of the $\mathrm{K}$ value of the second diffraction reflex (200) from the expected linear relationship

inconclusive [Williamson53]. Figure 4.14 exemplarily shows the Williamson-Hall-plot for Fe2.5 at.\% $\mathrm{Cu}$ after $2 \mathrm{~h}$ milling time. The (200) peak clearly deviates most from the assumed linear relationship between $\Delta \mathrm{K}$ and $\mathrm{K}$. This deviation does not only occur for this sample but for all $\mathrm{Cu}$-concentrations and milling times systematically and in the same manner. The linear regression always results in a negative ordinate intercept. But, as the ordinate intercept corresponds to the mean grain size, it does not seem reasonable to apply this analysis to these samples.

Instead, the modified Williamson-Hall-analysis as proposed by Ungár was applied. As described in the theory, the absorption ability of dislocation is accounted for by means of a contrast factor. Following this modified Williamson-Hall-analysis, the contrast factor can be determined for $\mathrm{Fe}-2.5$ at.\% $\mathrm{Cu}$ after $2 \mathrm{~h}$ milling time.

The elastic constants are approximated by the ones for pure Fe: $c_{11}=234 \mathrm{GPa}, c_{12}=136 \mathrm{GPa}$ and $c_{44}=118 \mathrm{GPa}$ and combined in the elastic anisotropy $\mathrm{S}=2 \cdot \frac{c_{44}}{c_{11}-c_{12}}=2.41$ and the ratio $\frac{c_{12}}{c_{44}}=1.15$ [Ashcroft02]. According to Eq. 3.12 this leads to $\mathrm{C}_{h 00}=0.256$. The experimentally determined value of $q$ is 2.299 . This leads to the general contrast factor of

$$
\mathrm{C}=\mathrm{C}_{h 00} \cdot\left(1-q \cdot \mathrm{H}^{2}\right)=0.256\left(1-2.299 \cdot \mathrm{H}^{2}\right)
$$




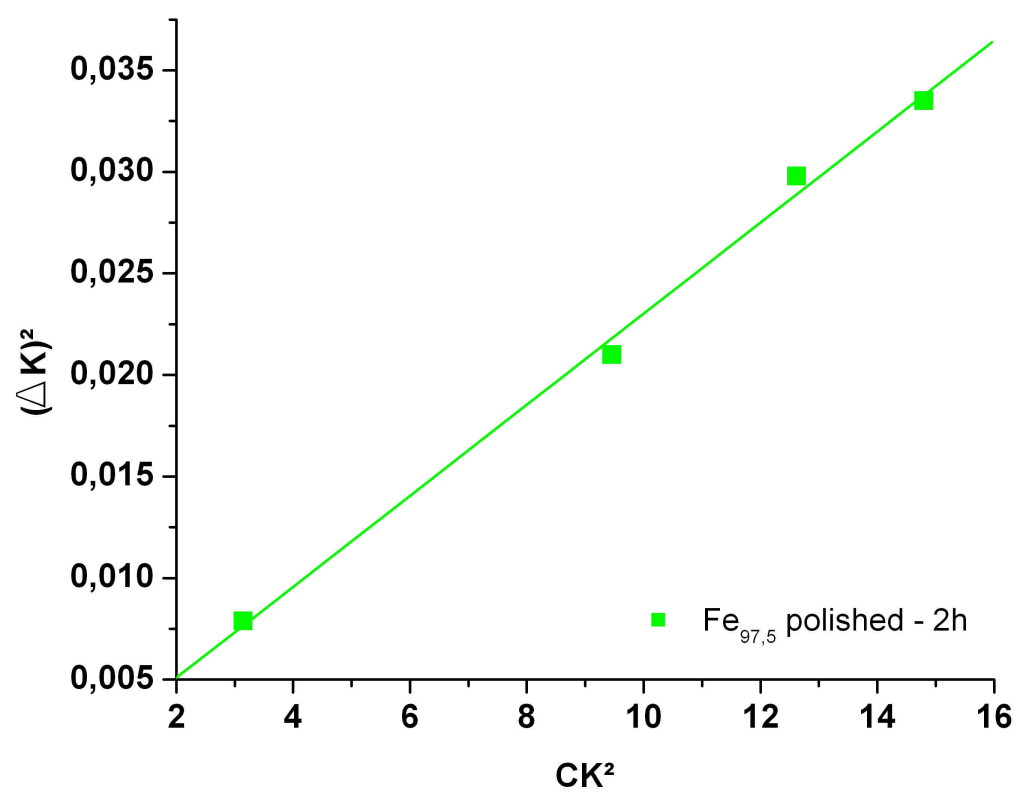

Figure 4.15: Modified Williamson-Hall-plot for Fe-2.5 at.\% Cu after $2 \mathrm{~h}$ milling time, no more deviation of the $\mathrm{K}$ value of any diffraction reflex from the expected linear relationship

with the respective $\mathrm{H}^{2}$ for the different diffraction reflexes. The resulting modified Williamson-Hall-plot in Fig. 4.15 shows a very good linear dependence of $\Delta \mathrm{K}$ on $\mathrm{CK}^{2}$. The ordinate intercept again is a measure of the grain size. For Fe-2.5 at.\% $\mathrm{Cu}$ after $2 \mathrm{~h}$ milling time an average grain size of $36 \mathrm{~nm}$ could be determined. Comparing this result with the one derived by means of Scherrer's formula, the deviation is significant: $D_{\text {Scherrer }}=12 \mathrm{~nm}$ and $\mathrm{D}_{\mathrm{WH}}=36 \mathrm{~nm}$.

It becomes clear that the deconvolution of size and strain effects to peak broadening is essential in the analysis of ball milled material. Utilising only Scherrer's formula, the grain size will be vastly underestimated by a factor of at least $\frac{D_{\text {Scherrer }}}{D_{W H}}=3$.

The modified Williamson-Hall-analysis of the remaining samples was inhibited by the fact, that the standard error of the linear fit could not be minimised by varying the value of alpha. Instead, the standard error of the linear fit decreased with increasing grain size asymptotically approaching the minimum error.

Therefore, it can only be assumed, that a similar relationship between $\mathrm{D}_{\text {Scherrer }}$ and $\mathrm{D}_{\mathrm{WH}}$ applies for the other samples as well. For the samples which were exposed to longer milling, the factor could be even higher, as the strains in these samples are at least as high as in the one milled for only $2 \mathrm{~h}$. 


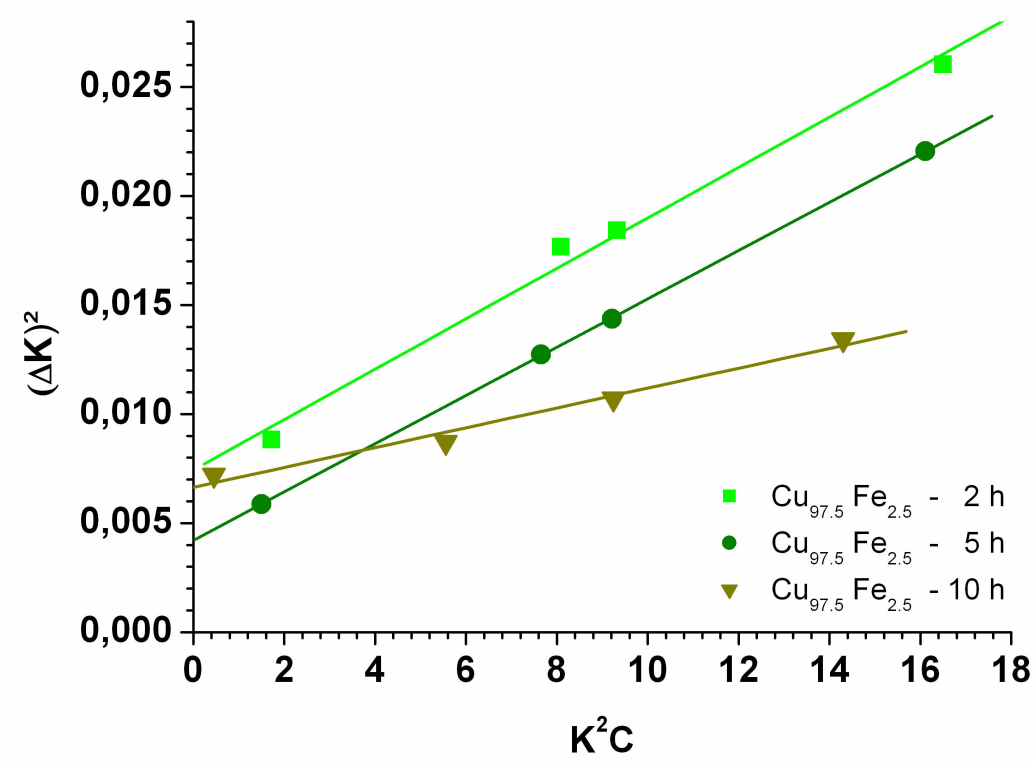

Figure 4.16: Modified Williamson-Hall-plot for Cu-2.5 at.\% Fe, very good linear relationship for all milling times [Schmidt07]

The strain can only be evaluated by the method of Fourier analysis. This analysis was not performed within this work. But, for $\mathrm{Fe}-20$ at.\% Cu Shen et. al. performed the modified Williamson-Hall analysis after Ungár [Shen05]. As they obtained similar values for the dislocation contrast factor and the parameter q, their values for the dislocation density will be comparatively referred to. After $30 \mathrm{~h}$ milling in a SPEX 8000 mill they obtained a gran size of $\approx$ $10 \mathrm{~nm}$ and a corresponding dislocation density of $\rho \approx 6 \cdot 10^{16}$ which is very close to the theoretical limit.

Finally, Fig. 4.16 shows the modified Williamson-Hall-plot for $\mathrm{Cu}-2.5$ at.\% Fe after 2, 5 and $10 \mathrm{~h}$. In the case of the $\mathrm{Cu}$-rich alloys the data were not obtained from embedded samples, but from the pristine particles. The minimisation method could be applied to all $\mathrm{Cu}$-rich alloys investigated within the associated Diploma Thesis [Schmidt07]. After the shortest milling time of $2 \mathrm{~h}$ grain sizes of 10-22 nm were observed for the different Fe- contents. After the longest milling time of $10 \mathrm{~h}$ the sample with 2.5 at.\% Fe shows a stagnation of the grain size (10 to 12 $\mathrm{nm}$ ) within the standard deviation, while the $\mathrm{Cu}-10$ at.\%Fe sample shows an increase in grain size ( 22 to $38 \mathrm{~nm}$ ) which is clearly outside the statistical error.

Detailed explanations on the mechanisms leading to stagnation or even an increase of grain size in the Cu-rich alloys (Fig. 4.17) can be found in [Schmidt07]. The results on grain sizes as shown in Fig. 4.17) were compared with results obtained by the evualuation method of Scher- 


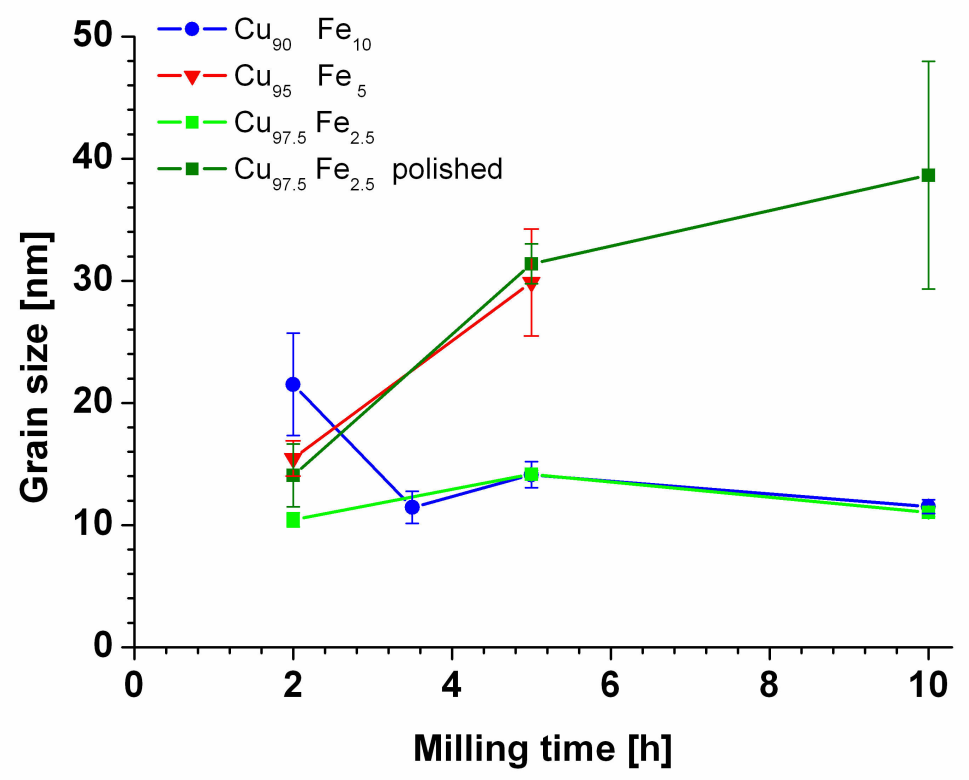

Figure 4.17: Grain size as a function of milling time employing Ungár's method [Schmidt07]

rer. Since strains are not accounted for in this method, the determined grain size strongly depends on the peak choosen (up to $70 \%$ difference between the peaks of one specimen). Ranging from $12-15 \mathrm{~nm}$ after $2 \mathrm{~h}$ milling time to $13-23 \mathrm{mn}$ after $10 \mathrm{~h}$ milling time, a slight tendency towards increasing grain size was found.

Generally, the mechanisms involved in ball milling of the $\mathrm{Cu}$-rich powders might differ from the ones in the Fe-rich powders. As the melting point of $\mathrm{Cu}$ is about $400 \mathrm{~K}$ below the one of Fe, recrystallistaion may play an important role in ball milling of $\mathrm{Cu}$. Especially as the recrystallisation temperature strongly depends on the degree of deformation, the reduction in recrystallisation temperature may be significant in ball milled powders. Although the whole ball mill was cooled by means of water during the milling process, locally anhanced temperatures due to the impacts of the balls cannot be excluded. This would explain the rather large and constant grain sizes obtained for the $\mathrm{Cu}$-rich samples.

Additionally, a slow recrystallisation after the milling might contribute to the large grain sizes observed.

Having obtained an overview of the grain size evolution during ball milling by menas of $\mathrm{XRD}$, the aspects of morphology, texture and dislocation density will be traced in the next section. 
a)

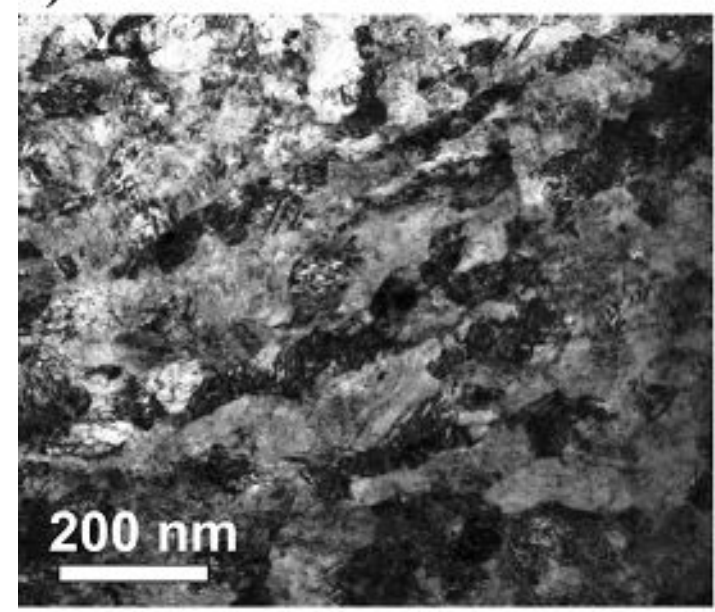

c)

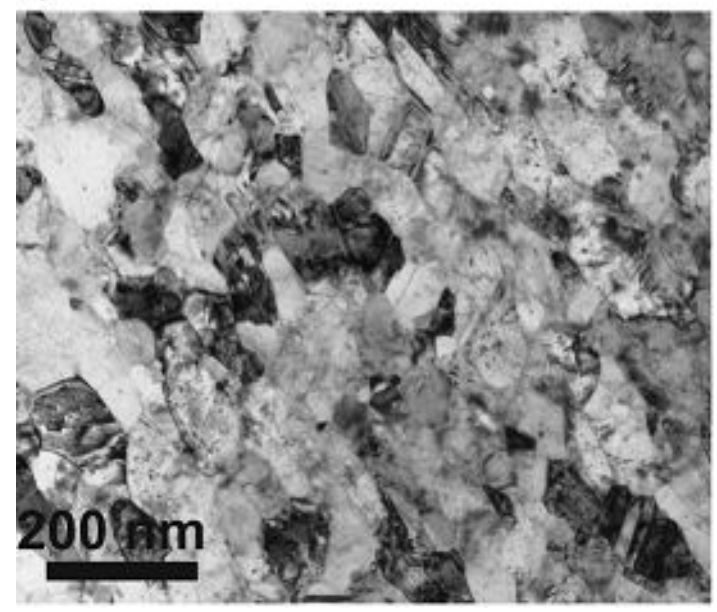

b)

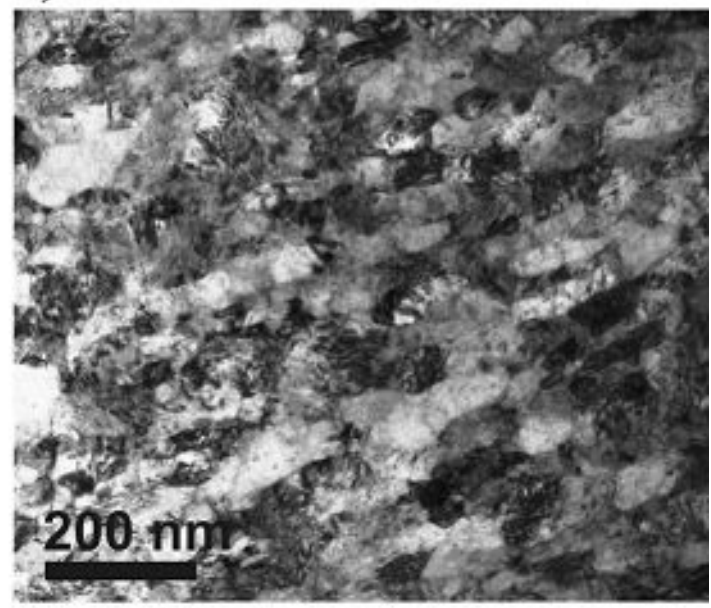

d)

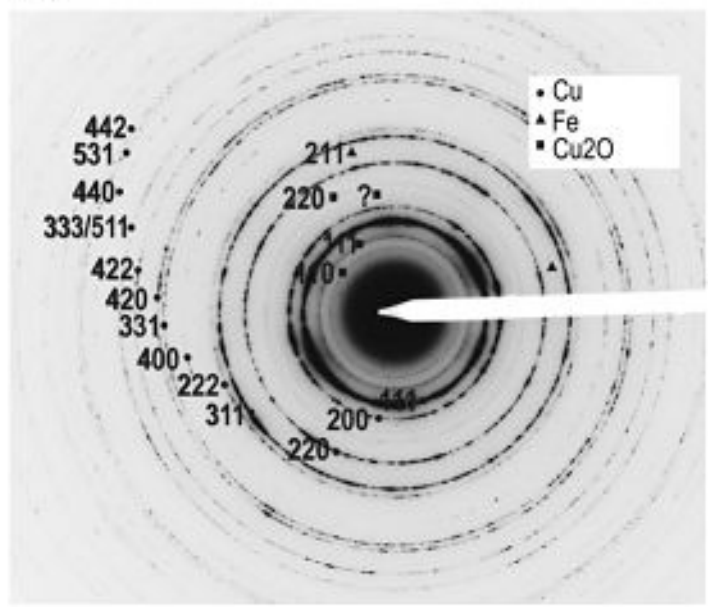

Figure 4.18: BF images of Cu-2.5at.\%Fe: a) after $2 \mathrm{~h}, \mathrm{~b}$ ) after $5 \mathrm{~h}, \mathrm{c}$ ) after $10 \mathrm{~h}$ and d) SAD pattern after $10 \mathrm{~h}$ with single diffraction spots of bcc Fe [Schmidt07]

\subsection{Transmission Electron Microcroscopy}

The structural characterisation of the powders was continued by means of transmission electron microscopy (TEM). The structural information gained by LOM and SEM was complemented by TEM investigations increasing the magnification from 200 up to 340,000 times.

As site specific preparation of TEM lamellae was enabled by the use of FIB, the microstructure could be investigated for the original powder surface as well as for the interior of the particle.

A short overview of the general trends in microstructure in the $\mathrm{Cu}$-rich alloys is given in Figure 4.18. Exemplarily, bright field (BF) images of Cu-2.5at.\%Fe after 2, 5 and $10 \mathrm{~h}$ milling 
time are given in parts a) - d). A slight elongation of the grains parallel to the original particle surface can be found. With increasing milling time the grains become less elongated and after $10 \mathrm{~h}$ milling time hardly any elongation can be found. A rough estimate of the grain sizes was performed measuring 3 grains per state in DF images. Ranging from 125 to $230 \mathrm{~nm}$ after $2 \mathrm{~h}$ milling the grain size slightly increases to a range of 250 to $340 \mathrm{~nm}$ after $10 \mathrm{~h}$ milling. Taking into account the limited field of view and the bad statistics this slight increase could as well be stagnation.

Looking at the SAD pattern of $\mathrm{Cu}-2.5 \mathrm{at} . \% \mathrm{Fe}$ after $10 \mathrm{~h}$ milling supports the assumption formulated in the chapter on light optical microscopy. Even for the longest milling time $(10 \mathrm{~h})$ and lowest Fe-contents single diffraction spots of bcc Fe can be found. This means that not even for the optimum conditions the alloying is completed due to the high ductility of $\mathrm{Cu}$ and the associated lack in fracturing during ball milling. More detailed information on the Cu-rich samples can be found in [Schmidt07].

A completely different picture arises again when looking at the Fe-rich alloys. Figure 4.19 shows the microstructure of a) Fe-2.5at.\%Cu after $2 \mathrm{~h}$ and $50 \mathrm{~h}, \mathrm{~b}$ ) Fe-5at.\%Cu after $2 \mathrm{~h}$ and $20 \mathrm{~h}$, and c) Fe-10at.\%Cu after $2 \mathrm{~h}$ and $10 \mathrm{~h}$. The short milling time is displayed on the left, the long one at the right side, respectively. For all compositions the sacrificial Pt layer has been imaged as well to document the location of the image. For the short milling time all compositions exhibit a clearly lamellar structure. This reflects the findings of flaky powder particles in the SEM images. But, the higher the $\mathrm{Cu}$-contents the higher is the frequency of single big grains.

After the respectively longest milling time the microstructure has changed significantly for all samples. The formerly elongated grains developed a nearly equi-axed shape. The most pronounced effect was found for Fe-2.5at.\%Cu. It is assumed that this rounding of the grains is due to repeated fracturing and impacts from different directions. As the material with the lowest amount of the minority component shows the biggest decrease of the aspect ratio, the fracturing seems to be most effective in this material supporting the assumption of increasing ductility with increasing $\mathrm{Cu}$-content.

In the TEM micrographs of the Ag-Cu system, which as well as $\mathrm{Fe}-\mathrm{Cu}$ exhibtis a positive heat of mixing, Uenishi et. al. [Uenishi91] found the same lamellar microstructure after ball milling of $2 \mathrm{~h}$ as shown in this work in Figs. 4.19 and 4.20. As within this work Uenishi et. al. observed the refinement of the lamellar structure. After $400 \mathrm{~h}$ the lamellae disappaered and instead a homogeneous fine structure with a grain size of $10 \mathrm{~nm}$ was detected in TEM. This observation on the Ag-Cu system will be traced for our system in the following. 
a)

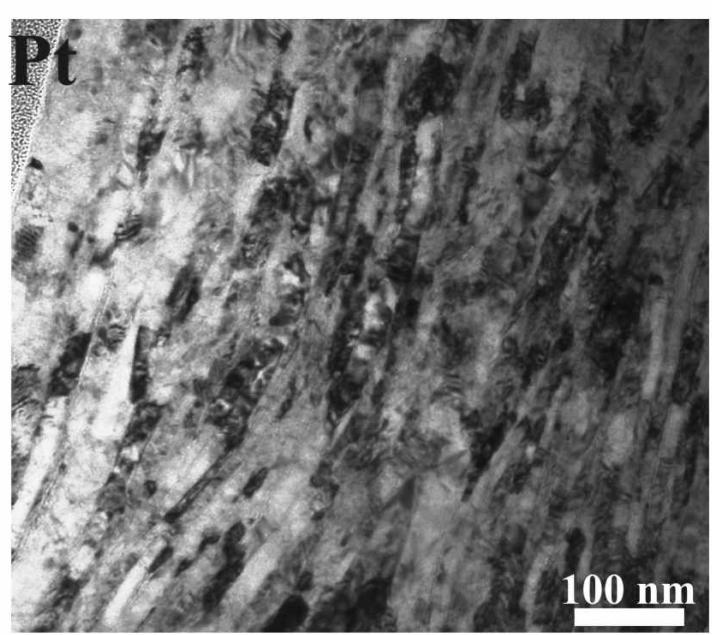

b)

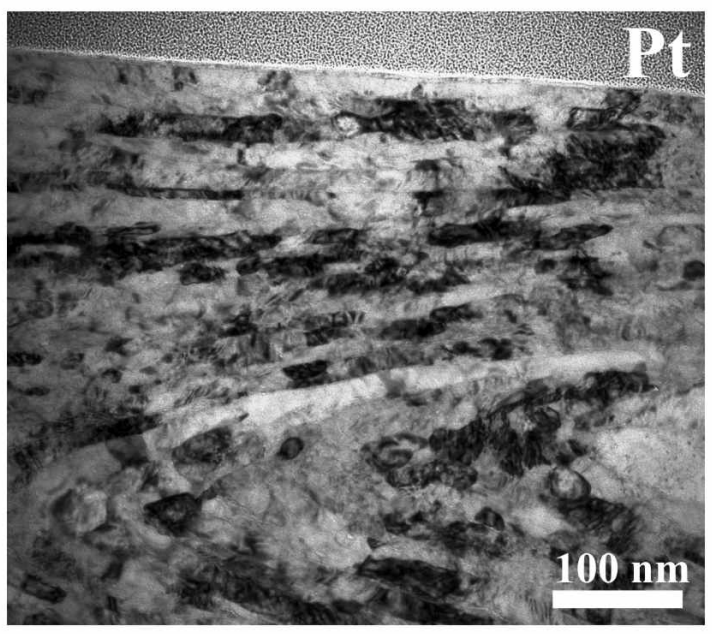

c)

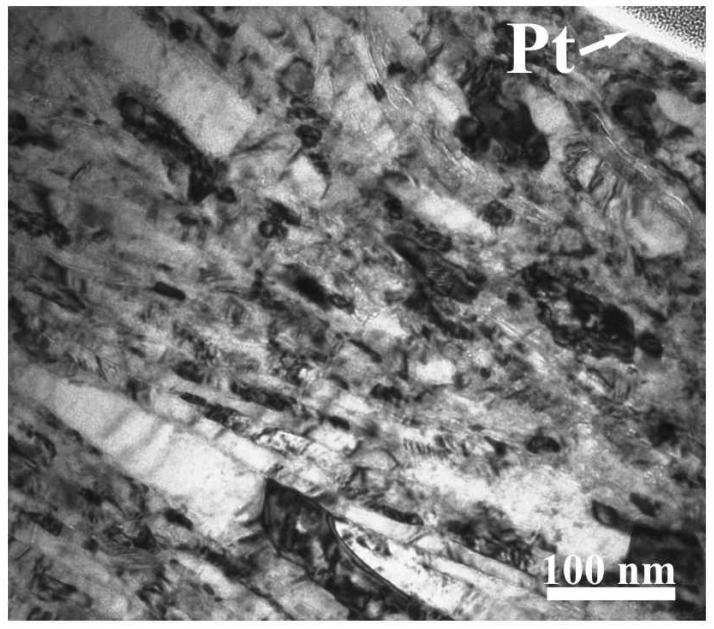

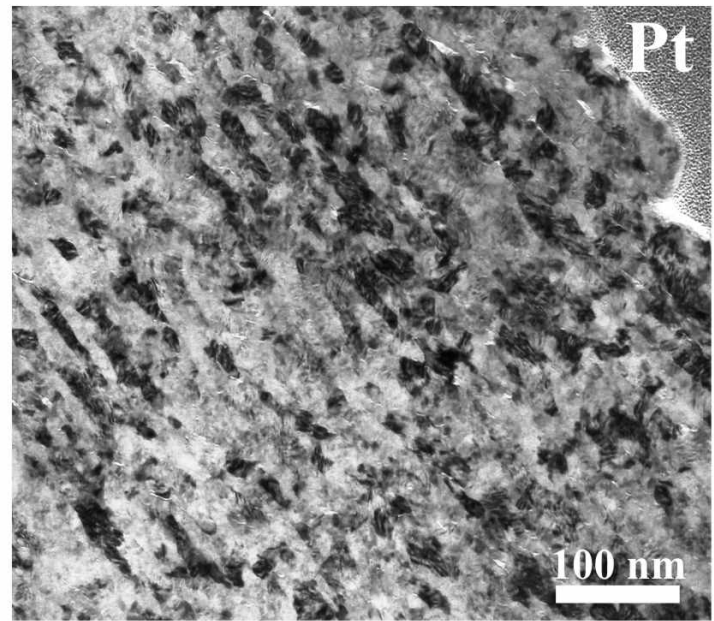
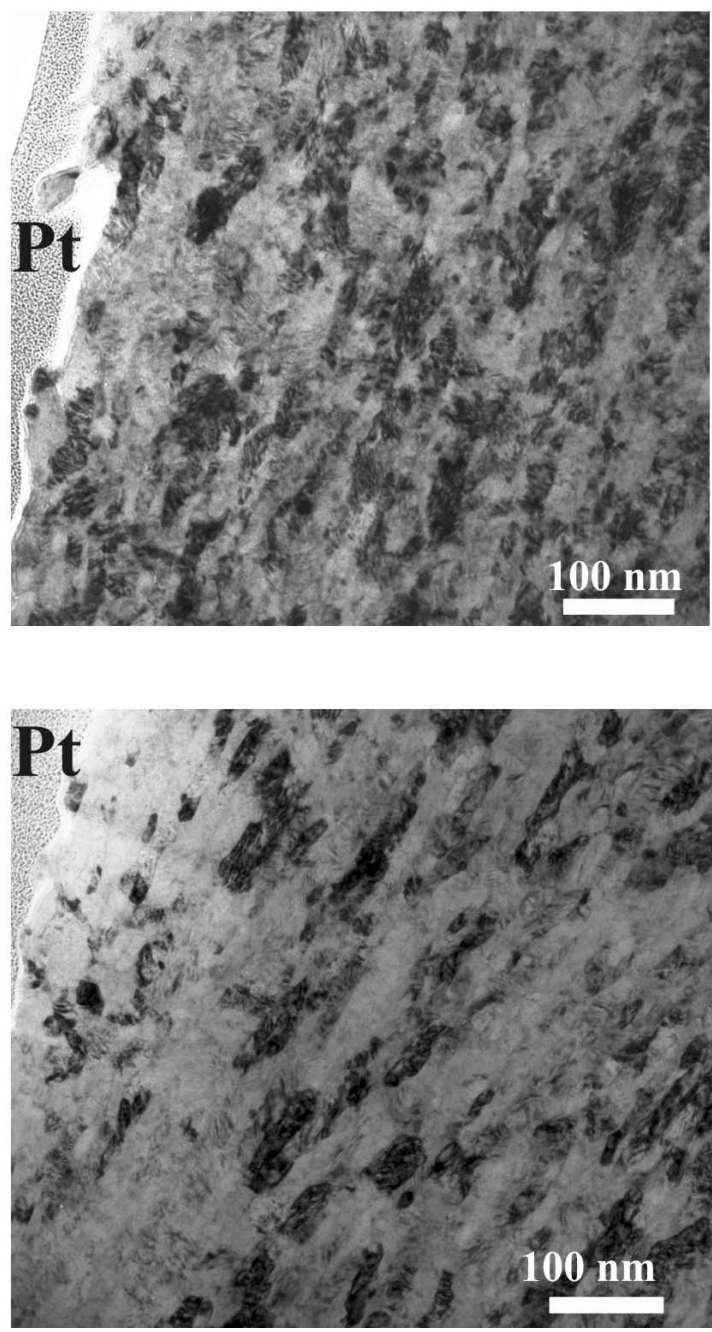

Figure 4.19: TEM BF images of the surface region of the particles of a) Fe-2.5at.\%Cu after $2 \mathrm{~h}$ and $50 \mathrm{~h}$, b) Fe-5at.\%Cu after $2 \mathrm{~h}$ and $20 \mathrm{~h}$, and c) Fe-10at.\%Cu after $2 \mathrm{~h}$ and $10 \mathrm{~h}$ 
a)

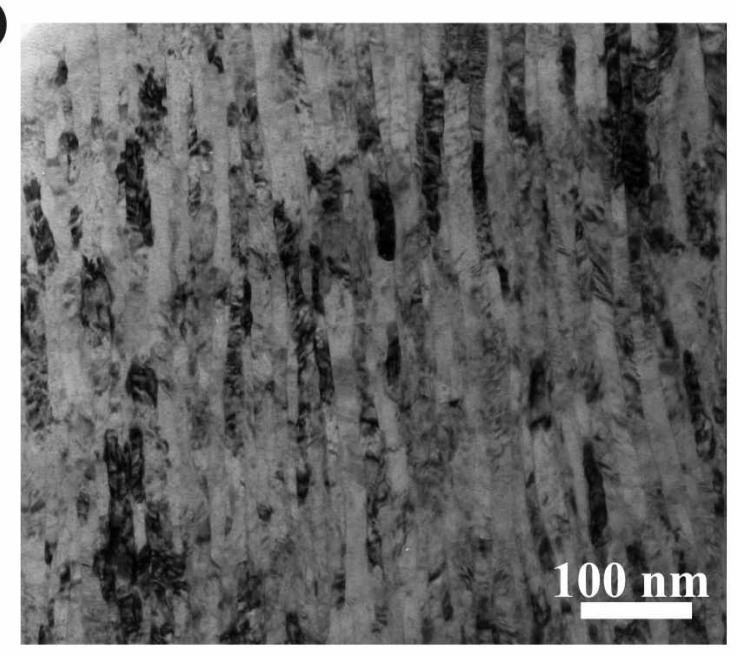

b)

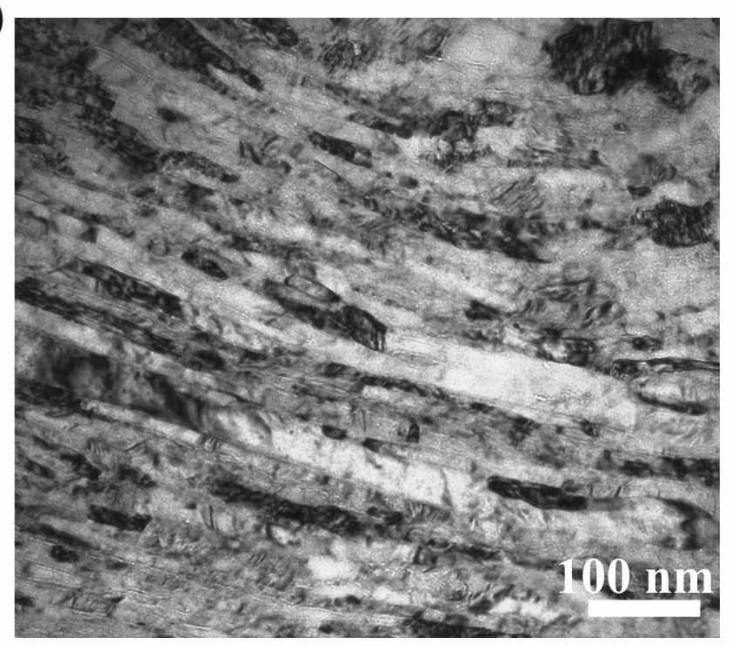

c)

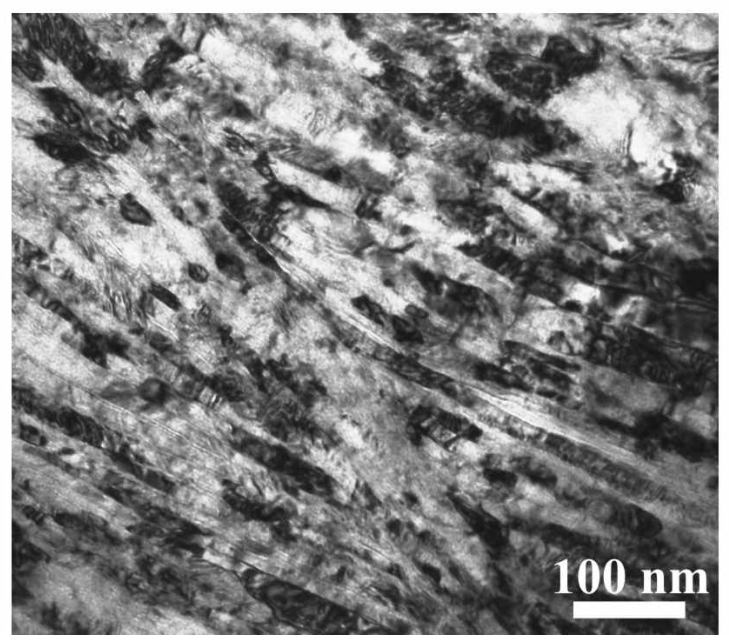

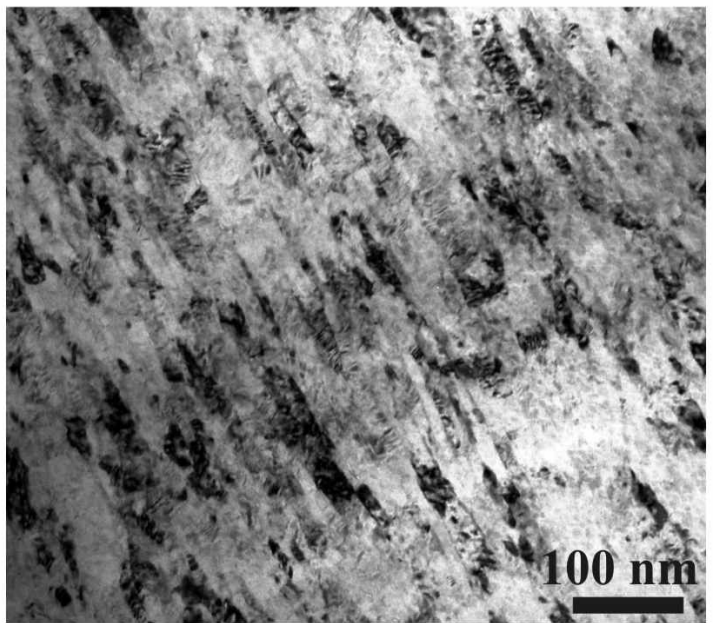
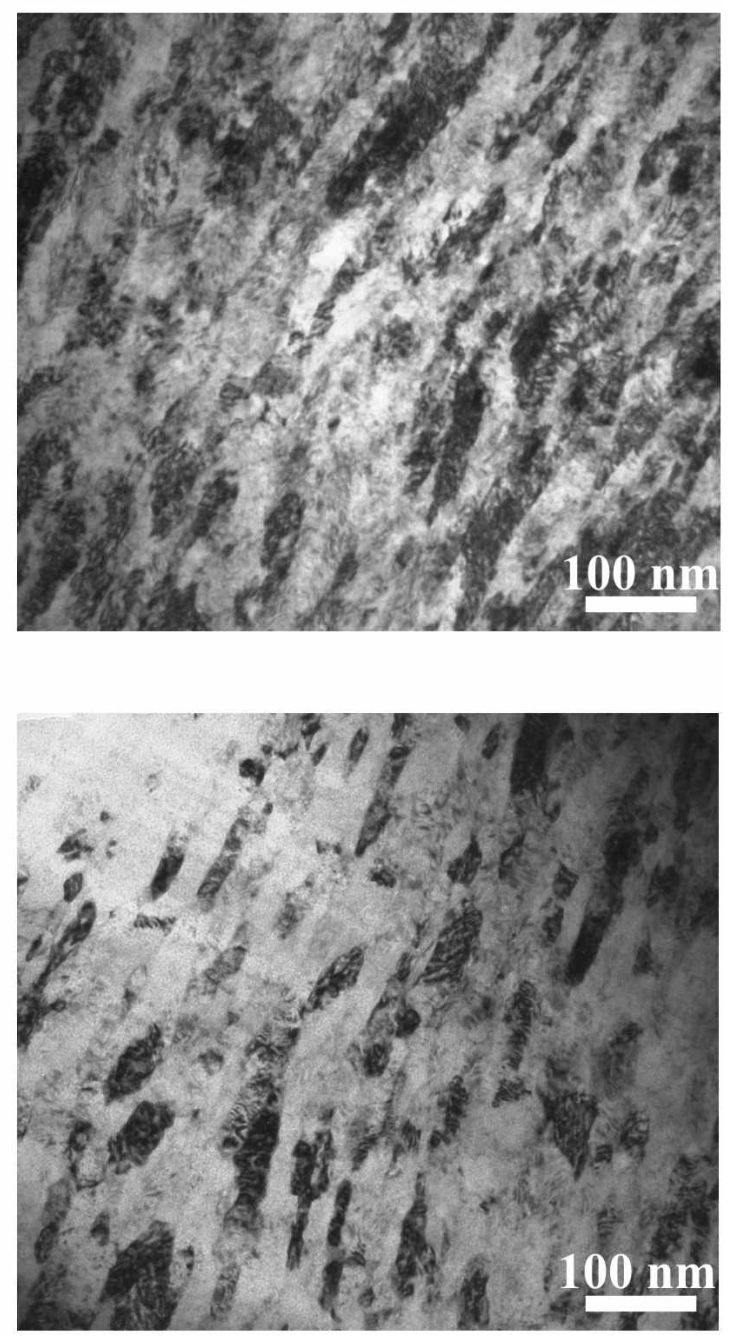

Figure 4.20: TEM BF images of the inner region of the particles (in several $\mu \mathrm{m}$ depth) of a) $\mathrm{Fe}-2.5 \mathrm{at} . \% \mathrm{Cu}$ after $2 \mathrm{~h}$ and $50 \mathrm{~h}, \mathrm{~b}) \mathrm{Fe}-5$ at.\%Cu after $2 \mathrm{~h}$ and $20 \mathrm{~h}$, and c) Fe-10at.\%Cu after $2 \mathrm{~h}$ and $10 \mathrm{~h}$ 


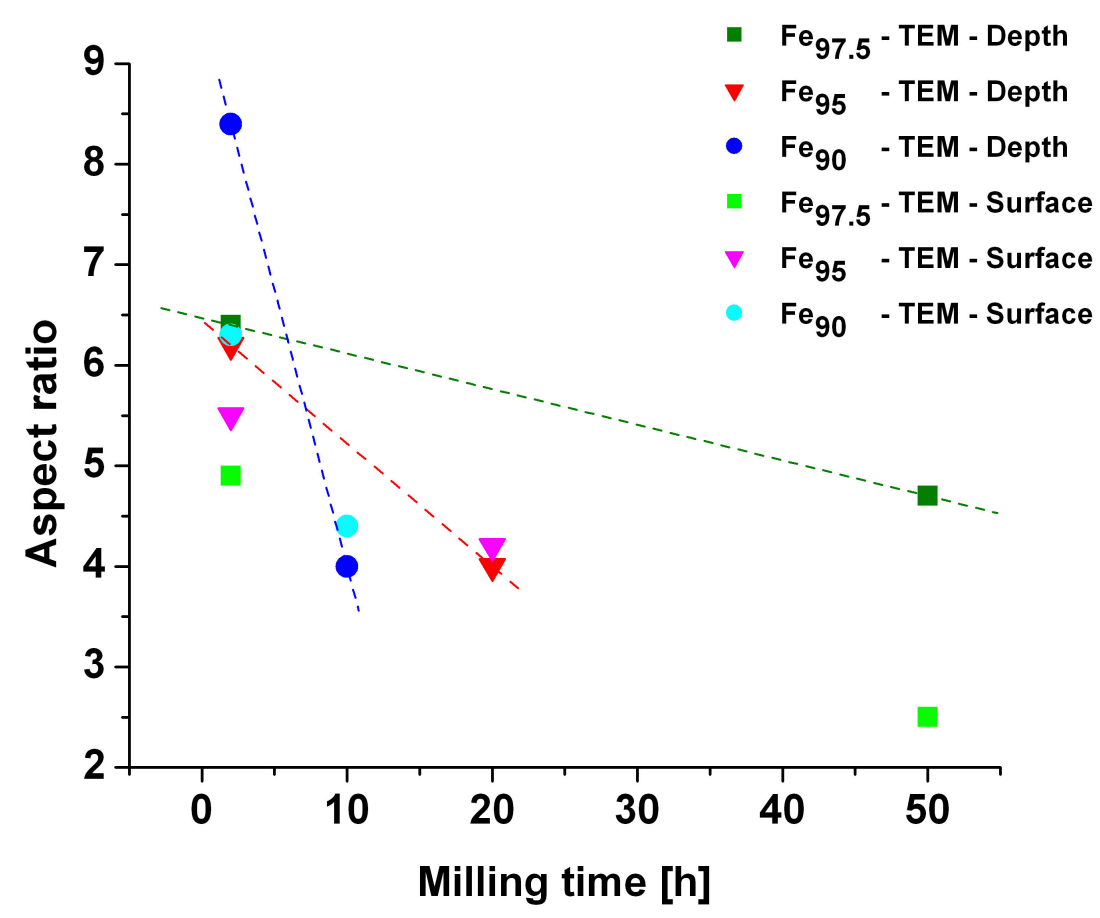

Figure 4.21: Aspect ratio is plotted versus milling time for all Fe-rich samples. Separate evaluation of depth and surface region shows the same qualitative behaviour for all concentration: aspect ratio decreases with milling time

Advancing from the surface to the interior of the particle a different microstructural picture arises. As can be seen in Figure 4.20, several micrometres inside the particle the lamellae are even more pronounced then at the surface after $2 \mathrm{~h}$ milling time. The aspect ratio in the inner region is always higher then the one for the same state at the surface. As the milling proceeds, in the inner region of the particles the same process can be found as at the surface. The aspect ratio of the grains decreases with increasing milling time. But, although the increase is more intensive then at the surface the grains stay elongated and the lamellar microstructure is maintained as the initial aspect ratio was much higher.

These trends were quantified by measuring at least 100 grains per state. The values of the aspect ratios are plotted versus milling time in Fig. 4.21. It can be seen that not only the decrease in aspect ratio is less pronounced but also the initial aspect ratio. For Fe-10at.\%Cu and $\mathrm{Fe}-5 \mathrm{at} . \% \mathrm{Cu}$ this combination leads to a convergence of aspect ratio in the depth and at the surface of the particle for long milling times.

After the respectively longest milling time the aspect ratio at the surface and the one in the depth are the same with the accuracy of measurement (blue circles and red triangles). Thus it 
a)
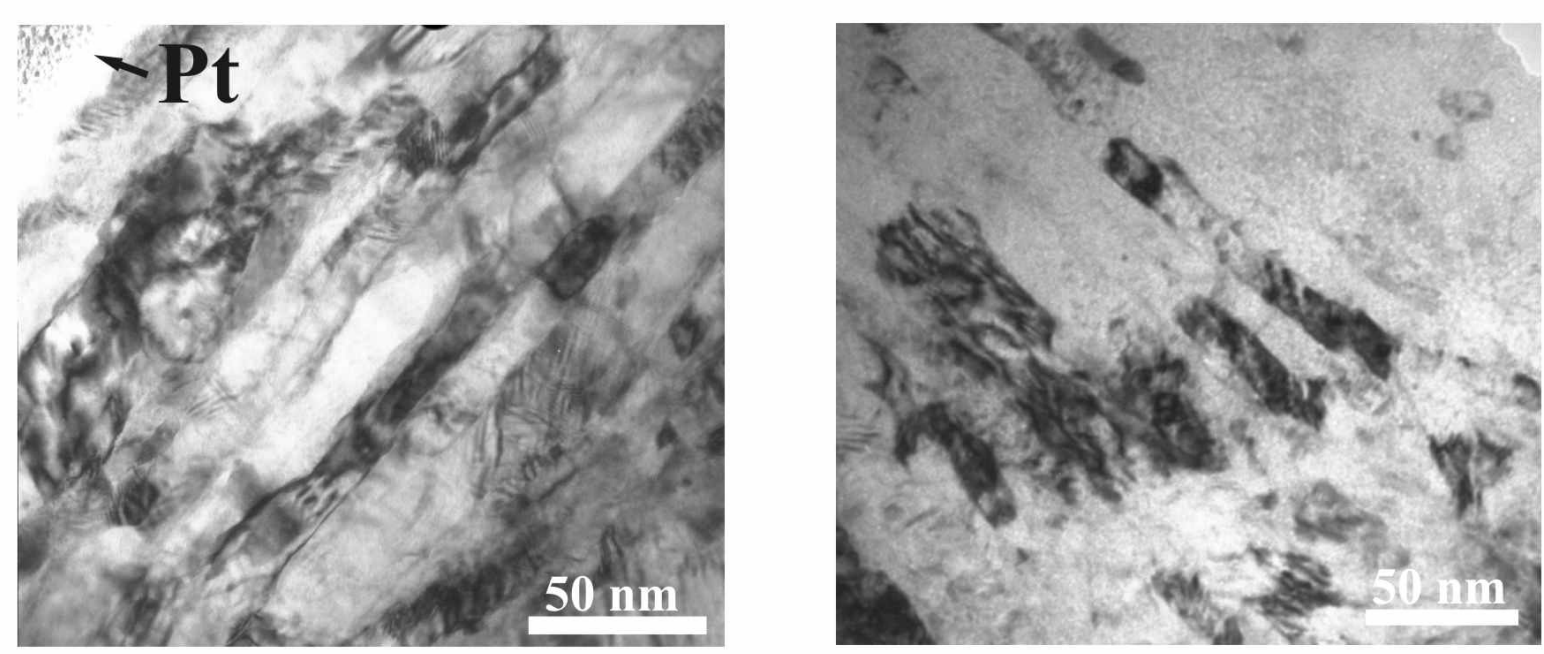

b)
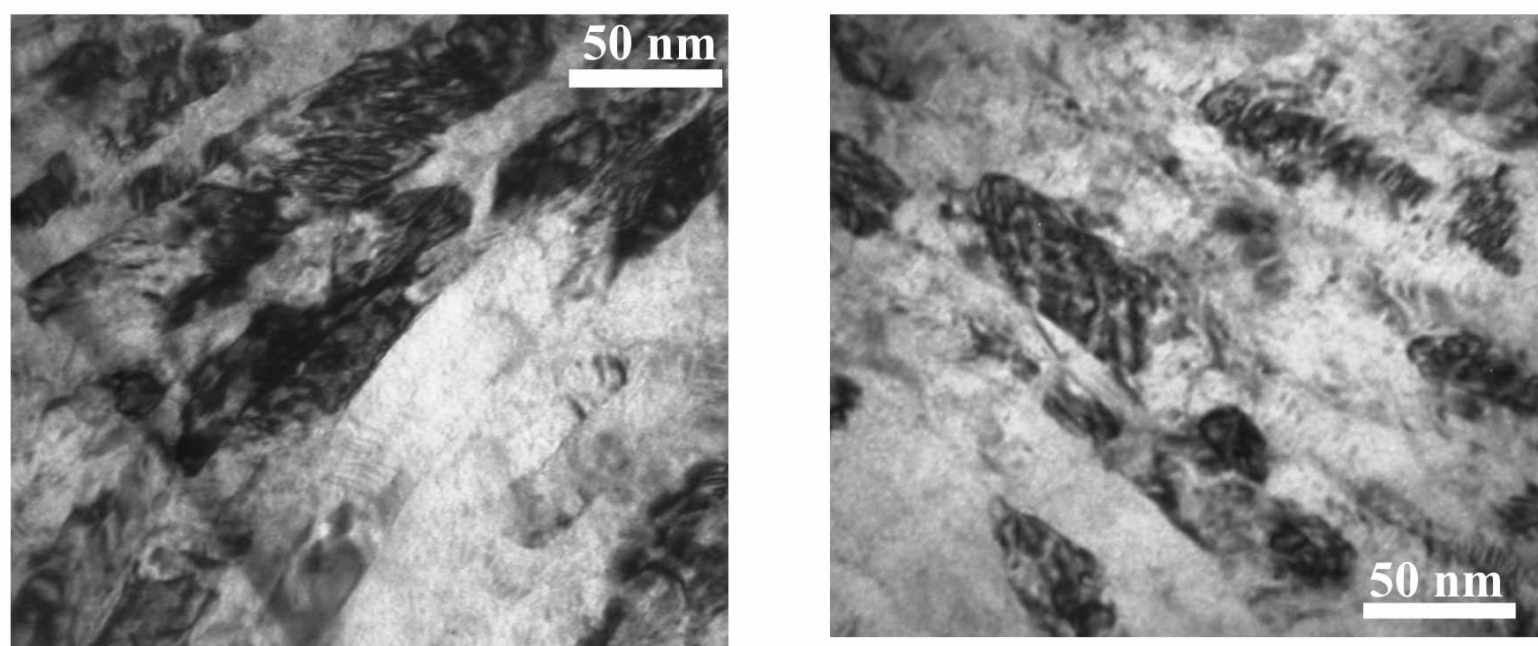

Figure 4.22: TEM BF images at higher magnification (340kx) of Fe-10at.\%Cu a) surface region and b) inner region of the particles (in several $\mu \mathrm{m}$ depth) after $2 \mathrm{~h}$, respectively

can be assumed that after long enough milling times the particles of the Fe- rich powders are microstructurally homogeneous on the length scale of some 10 nanometres. The chemical homogeneity will be evaluated and discussed as well beneath in this chapter as in the chapter on atom probe tomography.

Increasing magnification from 120,000 to 340,000 times, Figure 4.22 shows the structure within the single grains. Exemplarily the surface and inner region of $\mathrm{Fe}-10 \mathrm{at} . \% \mathrm{Cu}$ is shown after $2 \mathrm{~h}$ milling, respectively. Even after the shortest milling time of $2 \mathrm{~h}$ the grains in all parts of the lamella show heavy strain contrast and a dislocation density near the theoretical limit can be assumed. This is in agreement with the dislocation density determined by Shen et al. 
using the modified Williamson-Hall analysis in XRD [Shen05].

As mentioned above, the higher the $\mathrm{Cu}$-content the higher the frequency of single big grains deviating from the overall microstructure. Fig. 4.23 shows a coarser grained region near the surface in Fe-10at.\%Cu after $2 \mathrm{~h}$ milling embedded in the otherwise nanocrystalline lamellar matrix. The lamellae in the overview image in a) do not exhibit one direction of elongation for all grain but two different directions of elongation. The lamellae in the lower left part (I) of the image run from the left downwards to the right. The lamellae in the upper right part (II) span rather diectly downwards with only a slight curvature. The coarser grained region (III) might stem from a particle impinging on the already lamellar microstructure of the big particle from the left side. All three regions are devided by red dotted lines as a guide to the eye.

Zooming into the coarser grained region (III) a single grain of about $200 \mathrm{~nm}$ length (marked by red circle) can further be analysed. This darkly imaging grain exhibits heavy strain contrast. Therefore, it has been subjected to several impacts and fracture events most probably both before and after impinging on the big particle. The dark field (DF) image in c) confirms the darkly imaging region in $\mathrm{b}$ ) to be a single grain.

Dark field images are essential when evaluating grain size in these Fe-rich powders. Most of the grain boundaries are small angle or tilt boundaries. Large angle boundaries are hardly found. Additionally, the heavy strain contrast considerably impedes the identification of grain boundaries in bright field (BF) images. Exemplarily a typical region in Fe-2.5at.\%Cu after $2 \mathrm{~h}$ milling is shown in Fig. 4.24. In the BF image in part a) the darkly imaging region (marked by white oval) might be perceived as a single grain. When looking at DF images in the annular mode, this region can readily be identified to consist of two grains which are tilted by $\approx 10^{\circ}$. Therefore, the determination of grain size from TEM images was conducted by evaluating DF images. But, the limited number of grains available must be named as the main draw back of evaluating DF images. Additionally, the determination of grain size along the short axis was much easier then along the direction of elongation due to the different nature of grain boundaries and the resulting difference in contrast.

For each sample the short axis and long axis were averaged not separating surface and depth. The so derived values for the grain size of all Fe-rich samples are plotted versus milling time in Figure 4.25 b).

Supplementary, the values obtained by the different evaluation methods for XRD are incorporated into the plot in Figure 4.25 a). For Fe- 2.5at.\%Cu dashed lines were included as a guide for the eye. The same trend of a slightly decreasing grain size with milling is found both in XRD and TEM. 
a)

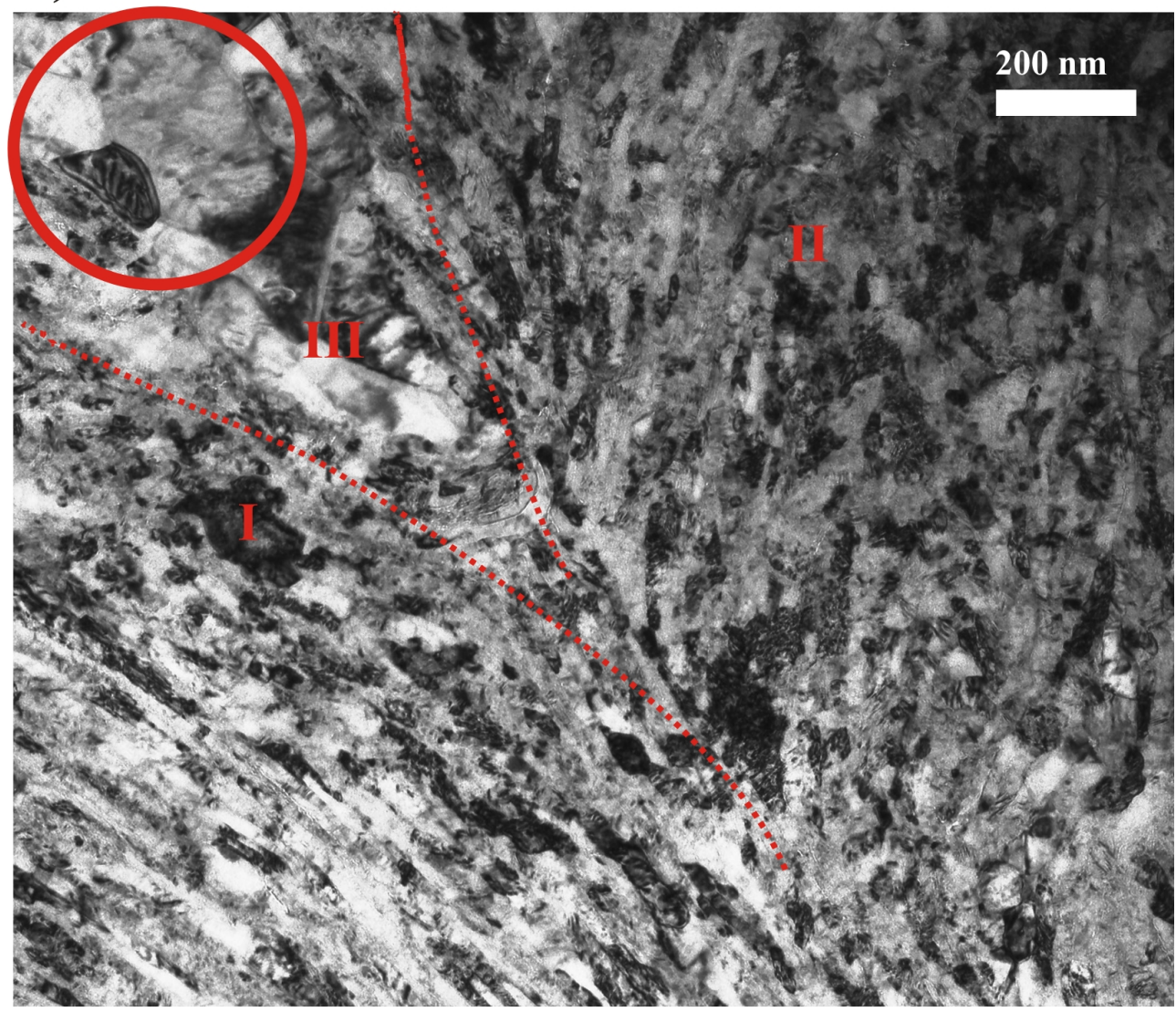

b)

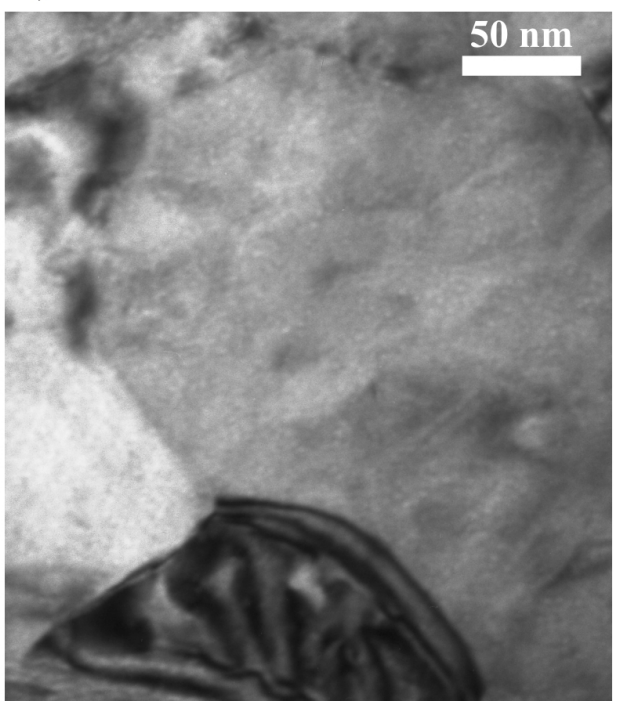

c)

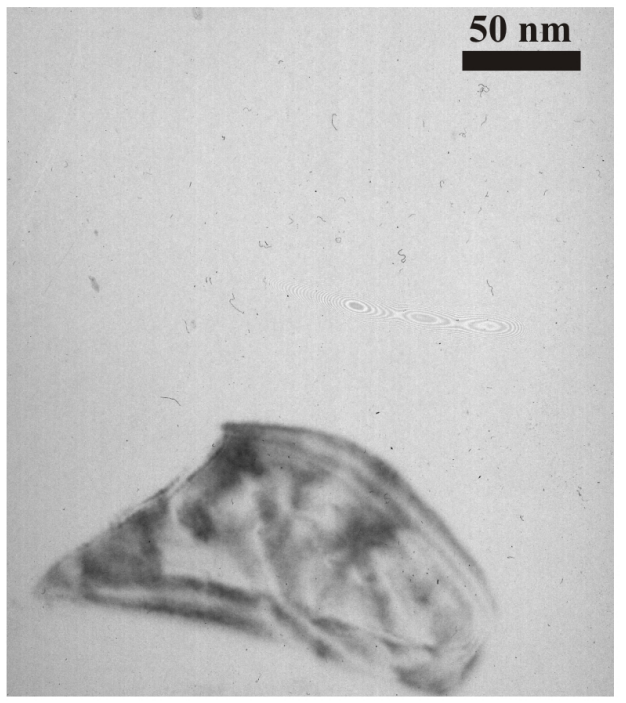

Figure 4.23: TEM of a coarser grained surface region in Fe-10at.\%Cu after $2 \mathrm{~h}$ milling; a) overview, b) BF image and c) DF image showing heavy strain contrast within the single-crystalline region of about $200 \mathrm{~nm}$ length 
a)

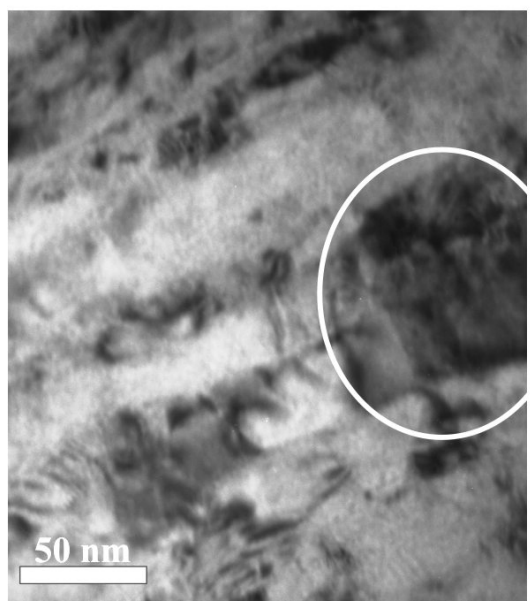

b)

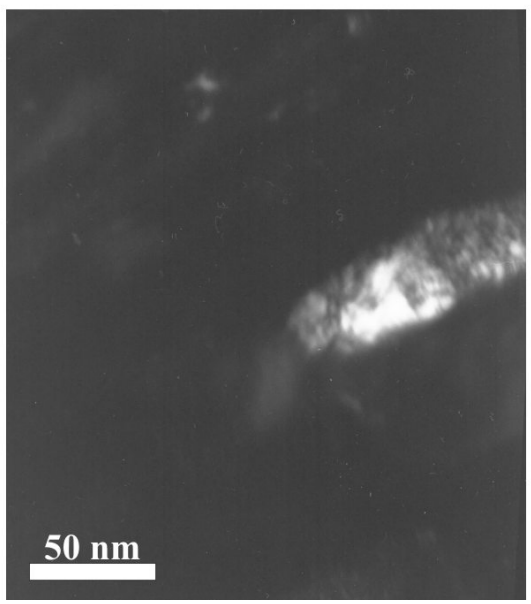

c)

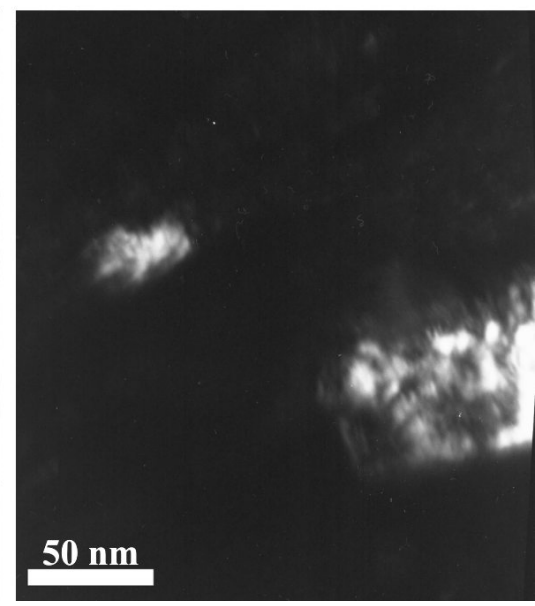

Figure 4.24: TEM of a typical region in Fe-2.5at.\%Cu after $2 \mathrm{~h}$ a) BF image, b) DF image and c) DF image rotated by $\approx 10^{\circ}$, showing the difficulty in the determination of grain sizes from $B F$ images, what seems to a single grain in the $\mathrm{BF}$ image (indicated by white ellipse) turn out to be two tilted grains when consulting the DF images

Even though the TEM data is qualitatively in very good accordance with the XRD data, they differ quantitatively. After only $2 \mathrm{~h}$ milling time the grain size determined by TEM drops to a value below $100 \mathrm{~nm}$ for all samples and the material can be called nanocrystalline by this method as well. When the same state is evaluated by XRD using Scherrer's formula, it yields grain sizes in the range of $10-15 \mathrm{~nm}$. The TEM results are a factor of 4 higher. However, consulting Figure $4.25 \mathrm{~b}$ ) it is striking that the error bars of the values derived by TEM are immense. As the number of grains considered in TEM DF images is much smaller then in the one considered in XRD evaluation, the error bars differ strongly. As can be seen in Fig. 4.10 in the chapter on XRD results, the standard deviation of the grain size is about $4 \mathrm{~nm}$ while ranges from 30 - $60 \mathrm{~nm}$ for the TEM results.

Furthermore, the strain corrected grain size by the modified Williamson-Hall method gives a grain size of $36 \mathrm{~nm}$. This value lies between the value from TEM (48 nm) and the one from XRD without strain correction $(12 \mathrm{~nm})$. Therefore, these values from XRD and TEM are in very good agreement considering the strains.

Consulting the selected area diffraction (SAD) patterns all $\mathrm{Fe}$ - rich alloys are devoid of $\mathrm{Cu}$ rings or even single spots. Exemplarily the development of SAD patterns is shown by means of Fe-2.5at.\%Cu after $2 \mathrm{~h}$ milling time (Fig. 4.26). The SAD patterns were taken at a magnification of 125,000 times. The selected area comprised a circular area with $300 \mathrm{~nm}$ radius.

At the surface, all reflexes are represented by continuous rings. All rings could be indexed to 

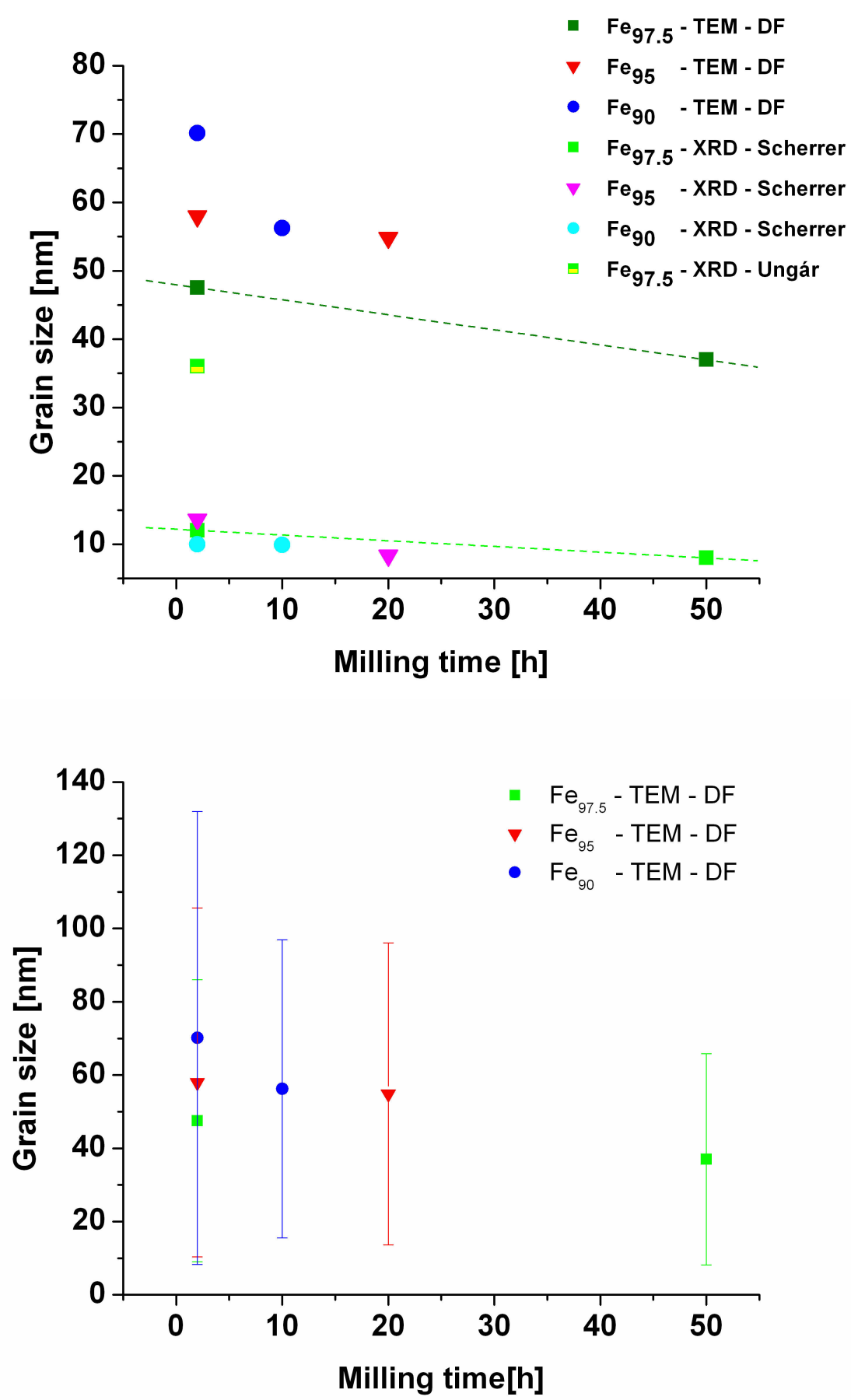

Figure 4.25: a) Grain size is plotted versus milling time for all Fe-rich samples. Grain size decreases with milling time for all samples and methods. b) Only TEM results - including error bars 
a)

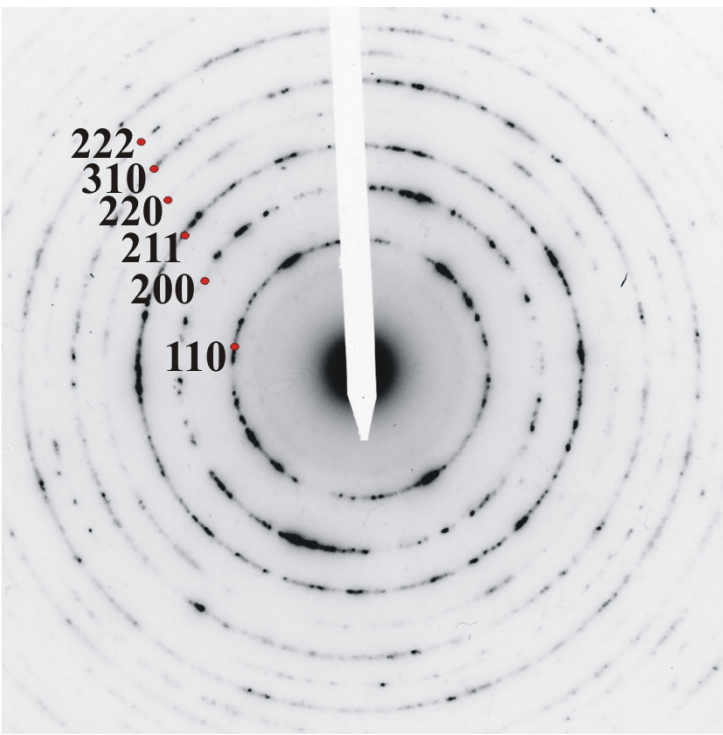

b)

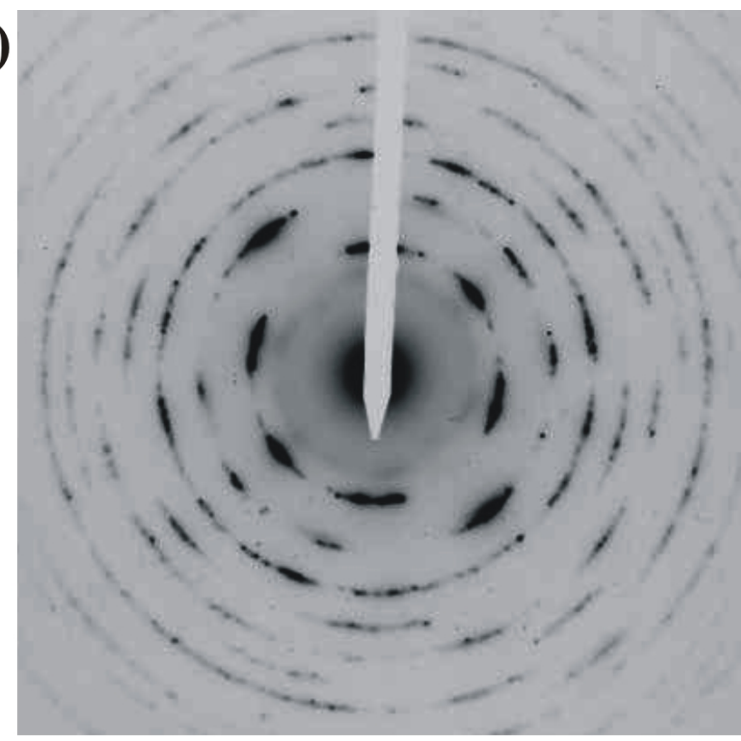

Figure 4.26: SAD pattern of Fe-2.5at.\%Cu after $2 \mathrm{~h}$ milling time a) surface without distinct texture and b) depth with strong rolling texture, indexing the rings yielded only Fe bcc reflexes with perfect relative diameters of the rings

belong to bcc Fe with perfect relative diameters of the rings. But, due to the lamellar structure the preferred orientations manifest themselves in thicker parts of the diffraction rings.

Deeper in the interior of the particle these preferred orientations are even more pronounced in the SAD patterns. The diffraction pattern does not any more consist of continuous rings but of elongated diffraction spot. This is not astonishing as the aspect ratios are 4.9 for the surface and 6.4 for the depth.

The same trend from continuous rings to a spot pattern can be observed for all Fe- rich alloys and all milling times when comparing the surface region with the interior of the particle. The decrease in aspect ratio follows the same trend as well for all states.

Comparing the spot pattern of these ball milled $\mathrm{Fe}-\mathrm{Cu}$ - powders with cold rolled Fe sheets a striking similarity becomes evident. After cross rolling - after each rolling pass the sheet is turned by $90^{\circ}$ - an XRD pattern very similar to Fig. 4.26 evolves. Figure 4.27 shows a DebyeScherrer photograph of a cold rolled Fe sheet exhibiting strong rolling texture at both rolling directions [Wassermann39].

According to Kudjumov and Sachs [Kurdjumov30] the texture of rolled Fe is comprised of three crystall orientations: (100) [011], (112) [110], and (111) [112]. The first two ideal orientations were found to be much more pronounced then the second one. As a direct comparison of the X-ray diffractogramms with the ones obtained by SAD within this work is not possible, the SAD pattern of Fig. 4.26 has been recontructed as a combination of four ideal crystal ori- 


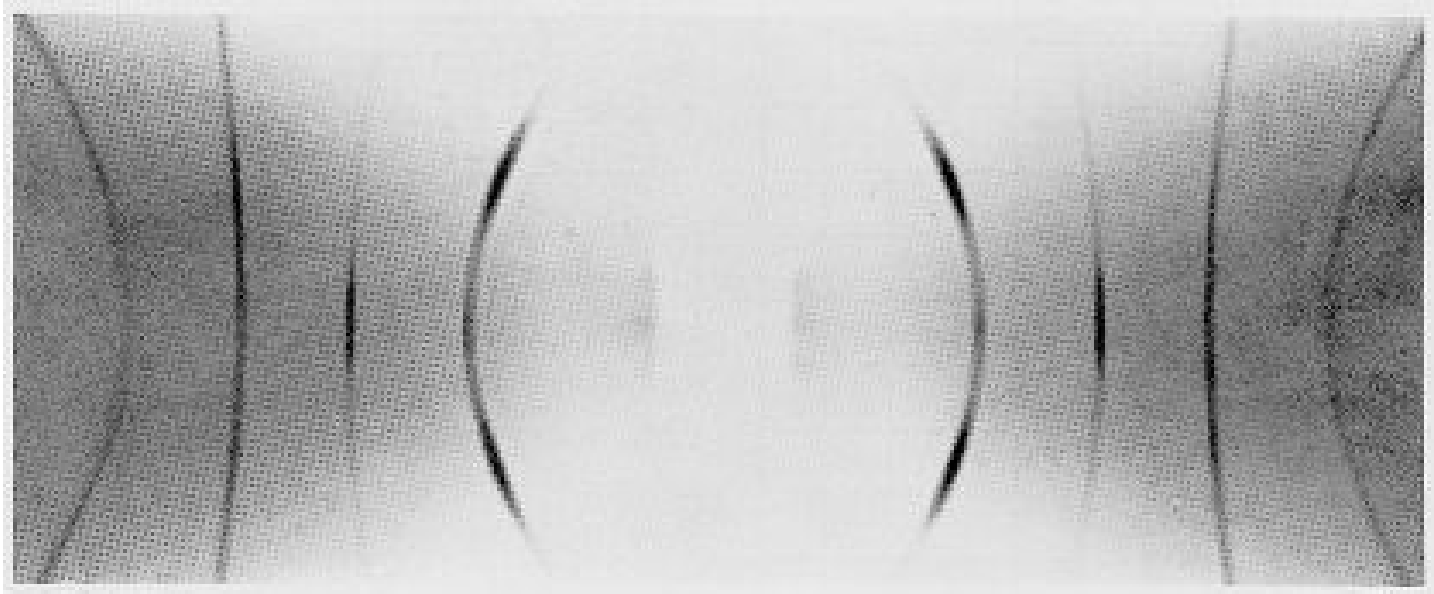

Ablo, 115, Kreuxweis gewalates Blech, 1. WR.

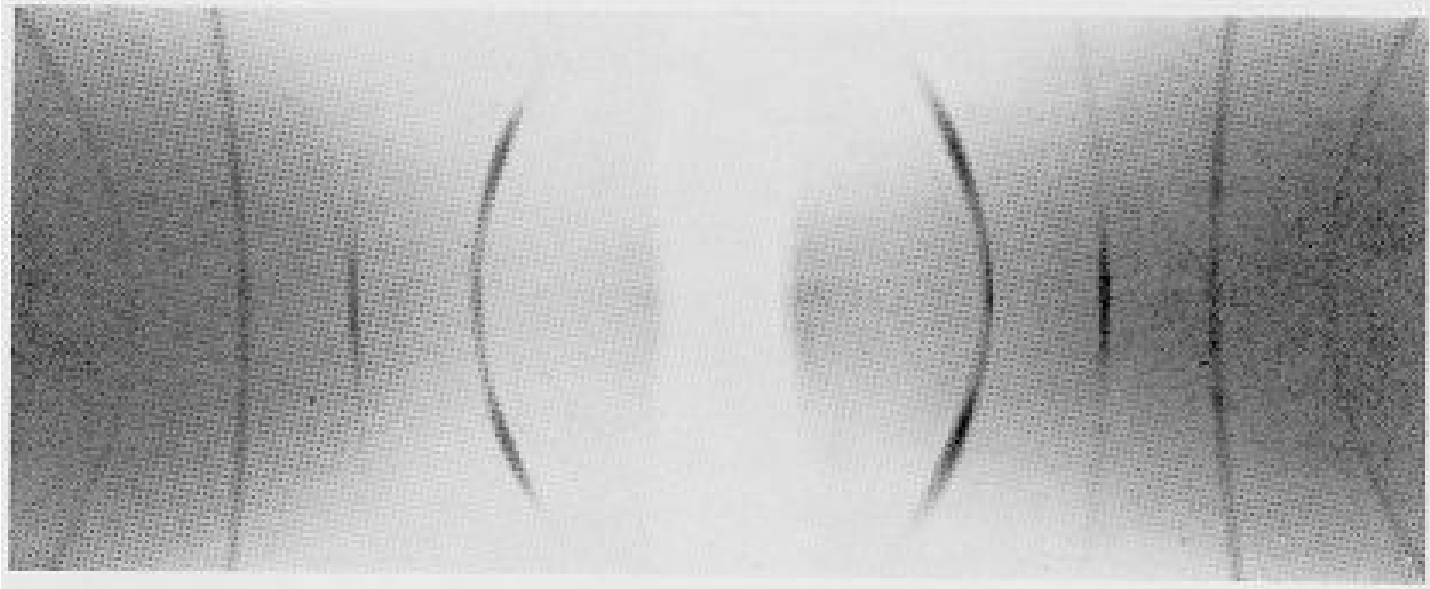

Abh, 116. Kreuxwels gewalxtes Blech, 2. Wht.

Figure 4.27: Debye-Scherrer photograph of cross rolled Fe sheet [Wassermann39] 


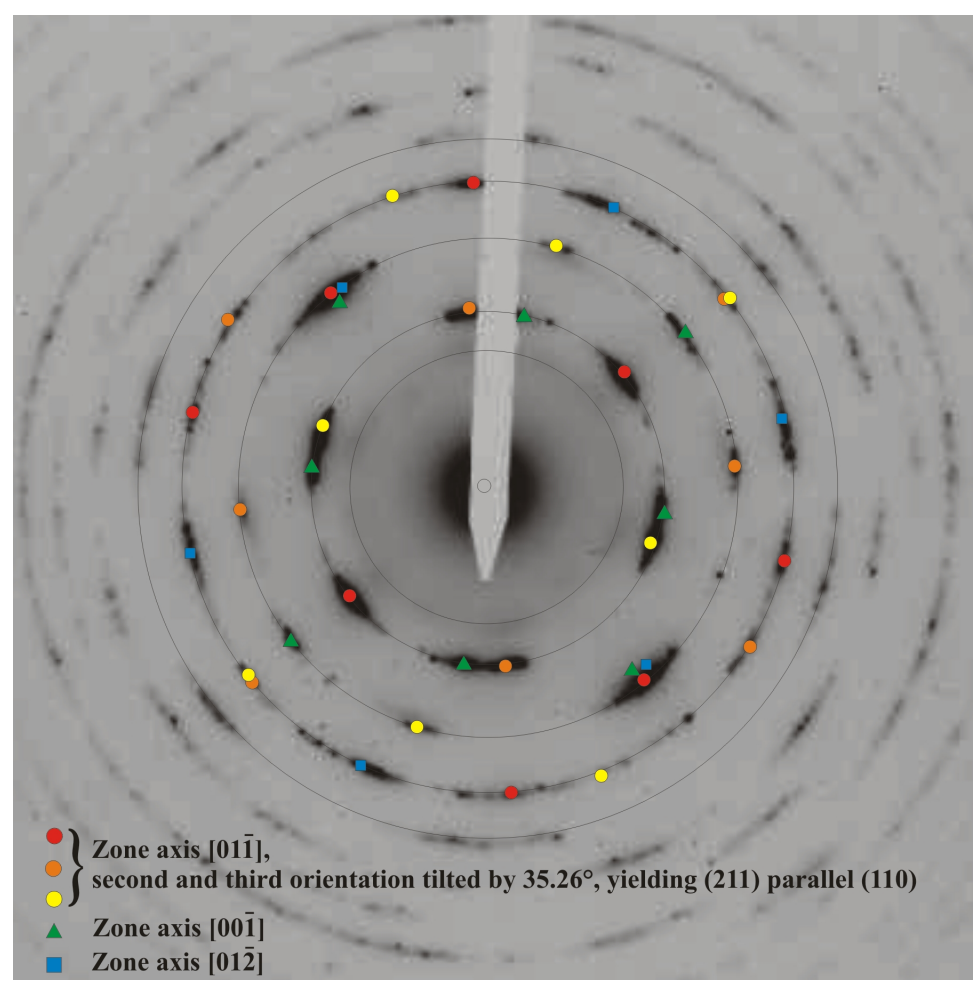

Figure 4.28: $\mathrm{SAD}$ pattern of $\mathrm{Fe}-2.5 \mathrm{at} . \% \mathrm{Cu}$ after $2 \mathrm{~h}$ milling time from the depth of the particle, all major diffraction spots assigned to one of the three ideal orientations as marked by different symbols

entations. The first two components of the texture found by Kurdjumow and Sachs could be confirmed. The (100) [011] orientation leads to three sets of respectively two (110) and (200) diffraction spots around the $[00 \overline{1}]$ zone axis. The second and third set of diffraction spots are rotated by $\pm 35.26^{\circ}$ with respect to the first set, so that the (110) direction of the first set is parallel to the (211) diection of the second and third. The red, orange and yellow circles in Fig. 4.28 each represent one set of diffraction spots.

The second orientation (112) [110] yields four (110) spots mitering in right angles and four (200) spots, which are mitering in right angles as well and include an angle of $45^{\circ}$ with the (110) spots. This ideal orientation is marked by green triangles in Fig. 4.28.

The third component of the texture found by Kurdjumow and Sachs cannot be confirmed here due to the low intensity of the high index diffraction rings. The lower relative intensity of this component was already mentioned by Kurdjumow and Sachs.

In addition to the ideal orietations found by Kurdjumow and Sachs the (100) [121] orientation was found within this work. Labelling this orientation by blue squares all major diffraction spots in Fig. 4.28 could be assigned to one of the three ideal orientations. 
Although the process of ball milling comprises many parameters and it is a stochastic process concerning the deformation of an induvidual particle, the resulting texture can very well be compared to conventionally rolled Fe-sheets. Further investigations on the different regions within a given particle will be needed to clearly associate the deformation processes with the resulting texture.

Closing the section on purely structural aspects of the ball milled Fe-Cu-alloys, in the following the chemical distribution within the grains will be elucidated by means of FIM and APT and compared to the structural information gained by means of XRD and TEM.

\subsection{Field Ion Microscopy and Field Ion Image Tomography}

FIM was chiefly employed to prepare the samples for the subsequent analysis by APT. Surface oxides and damage induced by the Focussed Ion Beam treatment could thus be eliminated.

In this work selected results for the nominal composition of $\mathrm{Cu}-2.5 \mathrm{at} . \% \mathrm{Fe}$ are presented. More information can be found in the associated Diploma thesis by Schmidt [Schmidt07]. Field ion micrographs of the composition of $\mathrm{Cu}-2.5$ at.\%Fe after 2 and $10 \mathrm{~h}$ milling time are displayed in Fig. 4.29. For samples obtained after $10 \mathrm{~h}$ of milling characteristic coherent ring patterns could be observed and the micrographs were dominated by regions of rather uniform brightness. Imaging the $\mathrm{Cu}$-rich samples, the field ion micrographs mainly were devoid of grain boundaries. Thus they allowed indexing of the crystallographic poles of the Cu-matrix as exemplarily shown in, Fig. 4.29 a). Sometimes a grain boundary was found. Though, never more then two grains could be imaged at once. This leads to an estimate of several $10 \mathrm{~nm}$ for the lower limit of the grain size in accordance with the TEM observation (cf. Fig. 4.25).

However after only $2 \mathrm{~h}$ milling time, the contrast within the matrix is very poor (shown in Fig. $4.29 \mathrm{~b}$ ) and instead, Fe-rich particles, which are found for this state, image very brightly with respect to the dim Cu-matrix. This effect is due to the higher evaporation field of Fe in comparison to $\mathrm{Cu}$, which allows distinguishing between Fe-domains and $\mathrm{Cu}$-regions. Taking advantage of this difference in contrast, the size of this Fe-rich domain can be determined to be $17 \mathrm{~nm}$. Additionally, it is likely to be coherent with the surrounding Cu-region, considering the pole directly left of this Fe-domain and its ring structure which continues from the Curegion into the Fe-domain.

After $10 \mathrm{~h}$ of milling hardly any evidence for unalloyed regions was found anymore, as can 

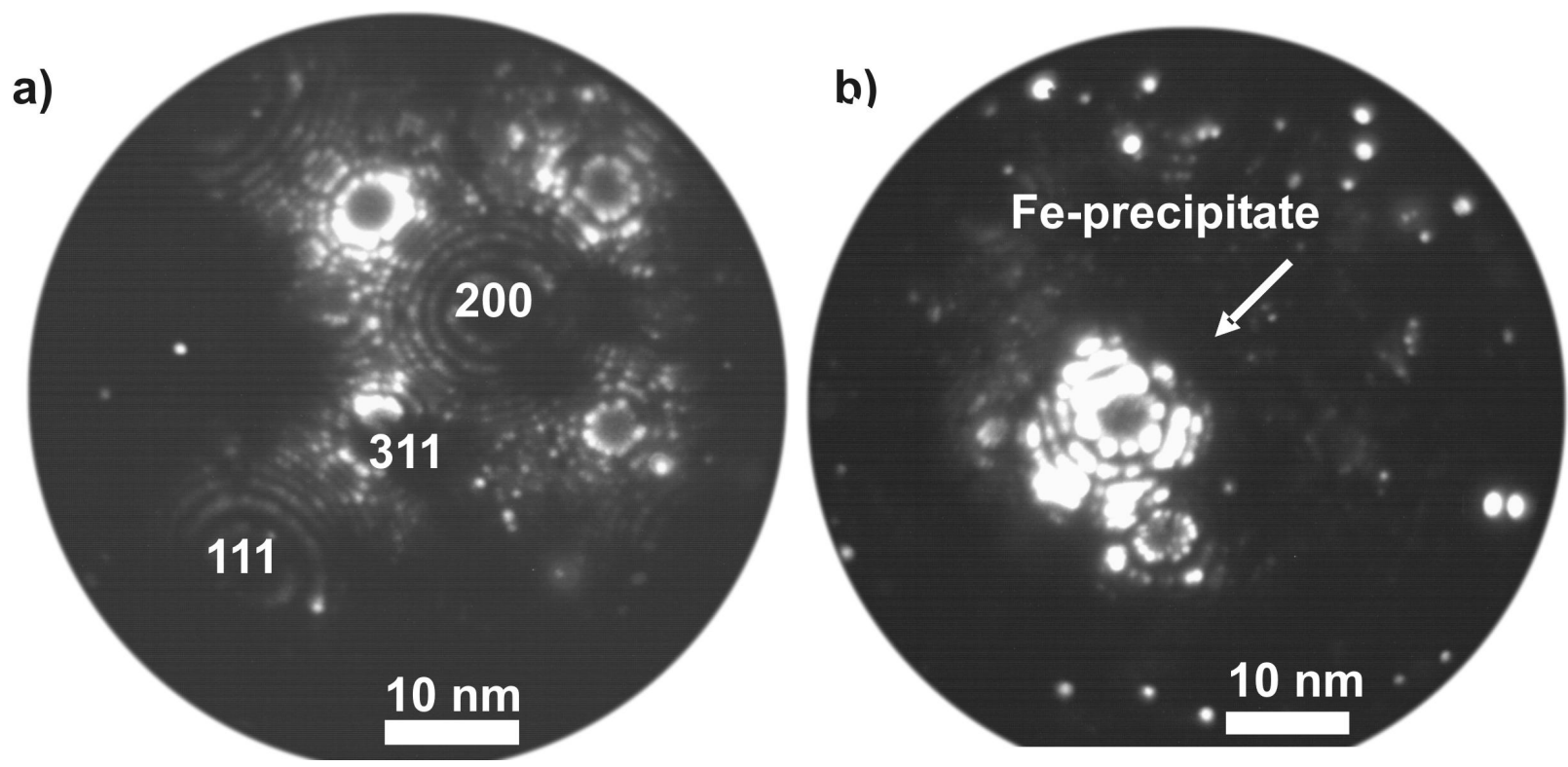

Figure 4.29: Field Ion Micrograph of Cu-2.5at.\%Fe after (a) $10 \mathrm{~h}$ and (b) $2 \mathrm{~h}$, higher evaporation field of Fe allows distinguishing between Fe-precipitate and Cu-matrix [Schmidt07]

be seen in the representative FIM image in Fig. 4.29 a).

For all milling times samples failed already during FIM analysis due to rupture. As a different crystallographic direction appeared after failure of many of the samples, the reason for this instability might be grain boundaries perpendicular to the direction of analysis. Segregation of $\mathrm{Fe}$ to the grain boundaries should increase adhesion of the grain boundaries [CAHN83]. Therefore it cannot account for the brittleness of the material. This phenomenon was largely temperature independent and occurred at temperature between 30 and $70 \mathrm{~K}$ for many measurements.

Employing Field Ion Image Tomography (FIIT) it was possible to get $3 \mathrm{~d}$ information on this Fe-domain in $\mathrm{Cu}-2.5 \mathrm{at} . \% \mathrm{Fe}$. The condition of the sample after $2 \mathrm{~h}$ milling time is imaged by means of iso brightness surfaces in Figure 4.30. The Fe-rich cluster is indicated by a white circle in the upper part of the image and a low index pole is indicated by a white arrow in the lower part. Both Fe-rich clusters and low index poles image brightly compared to the rest of the Cu-matrix. Nevertheless, they still can be distinguished as the Fe- rich regions image much brighter then even the lowest index pole in $\mathrm{Cu}$. Assuming this pole to be the prominent (110)-pole in the bcc structure, depth could be calibrated. The length of the Fe-rich region was determined to be $5.7 \mathrm{~nm}$. The width was derived from a single Field Ion Micrograph at the maximum extent to be $12 \mathrm{~nm}$, as described above.

Although the statistics of the FIM and FIIT images is not very good, the finding of a Fe- rich 


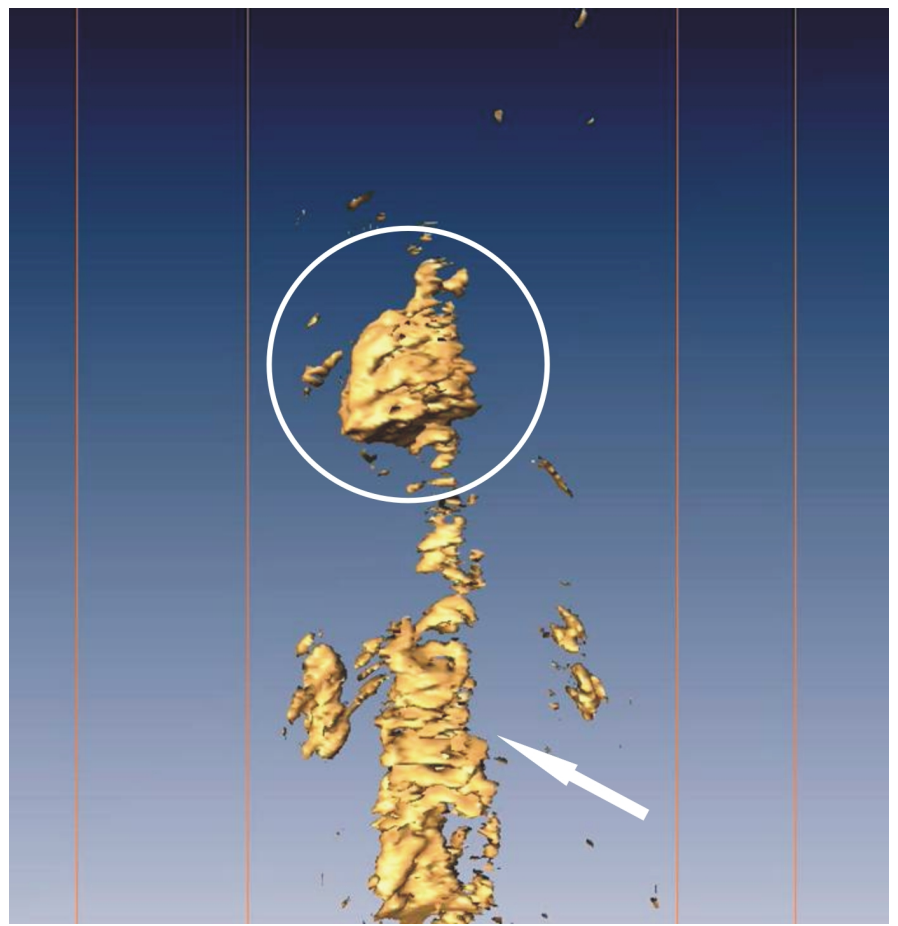

Figure 4.30: Field Ion Image Tomography: Iso brightness surfaces in Cu-2.5at.\% Fe after $2 \mathrm{~h}$ milling, Fe-rich cluster indicated by white circle, low index pole indicated by white arrow [Schmidt07]

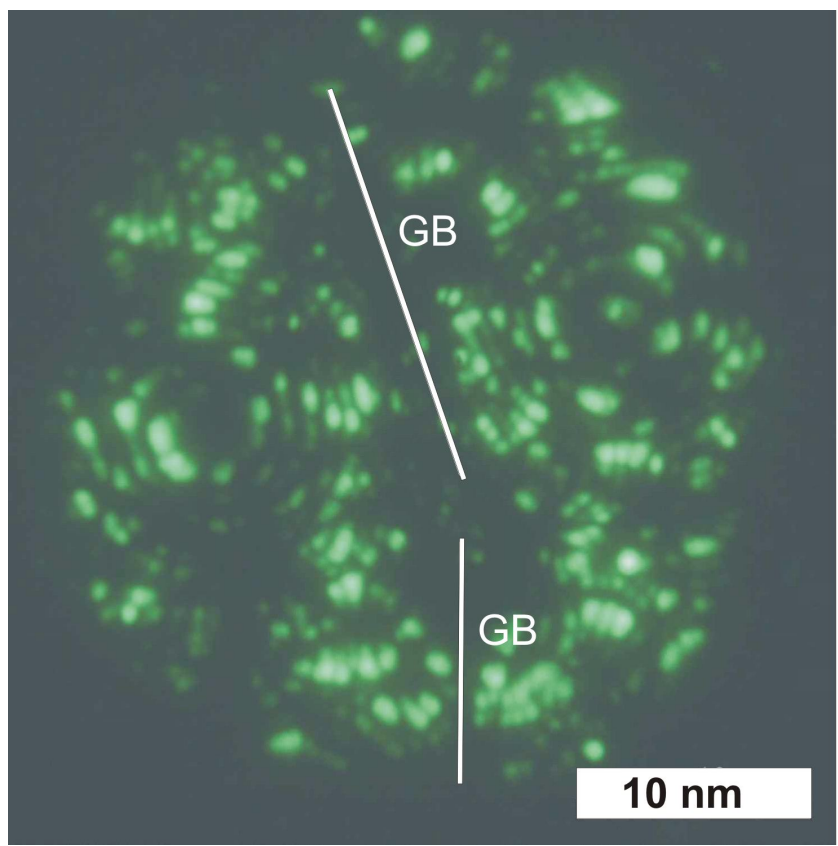

Figure 4.31: Field lon Micrograph of Fe-10at.\%Cu after $10 \mathrm{~h}$, white lines indicate grain boundaries which appear as dark lines breaking the symmetry of the concentric ring pattern 
region in the sample with lower milling time is indicative. It gives an estimate of the expected Fe- cluster size for the subsequent APT analysis.

Figure 4.31 shows a FIM image of Fe-10at.\%Cu after 10 hours milling. Large concentric ring patterns can be clearly resolved. These coherent ring patterns provide the ultimate proof for the crystallinity of the Fe-rich material. Any interruption of the strongly geometric arrangement of the ring structures is evidence for the existence of a grain boundary. As the ring structures cover the whole micrograph, no amorphous region is present and thus, this micrograph confirms the entirely nano-crystalline structure of the sample.

Exemplarily, one grain boundary (GB), appearing as a dark line in the FIM, is indicated in Fig. 4.31 by a white line as a guide for the eye. Grain boundaries were found in all FIM images taken of the Fe-rich samples. Mostly more then one GB was found per image. Thus the upper limit for the grain size can be estimated from the field ion micrographs to be a few tens of nano-metres, which is in good agreement with the findings from XRD and TEM.

\subsection{Atom Probe Tomography}

Following the structural characterisation of the powder particles, the tomographic atom probe was employed to elucidate the homogeneity of the powders on an atomic scale [Schmidt07],[Wille08],[Wille09].

In the following results for $\mathrm{Cu}-2.5$ at.\% Fe are shown first, exemplary for the $\mathrm{Cu}$-rich samples. Subsequently the Fe-rich powders are discussed more thoroughly for all investigated compositions: Fe-2.5at.\%Cu, Fe-5at.\%Cu and Fe-10at.\%Cu. These results have been obtained from analyses of the interior of the powder particles. As Oxygen is the only impurity detected with significant concentration, the distribution of Oxygen is presented in addition to the one of Copper.

As the final states of the sample preparation were performed using the FIB, the mass spectra were searched for Gallium. No peak associated with Gallium could be found.

Thus, the contamination as well as the alternation of the samples by the preparation method can be excluded.

\subsubsection{General remarks}

Before looking at the samples of different composition separately a short overview over the measured concentrations is given. 


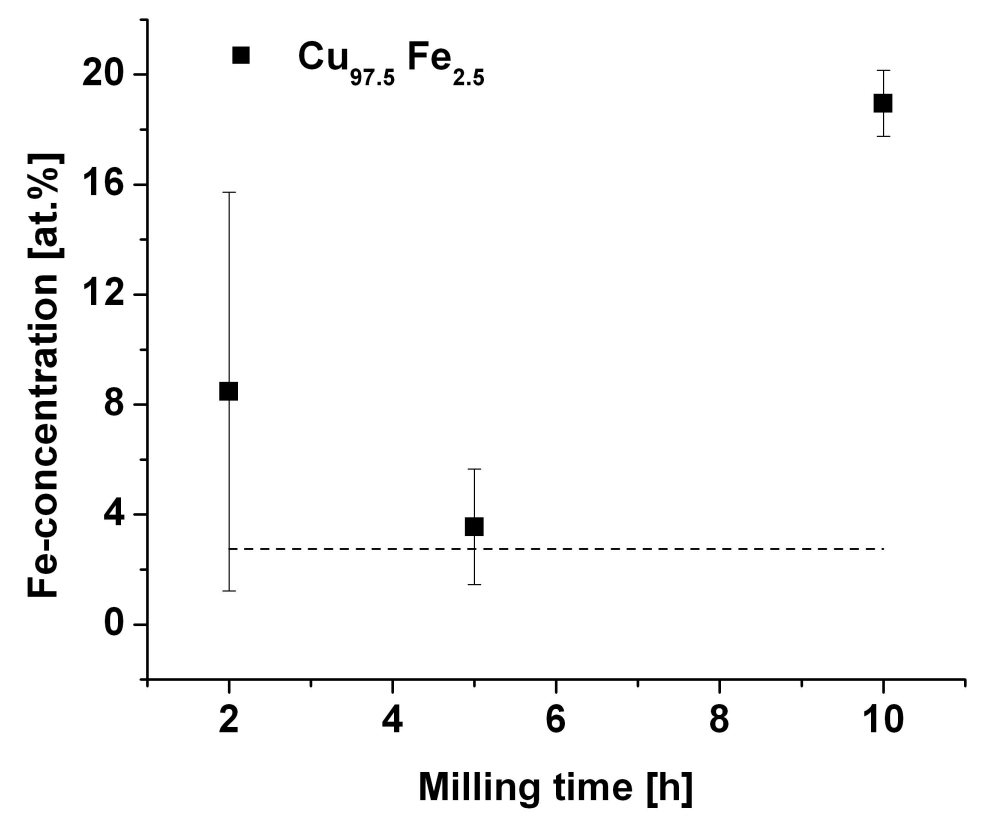

Figure 4.32: Average measured Cu-concentration versus milling time

As representative for the $\mathrm{Cu}$-rich samples, the measured average Fe-composition of $\mathrm{Cu}$ 2.5at.\% Fe is plotted versus milling time in Fig. 4.32. For all milling times the average measured composition exceeds the nominal one of 2.5at.\%. Especially after the longest milling time investigated $(10 \mathrm{~h})$ the nominal composition is not even within the error bars. Averaging over all samples of this composition the measured Fe-concentration is $(7.7 \pm 6.9)$ at.\% - three times the nominal one of 2.5at.\%. But, it still includes the nominal composition in the error bars due to the enormous variation between the individual measurements.

Generally, the mechanical stability of the Cu-rich samples might depend strongly on the Fe-contents, as Fe is supposed to increase the cohesion of grain boundaries. Thus, the atom probe specimen prepared from Fe-rich regions might be less brittle and therefore survive the cyclic deformation during atom probe analysis. None of the successfully measured specimens exhibited an Fe-concentration below the nominal composition. Most probably these specimens preferredly ruptured at the $\mathrm{Fe}-\mathrm{Cu}$ interface causing inferior mechanical properties.

Additionally, the $\mathrm{Cu}$-rich material is inhomogeneous on the $\mu \mathrm{m}$-scale as discussed in the section on light optical microscopy. As measurements only succeeded for specimens prepared from the surface of the particles, the obtained results my not be representative for the material as a whole. 


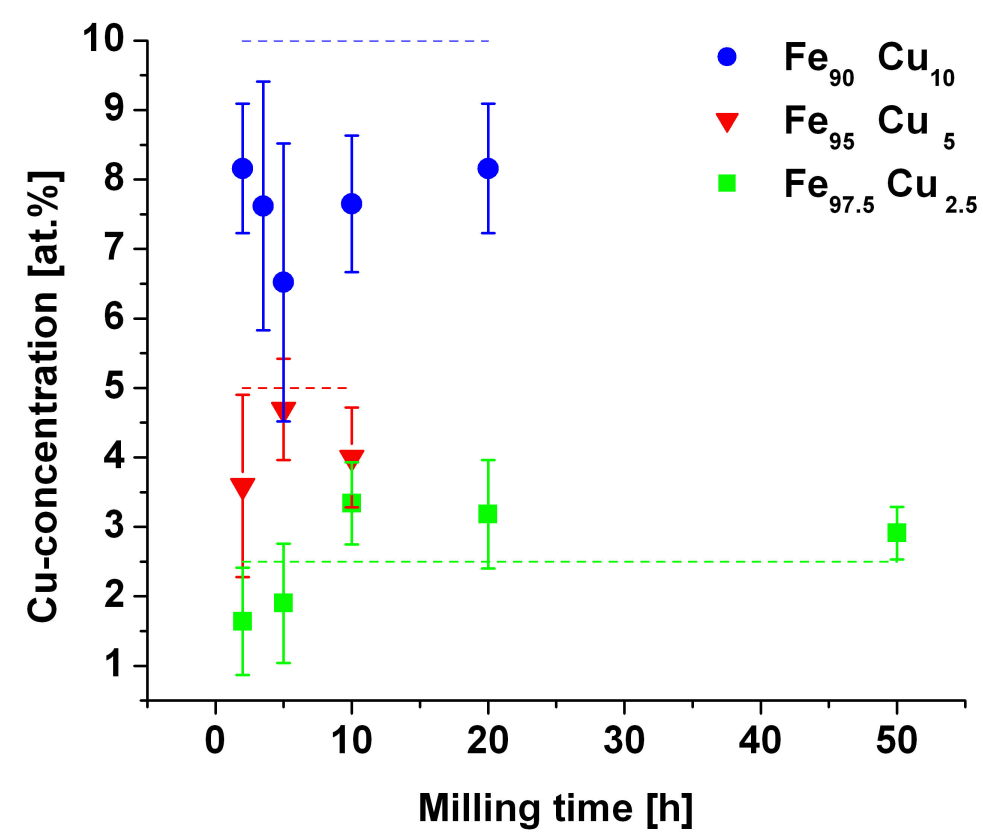

Figure 4.33: Average measured Cu-concentration versus milling time

Thirdly, during FIB preparation the sputtering of Fe-rich and Cu-rich regions was selective due to the higher sputter yield of $\mathrm{Cu}$ (sputter yield for $\mathrm{Cu}$ : 9.1; for Fe 6.9 [Orloff02]).

A similar picture arises for the Fe-rich sample with the highest $\mathrm{Cu}$-content (cf. Fig. 4.33). Again, the Fe-concentration is overestimated. The nominal composition of 10 at.\% $\mathrm{Cu}$ is not reached for any milling. It is not even included in and of the error bars. The total average of $(7.6 \pm 1.3)$ at.\%-Cu is much too low. For Fe- 5 at.\% $\mathrm{Cu}$ the lack in detected $\mathrm{Cu}$ is less pronounced. A total average of $(4.1 \pm 0.9)$ at.\%-Cu only just includes the nominal composition. For the lowest $\mathrm{Cu}$-content the total average of $(2.6 \pm 0.7)$ at.\%-Cu even slightly exceeds the nominal composition of $\mathrm{Fe}-2.5$ at.\% $\mathrm{Cu}$.

As for the Cu-rich specimens no monotonous dependence of the detected minority concentration of milling time can be found.

The general deficit of $\mathrm{Cu}$ can easily be explained consulting the XRD-spectra. The material scraped from the vessel walls showed distinct fcc $\mathrm{Cu}$ peaks. Taking into consideration the ductility of $\mathrm{Cu}$, which leads to sticking of $\mathrm{Cu}$ particles to vessel and balls, the composition of the product powder is lower than the nominal composition of the initial powder mixture.

Miller et al. [Miller03] investigated the precipitation in neutron irradiated Fe-Cu comparing SANS and APT results. As in this work, the total amount of $\mathrm{Cu}$ detected in the APT-analyses 


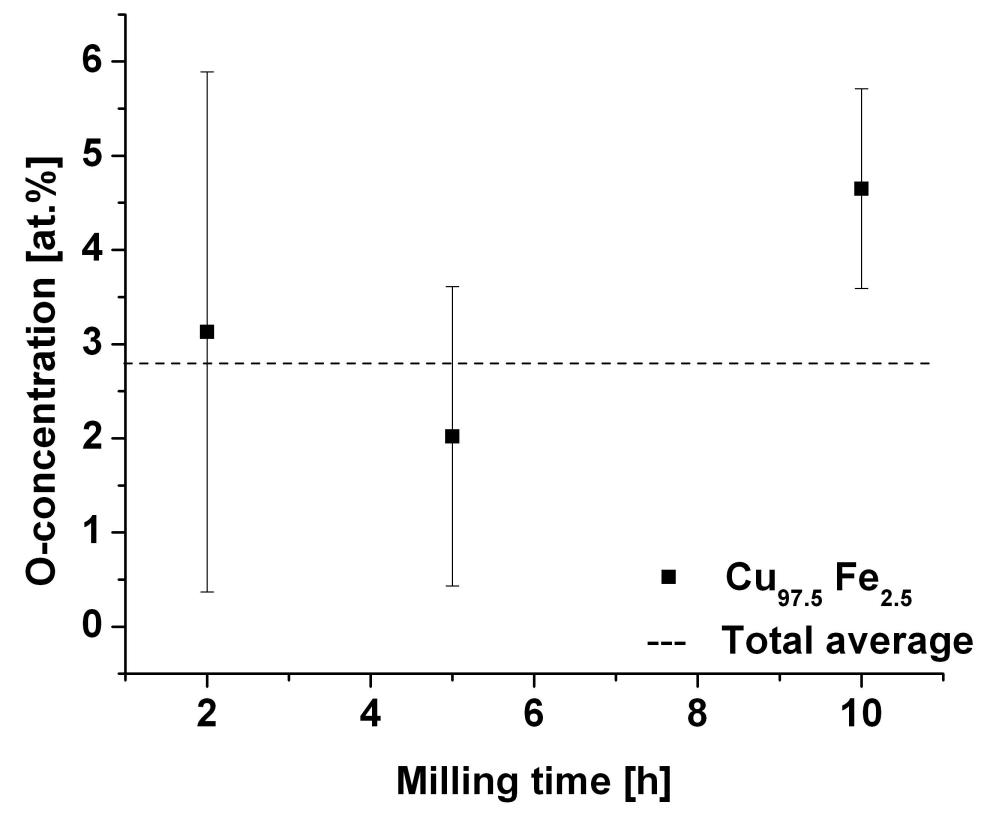

Figure 4.34: Average measured O-concentration versus milling time

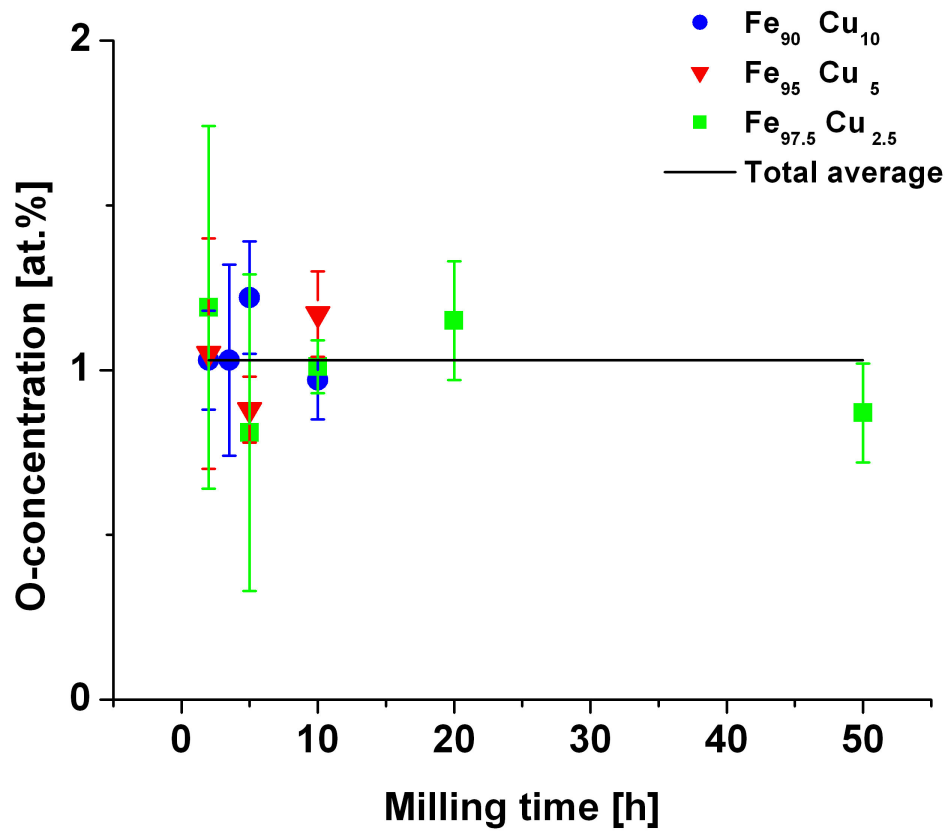

Figure 4.35: Average measured O-concentration versus milling time 
not only was lower then the nominal one ( 0.74 to 0.80 at.\%) but as well lower then in the SANS data $(0.77$ at.\%). Preferential evaporation of $\mathrm{Cu}$ is suggested to cause this lack of detecting $\mathrm{Cu}$.

For the O-content of the $\mathrm{Cu}$-rich samples only $\mathrm{Cu}-2.5$ at.\% Fe can be consulted. On the one hand, no dependence of the O-content on milling time could be found, just as for the Fecontent. On the other hand, comparing Figures 4.32 and 4.34, the average O-concentration generally increases with increasing Fe-content from below 0.5 at. $\%$ up to 6 at.\% in the individual measurements. So, the Oxygen seems to be connected to the Fe-content but unconnected to the milling time.

Contrariwise, in the Fe-rich samples the O-concentrations average approximately 1 at.\%. No dependence on either milling time or $\mathrm{Cu}$-content can be found (cf. Fig.4.35).

Especially the rather constant $\mathrm{O}$-contents of the Fe-rich samples compared to the increase of $\mathrm{O}$-concentration with increasing Fe-content in the $\mathrm{Cu}$-rich samples raises the question of the origin of this impurity. Most probably this contamination stems from the initial pure powders. The input of Oxygen during the milling process can be excluded for two reasons. First, the O-concentration does not increase with milling for any of the samples. Second, the powder of Fe-10 at.\%Cu - $10 \mathrm{~h}$ - was milled without protective Argon atmosphere. Nevertheless, the average $\mathrm{O}$-concentration is the lowest of all powders with the concentration $\mathrm{Fe}-10 \mathrm{at} . \% \mathrm{Cu}$.

Therefore, this O-contamination most probably stems from the initial pure powders. Unfortunately, since the initial powders were not available for further investigation, the question of O-contamination cannot definitely be clarified, here.

\subsubsection{Cu-rich samples: Cu-2.5at.\%Fe}

Astonishingly, the $\mathrm{Cu}$-rich samples were very brittle resulting in frequent rupture of the specimen due to the cyclic deformation by voltage pulsing. As described in the chapter on experimental methods, this specimen failure could not be avoided by variation of measurement parameters. Neither did an annealing treatment lead to an improved mechanical stability. Only the samples with the lowest Fe-concentration cut from the surface of the powder particles yielded statistically relevant data sets. All these measurements of $\mathrm{Cu}-2.5 \mathrm{at} . \%$ Fe were clearly inhomogeneous.

The inhomogeneities were investigated by concentration depth profiles. For this purpose an analysis cylinder was placed perpendicularly to the inhomogeneity. Between the Fe-rich regions the Fe-concentration usually was below the average concentration of the complete measurement, as can be seen in Fig. 4.36. Furthermore this measurement of $\mathrm{Cu}-2.5 \mathrm{at} . \% \mathrm{Fe}$ after $2 \mathrm{~h}$ milling shows two distinctly different Fe-regions. One of them is of irregular shape 


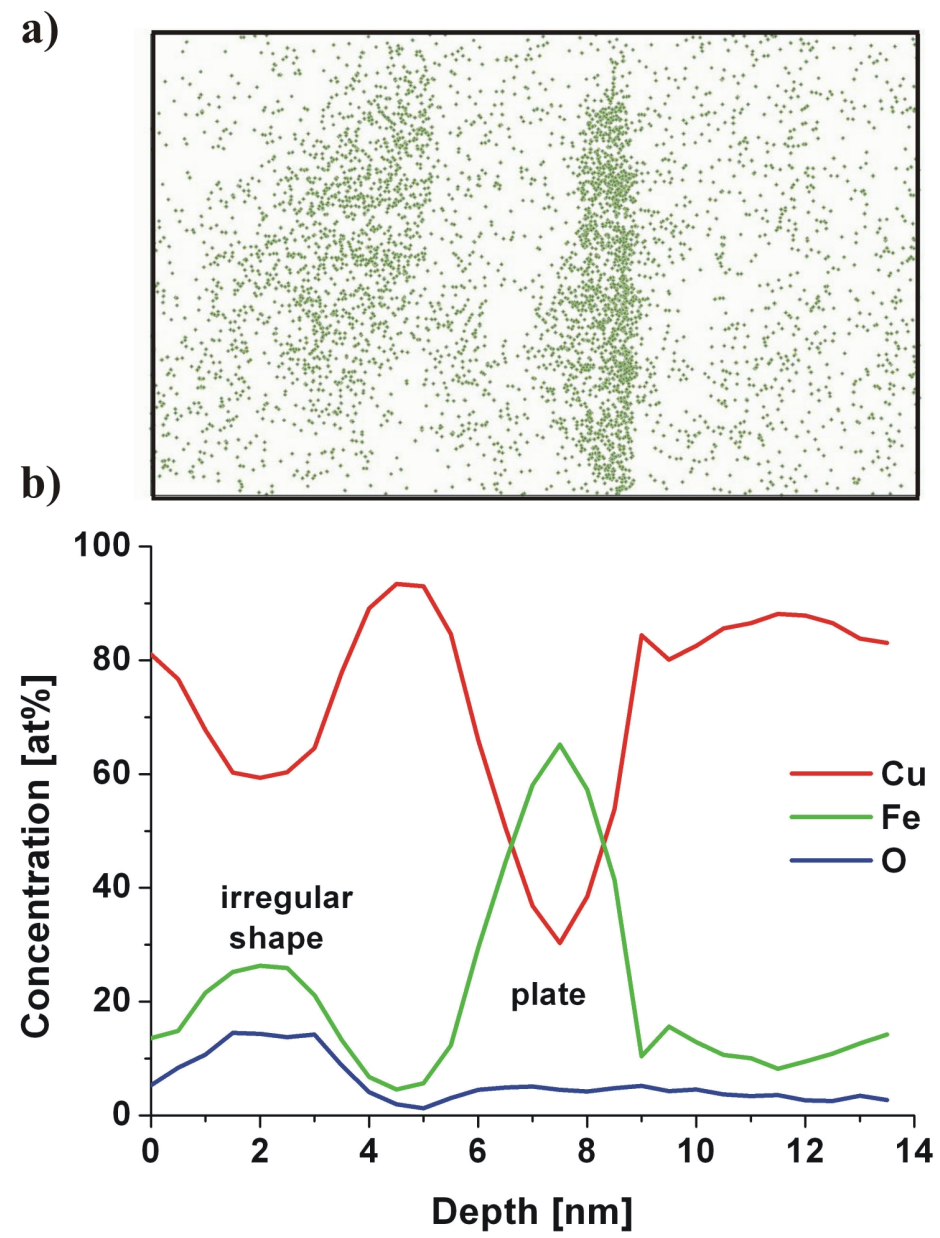

Figure 4.36: a)APT-volume of Cu-2.5at.\%Fe after $2 \mathrm{~h}$ (only Fe atoms displayed), b) corresponding concentration depth profile along the analysis cylinder showing an inhomogeneous Fe-distribution with an irregularly shaped Fe-rich region with enormous O-content(> 15 at.\%) and an Fe-rich plate with little O-contamination ( $<5$ at.\%) [Schmidt07]

and has a composition of approximately 30at.\% Fe and $>15$ at.\% Oxygen. The other one is nearly devoid of Oxygen but contains up to 70at.\% Fe.

While nearly all Fe-rich regions found contained large amounts of oxygen only one more Fe-enrichment was found without associated Oxygen.

This second O-free Fe-enrichment is shown in Fig. 4.37. As during field evaporation the imaged grain was shrinking, the Fe-enrichment in the subsequent APT measurement most probably represents a grain boundary. The thickness of this Fe-layer amounts to only $0.5 \mathrm{~nm}$, which is in the range expected for a grain boundary. Due to the higher cohesive energy of Fe compared to $\mathrm{Cu}$, a segregation of $\mathrm{Fe}$ to $\mathrm{GBs}$ of $\mathrm{Cu}$ is not expected [Eckert93b] while the opposite has frequently been observed [Cahn83]. However, in the limited amount of successful 
a)

b)

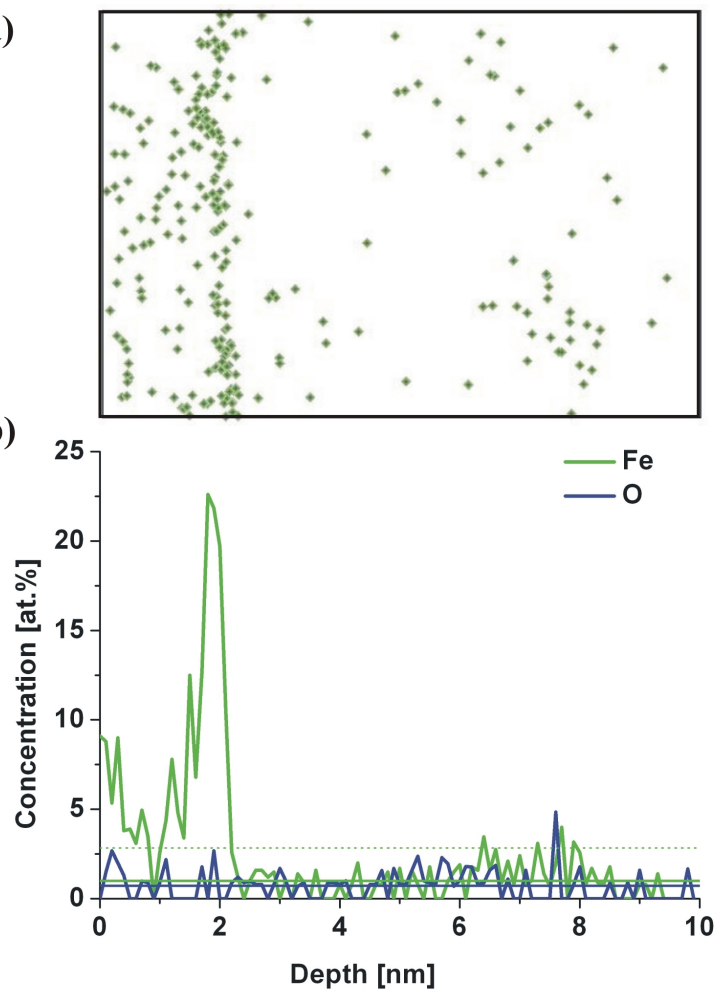

Figure 4.37: a) APT-volume of Cu-2.5at.\%Fe after $2 \mathrm{~h}$ (only Fe atoms displayed), b) corresponding concentration depth profile along the analysis cylinder showing an plate-shaped Fe-enrichment [Schmidt07]

measurements of the $\mathrm{Cu}$-rich alloys no further hint of grain boundary segregation has been found.

For longer milling times no indication for GB segregation could be found. Neither were any plate shaped Fe-enrichments detected. But, irregularly shaped Fe-enrichments were regularly present in the samples. These enrichments are usually accompanied by elevated concentrations of oxygen. Generally, the average $\mathrm{O}$-concentration in the $\mathrm{Cu}$-rich samples increases with increasing Fe-content from below 0.5 at.\% up to 6at.\%. Mostly these O-rich samples are as well Fe-rich with Fe-concentrations > 10at.\%. Both the Fe and $\mathrm{O}$ are not evenly distributed throughout the sample, but clustered.

The Fe-enrichment in Cu-2.5at.\%Fe after $10 \mathrm{~h}$, shown in Fig. 4.38, is accompanied by a significant amount of Oxygen. But, the cluster is not fully oxidised. Only the outer shell shows approximately the stochiometric ratio of 1:1 for FeO. This Fe-rich Oxide is the most probable one considering the low overall $\mathrm{O}$-concentration and consulting the Ellingham diagram.

Unfortunately, due to the poor statistics no general statement on the typical size or Ocontent of the Fe-enrichments can be made. 
a)

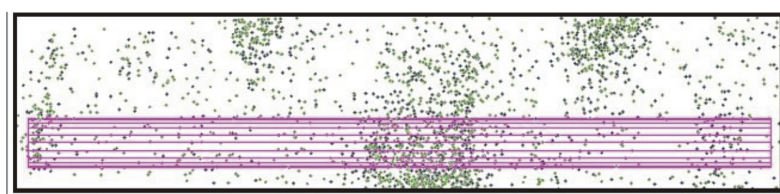

b)

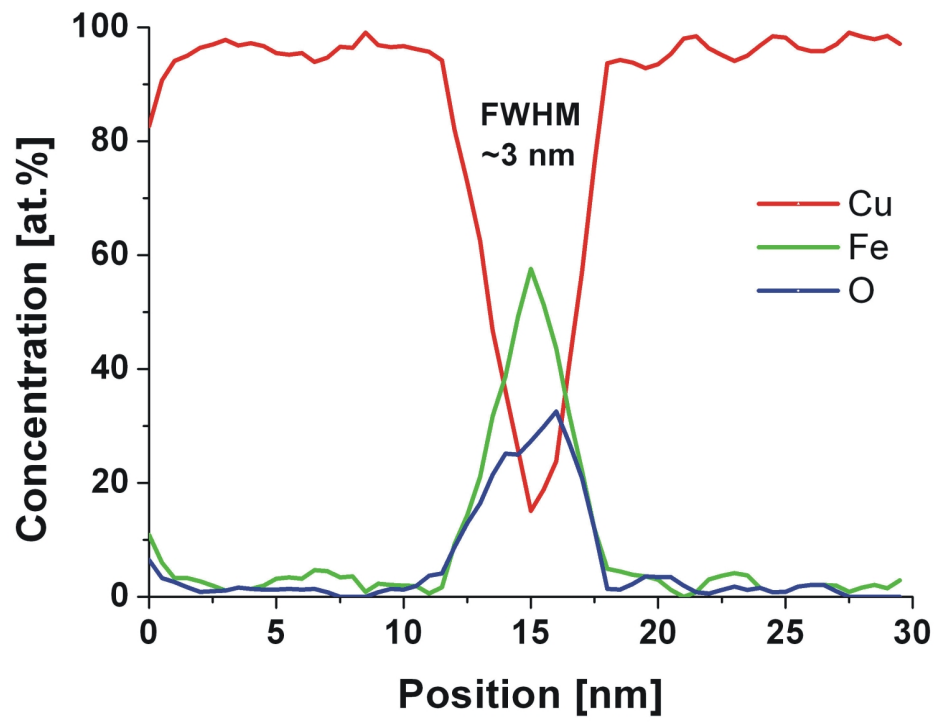

Figure 4.38: a)APT-volume of Cu-2.5at.\%Fe after $10 \mathrm{~h}$ (only Fe atoms displayed), b) corresponding concentration depth profile along the analysis cylinder showing an inhomogeneous Fedistribution with an Fe-rich cluster of about $3 \mathrm{~nm}$ [Schmidt07] 
a)
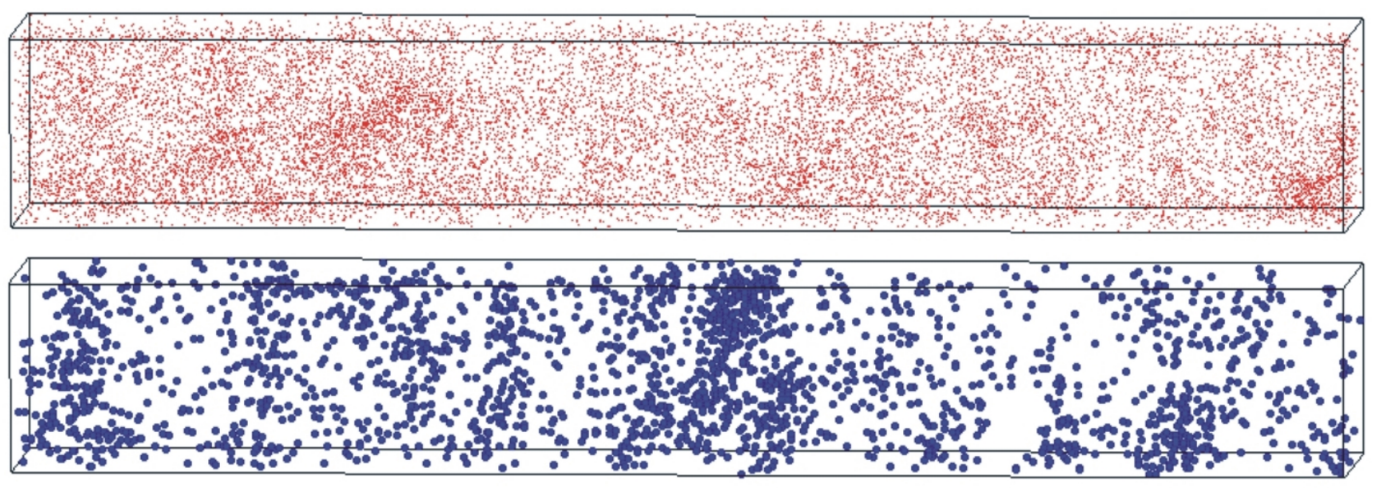

b)
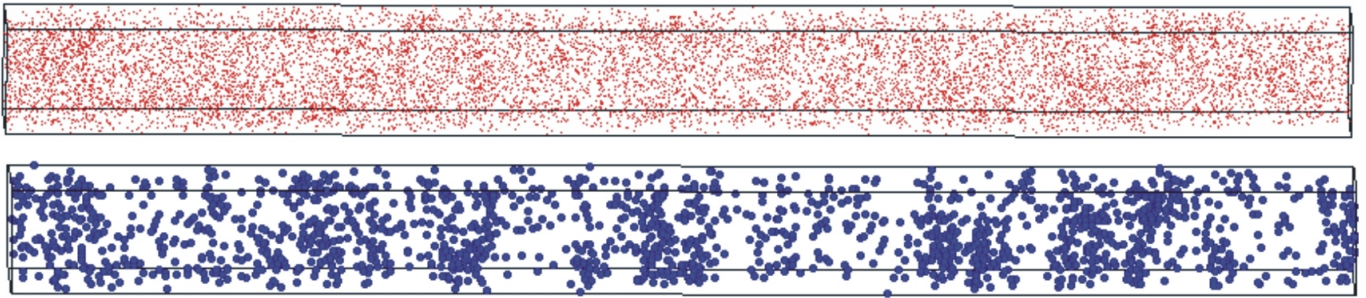

Figure 4.39: Reconstructed volume of Fe-10at.\%Cu (a)after $2 \mathrm{~h}\left(10 \times 10 \times 70 \mathrm{~nm}^{3}\right)$, b) after $10 \mathrm{~h}(15 \times 15$ $x 200 \mathrm{~nm}^{3}$ ), red: $\mathrm{Cu}$-atoms, blue: O-atoms

\subsubsection{Fe-rich samples: Fe-10at.\%Cu}

Passing on to the Fe-rich alloys, the sample of highest $\mathrm{Cu}$-content will be discussed first. Figure 4.39 shows the 3-dimensional reconstruction of two representative samples of $\mathrm{Fe}$ 10 at.\% $\mathrm{Cu}$ after $2 \mathrm{~h}$ and $10 \mathrm{~h}$, respectively. The $\mathrm{Cu}$-atoms are displayed in red and the $\mathrm{O}$ atoms blue in separate boxes.

Already in this reconstruction striking differences between the distribution of $\mathrm{Cu}$ and $\mathrm{O}$ become evident. While the clusters formed by the $\mathrm{Cu}$-atoms after $2 \mathrm{~h}$ milling time decreased in size and number when proceeding to the reconstruction of $10 \mathrm{~h}$ milling time, the clustering of O-atoms does not seem to diminish.

To quantify this first impression, concentration depth profiles are shown, which were obtained along analysis cylinders of 100-200 nm length.

In concentration depth profiles the local concentration is plotted versus depth. In this work the concentration of the minority component $(\mathrm{Cu})$ and the impurity $(\mathrm{O})$ is computed for blocks of 1000 atoms over the whole analysis volume and for blocks of 100 atoms for the selected volume of an analysis cylinder of $1 \mathrm{~nm}$ radius, respectively. These concentration values are plotted versus the block number, which corresponds to the depth. The average concentra- 
tion $c_{0}$ and confidence intervals $2 \sigma$ around the average concentration are added in the graphs. Concentration values inside these boundaries can be considered to be purely statistical with a probability of $95.5 \%$. An overview of representative APT measurements for Fe-10at.\%Cu after 2, 3.5 and $10 \mathrm{~h}$ of milling is compiled in Fig. 4.40(a)-(c).

These profile were obtained from the whole measurement of $10 \times 10 \times 70 \mathrm{~nm}^{3}$ for $2 \mathrm{~h}$ milling time and $15 \times 15 \times 200 \mathrm{~nm}^{3}$ for $10 \mathrm{~h}$ milling time. Thereby, the detected Cu-concentrations range from 6 - 8 at.\%. The deviation from the nominal composition of 10 at.\% can easily be explained consulting the XRD-spectra, as explained above. The alloy after $2 \mathrm{~h}$ milling time (Fig. 4.40 a) exhibits significant deviations from the average $\mathrm{Cu}$-concentration, frequently and strongly exceeding the limits of tolerance. The maximum deviation of 2.5 - 3 at.\% is reached several times. After $3.5 \mathrm{~h}$ time (Fig. $4.40 \mathrm{~b}$ ) the $\mathrm{Cu}$-concentration still deviates considerably from the average. However, the frequency of the biggest deviations is diminished.

After 10 h, a completely different picture arises as can be seen in Fig. 4.40 c). Hardly any more drastic deviations from the average concentration are found. Nevertheless, due to the frequent - but only slight - transgression of the $2 \sigma$-levels the material cannot be termed completely homogenous.

As already mentioned, Oxygen is the only impurity in this material detected with a significant concentration. Average O-concentrations amount to approximately 1 at.\% for all milling times. It stems most probably from the initial pure powders. In the current study, the Cu based powders seem to be covered with a thin oxide layer rather than the Fe based material, as the $\mathrm{O}$-content in the $\mathrm{Cu}$ based materials reaches values up to 6 at.\%, see above. This oxygen is distributed inhomogeneously in the material after $2 \mathrm{~h}$ as can be seen in Fig. 4.40 a). But in contrast to the behaviour of $\mathrm{Cu}$, the $\mathrm{O}$-distribution does not become homogeneous after prolonged milling, as shown in Fig. $4.40 \mathrm{~b}$ ) and c). Even after $10 \mathrm{~h}$ of milling the O-concentration is still strongly inhomogeneous. For all milling time the maximum deviation from the average $\mathrm{O}$-concentration is about 2 at.\%. The frequency of transgression does not decrease either.

Reducing the length scale once more, Fig. 4.41 shows the concentration depth profiles along a cylinder of $1 \mathrm{~nm}$ radius for the same sample as above. This plot comprises only 100 atoms per block, resulting in higher $2 \sigma$-levels. At the same time, the concentration variations are increased as well, as the averaging process is less pronounced when decreasing the sample size. Thus after $2 \mathrm{~h}$ milling time (Fig. 4.41a) ) the $\mathrm{Cu}$-concentration reaches values as high as 20 at.\%, significantly exceeding the upper $2 \sigma$-level. The increase in tolerance $2 \sigma$ is more then compensated by the increase in concentration variation. The most significant variation within the analysis cylinder reaches 5 at.\% after $2 \mathrm{~h}$ milling compared to 3 at.\% looking at the 
a)
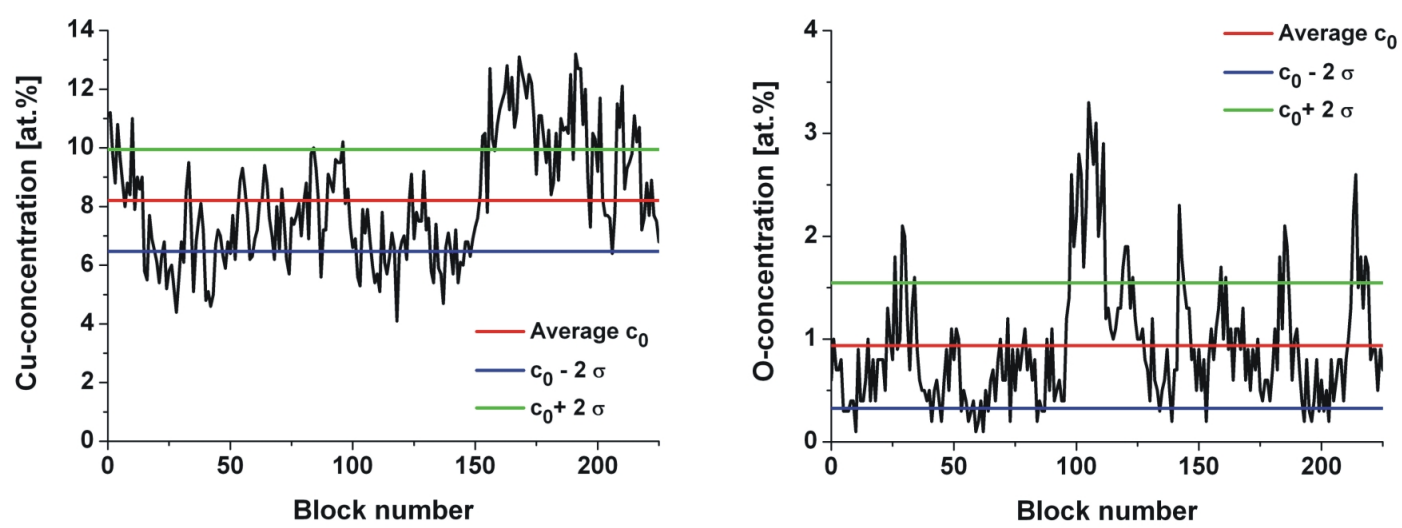

b)
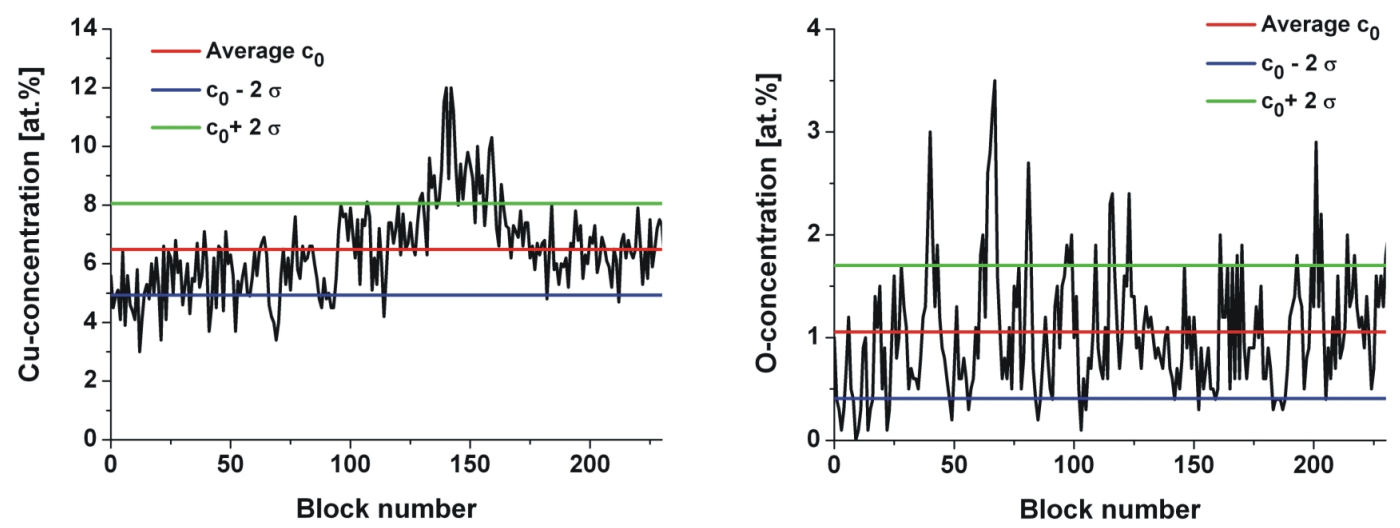

c)
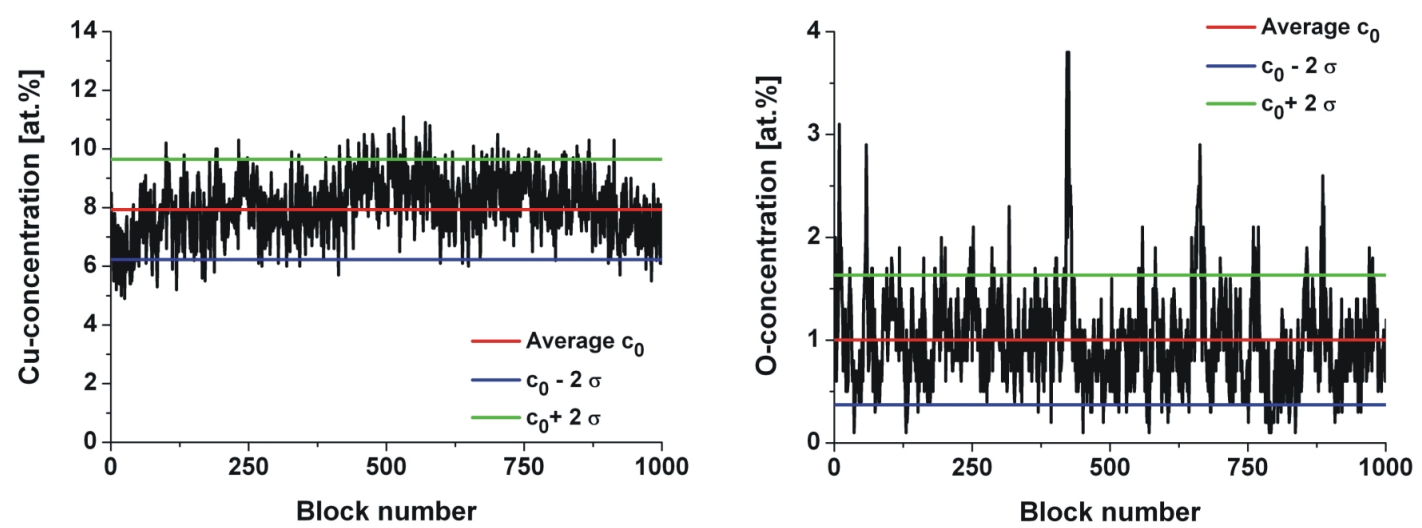

Figure 4.40: Concentration depth profiles of Fe-10at.\%Cu after a) $2 \mathrm{~h}$, b) $3.5 \mathrm{~h}$ and c) $10 \mathrm{~h}$ derived from the whole measurement, 1000 atoms per block. Inhomogeneities are drastically reduced. 
a)
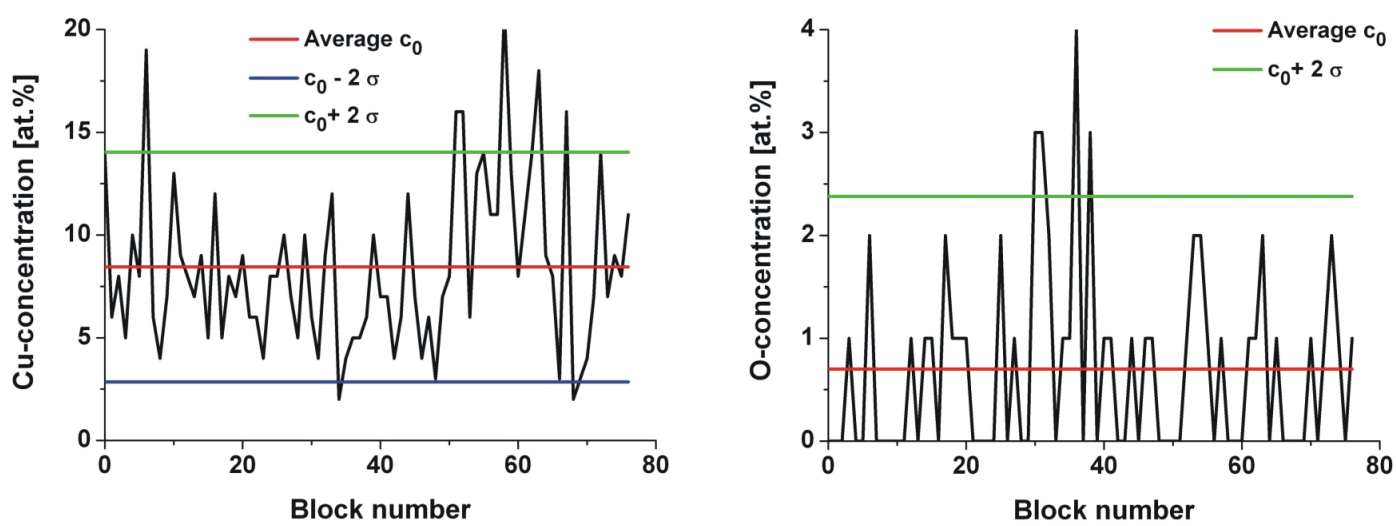

b)
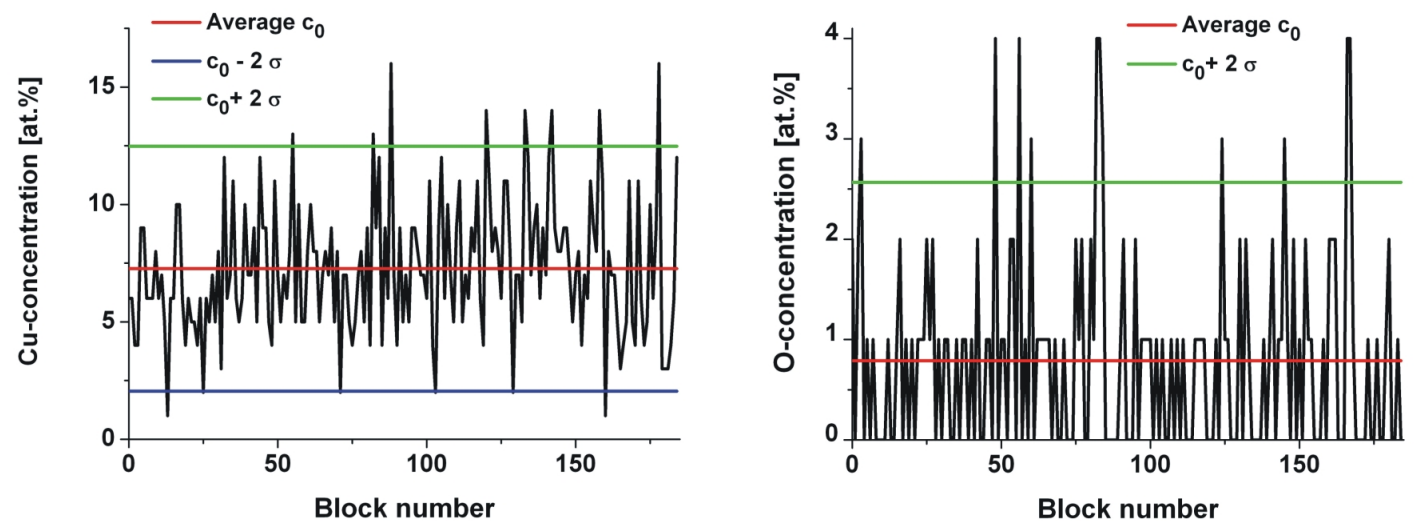

Figure 4.41: Concentration depth profiles of $\mathrm{Fe}-10 \mathrm{at} . \% \mathrm{Cu}$ after a) $2 \mathrm{~h}$ and b) $10 \mathrm{~h}$ derived from an analysis cylinder with a radius of $1 \mathrm{~nm}, 100$ atoms per block

whole volume, cf. figures 4.40 a) and 4.41 a).

The variations of the O-concentration increase as well with the $2 \sigma$-levels. But, while the $2 \sigma$-levels triple in their extent the transgressions maintain the same magnitude. The upper $2 \sigma$-levels for the coarser and the finer profile were at 0.5 at.\% and 1.5 at.\%, respectively. The concentration variations reach values of 2 at.\% and 1.5 at.\%, respectively. This indicates that the inhomogeneity is independent of the probed length scale.

Confining the probed volume for the sample after $10 \mathrm{~h}$ milling time as well to a cylinder with $1 \mathrm{~nm}$ radius, the overall impression of Fig. $4.41 \mathrm{~b}$ ) is consistent with development for the whole measurements. The profile of $\mathrm{Cu}$ is significantly smoother after $10 \mathrm{~h}$ then after $2 \mathrm{~h}$, especially the height of the concentration peaks is considerably decreased. Whereas the profile of $\mathrm{O}$ exhibits peaks of up to 4 at.\% after $10 \mathrm{~h}$ of milling, compared to a maximum height of as well 4 at.\% after $2 \mathrm{~h}$ of milling. The peaks exceeding the $2 \sigma$-levels are neither increased in magnitude nor in multitude. As opposed to the homogeneity of the $\mathrm{Cu}$, the one of $\mathrm{O}$ does 
a)

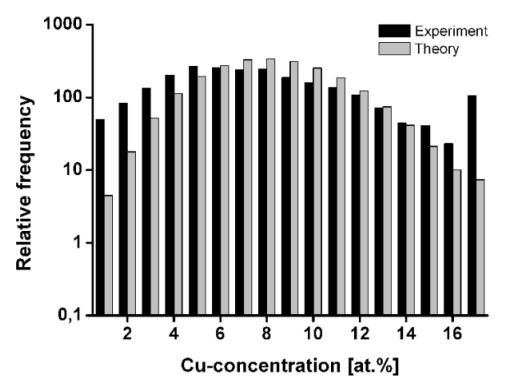

b)

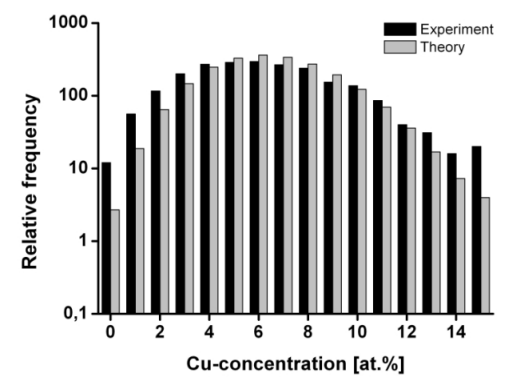

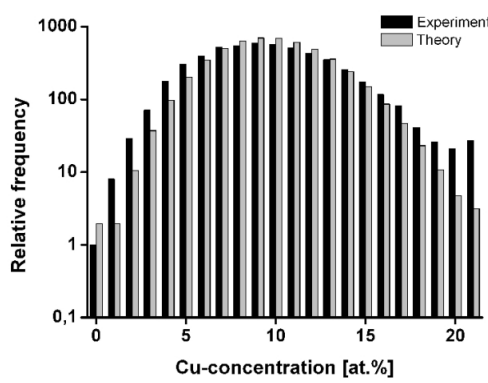

Figure 4.42: Histrograms of concentration for Fe-10at.\%Cu after a) $2 \mathrm{~h}$, b) $3.5 \mathrm{~h}$ and c) $10 \mathrm{~h}$

not seem to be enhanced by longer milling.

$\chi^{2}$-tests provide an even deeper insight into the homogeneity of the material then the concentration depth profiles. Additionally, they yield not only qualitative comparison but a quantitative one. For each treatment (composition plus milling time) the average $\chi^{2}$-value was determined from several different samples, prepared from different powder particles. The respective measurement with a $\chi^{2}$-value closest to the average value was chosen to be presented within this contribution.

The $\chi^{2}$-test, is based as well as the concentration depth profile on the concentration values obtained from blocks of 100 atoms each. A concentration frequency distribution is determined and then compared to a binomial distribution with the same average concentration as the measured one. The $\chi^{2}$-value is a measure of the deviation of the experimentally determined distribution of the elements from the binomial distribution. It indicates whether the elements in the alloy are homogeneously distributed or not. If the $\chi^{2}$-value exceeds a critical theoretical value (in this study called $\chi_{\text {theo }}^{2}(\alpha=0.05)$, which means a significance level of 95\%), it can be ascertained that the measured distribution is not homogenous - with $95 \%$ certainty. The deviation of the measured $\chi_{\exp }^{2}$ from the critical $\chi_{\text {theo }}^{2}$ is called $\alpha$. If this value is $\alpha=0.5$, the sample will be considered homogeneous.

Looking at the $\mathrm{Cu}$-concentration distributions derived by means of $\chi^{2}$-tests, the development of the homogeneity immediately becomes apparent and the assumed full homogeneity cannot be confirmed.

Figure 4.42 shows the histograms of experimental and theoretical values of the $\chi^{2}$-test. After $2 \mathrm{~h}$ of milling, the experimental $\chi^{2}$-value of the same sample of Fe-10 at.\%Cu as shown in the previous figures is as high as $\chi_{\exp }^{2}=2425$ compared to $\chi_{\text {theo }}^{2}=27.6$ leading to an alpha value of $\alpha=7.4 \cdot 10^{-132}$. Especially the deviation at very low and high concentrations contribute to the 


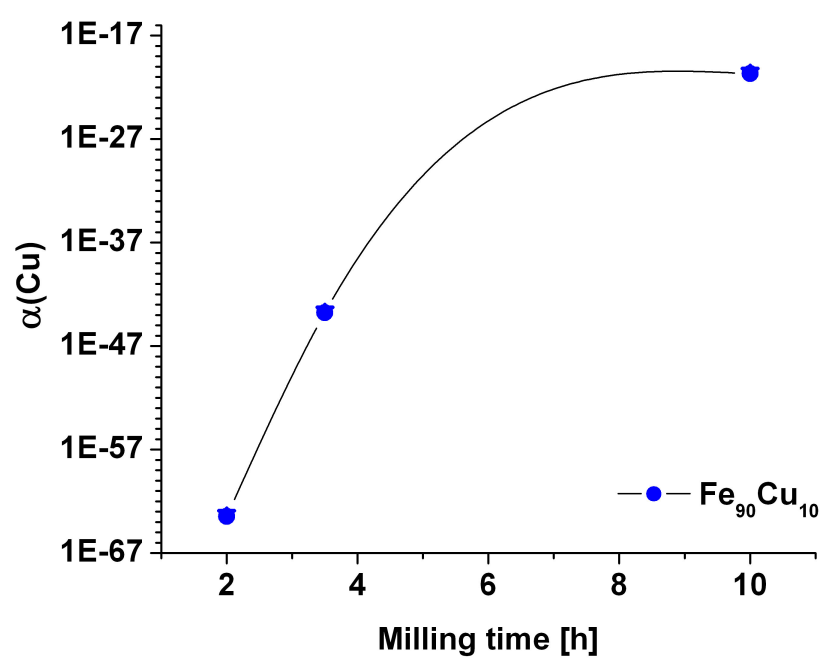

Figure 4.43: Level of significance $\alpha$ versus milling time for Fe-10at.\%Cu

high $\chi_{\exp }^{2}$-value.

Already after $3.5 \mathrm{~h}$ milling time the difference between the theoretically expected and the measured concentration distributions is clearly reduced. This results in a $\chi_{\exp }^{2}=307$ compared to $\chi_{\text {theo }}^{2}=26.3$ leading to an alpha value of $\alpha=1.0 \cdot 10^{-43}$.

After $10 \mathrm{~h}$ the deviations of the $\mathrm{Cu}$-concentration frequency distribution of $\mathrm{Fe}-10 \mathrm{at} . \% \mathrm{Cu}$ are even further reduced over the whole expected concentration range (especially for low and high concentrations). The calculated value from experimental results is $\chi_{\exp }^{2}=570$ compared to a theoretical one of $\chi_{\text {theo }}^{2}=31.4$. Although this measured value $\chi_{\exp }^{2}=570$ is in the same order of magnitude as the intermediate one after $3.5 \mathrm{~h}$ of $\chi_{\exp }^{2}=307$, the much higher sampling size in the latter sample leads to an $\alpha$ of $2.1 \cdot 10^{-21}$.

A graphical compilation of the average $\alpha$-values for Fe-10 at.\%Cu can be found in Figure 4.43. For each milling time at least 6 samples were averaged. Due to the logarithmic plot the error bars nearly vanish.

As the $\alpha$-value is a measure for homogeneity, it can be stated that the homogeneity of $\mathrm{Cu}$ in Fe-10at.\% Cu increases with milling time.

Figure 4.44 shows a prominent, since exceptionally inhomogeneous, volume of $10 \times 10 \times$ $120 \mathrm{~nm}^{3}$ of the $\mathrm{Fe}-10 \mathrm{at} . \% \mathrm{Cu}$ powder after $5 \mathrm{~h}$ milling time analysed by APT. It contains isoconcentration surfaces of 40 and 50 at.\% $\mathrm{Cu}$ in blue and red, respectively. Additionally the analysis cylinder (black) of $100 \mathrm{~nm}$ length and $1 \mathrm{~nm}$ radius is displayed. The corresponding concentration depth profile is plotted in Fig.4.44 b). 
a)

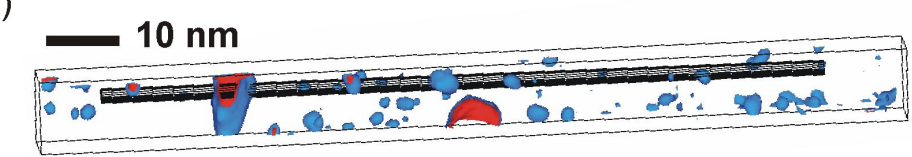

b)

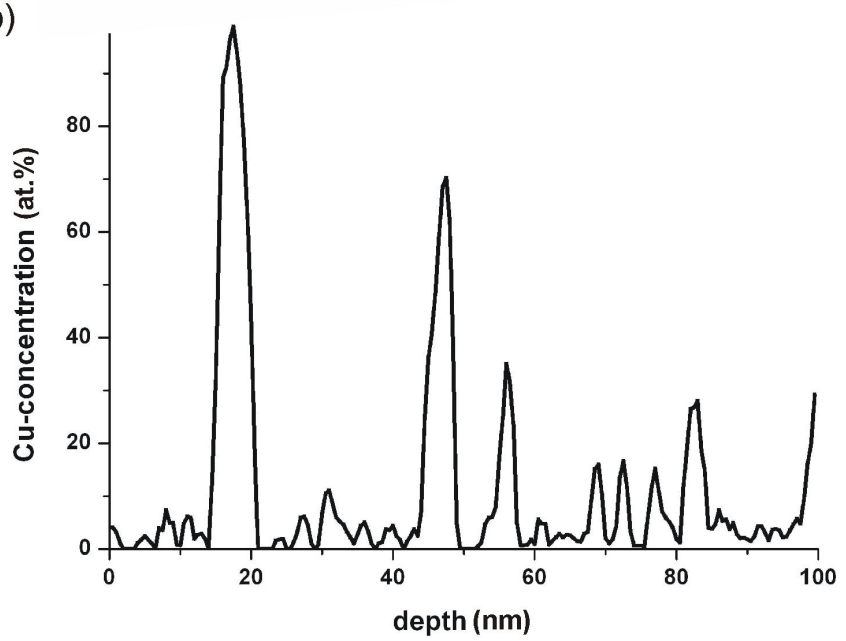

Figure 4.44: (a) APT-volume of Fe-10at.\%Cu after $5 \mathrm{~h}$ milling time including an isoconcentration surface of 50 at.\% $\mathrm{Cu}$ in red as well as an analysis cylinder. (b) The corresponding concentration depth profile along the analysis cylinder shows an inhomogeneous $\mathrm{Cu}$-distribution with a high percentage of non-alloyed $\mathrm{Cu}$.

The striking inhomogeneities reach values of up to 100 at.\% $\mathrm{Cu}$. Hence, on the nano-scale the powder particles are still composed of unalloyed $\mathrm{Cu}$ regions after $5 \mathrm{~h}$ of milling. In contrast, the XRD spectra of these samples already appeared completely alloyed after $2 \mathrm{~h}$ (cf. Fig. 4.9) as did the SAD patterns (Fig. 4.26)

\subsubsection{Fe-rich samples: Fe-5at.\%Cu}

The intermediate $\mathrm{Cu}$-concentration of $\mathrm{Fe}-5$ at.\%Cu is shown in Fig. 4.45 by means of its 3dimensional APT reconstruction. Again, the $\mathrm{Cu}$-atoms are displayed in red and the $\mathrm{O}$-atoms in blue. The first impression of the state after $2 \mathrm{~h}$ milling time $\left(15 \times 15 \times 150 \mathrm{~nm}^{3}\right)$ and the one after $10 \mathrm{~h}$ milling time $\left(16 \times 16 \times 60 \mathrm{~nm}^{3}\right)$ is drastically different. While the plate-shaped Cuenrichments visible after $2 \mathrm{~h}$ milling time reflect the lamellar microstructure that was already observed by TEM, the $\mathrm{Cu}$-distribution after $10 \mathrm{~h}$ looks rather homogeneous.

At first sight the Cu-distribution after $2 \mathrm{~h}$ milling time (Fig. $4.45 \mathrm{a}$ ) ) seems to be much more inhomogeneous then the $\mathrm{Cu}$-distribution in the corresponding state of $\mathrm{Fe}-10$ at.\% $\mathrm{Cu}$ (Fig. 4.39 a) ). This is an artifact of the recontruction. The individual samples were chosen 

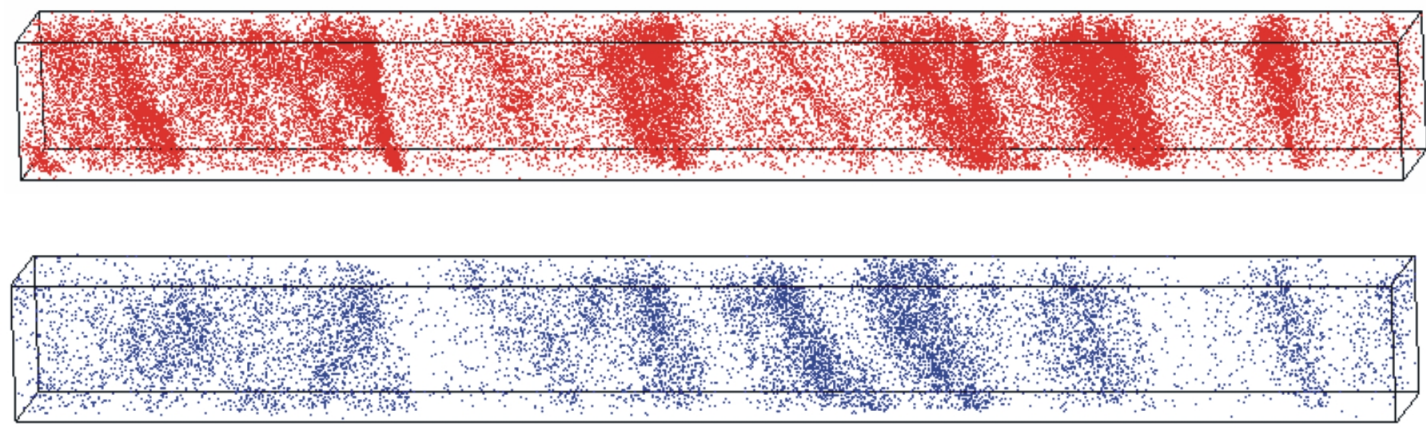

(a) After $2 \mathrm{~h}$ : clearly inhomogeneous plate-shaped distribution of both elements $\left(15 \times 15 \times 150 \mathrm{~nm}^{3}\right)$
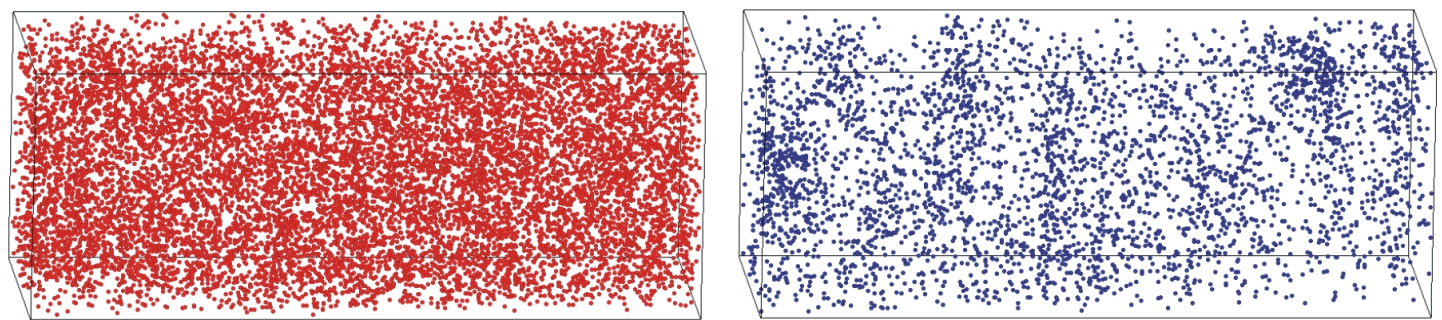

(b) After $10 \mathrm{~h}$ : increased homogeneity of both elements $\left(16 \times 16 \times 60 \mathrm{~nm}^{3}\right)$

Figure 4.45: APT reconstruction of Fe-5at.\%Cu after $2 \mathrm{~h}$ and $10 \mathrm{~h}$, red: $\mathrm{Cu}$ atoms, blue: $\mathrm{O}$ atoms

to represent the average $\alpha$-value of the respective $\mathrm{Cu}$-concentration and milling time. For both $\mathrm{Cu}$-concentration of 5 at.\% and 10 at.\% some of the individual measurements showed the distinct plate-shaped $\mathrm{Cu}$-enrichments and some did not. Additionally, these fluctuations in $\mathrm{Cu}$-density are much easier to detect in the reconstruction of $\mathrm{Fe}-5$ at.\%Cu due to the lower overall density and the resulting lower overlap.

Meanwhile, the O-distribution of Fe-5 at.\%Cu after $2 \mathrm{~h}$ looks akin to the one of $\mathrm{Fe}-10$ at.\% $\mathrm{Cu}$ in this state.

Consulting the concentration depth profiles in Fig. 4.46 a) the suspicion of a strongly inhomogeneous $\mathrm{Cu}$-distribution after $2 \mathrm{~h}$ milling time is substantiated. At an average measured $\mathrm{Cu}$-concentration of $c_{0} \approx 4$.at\% the maximum deviation from the $2 \sigma$ levels of $7-10$ at.\% is reached several times for this individual sample. For comparison, in $\mathrm{Fe}-10$ at.\%Cu the maximum deviation from the $2 \sigma$-levels only reached 3.5 at.\% at an average concentration $c_{0}$ of $>8$ at.\%.

Within the confined volume of an analysis cylinder the concentration depth profile shows the same tendency, see Fig. 4.47 a). The deviation of up to 12at.\% equals three times the average concentration. Bearing this in mind, advancing to the histogram of concentrations in Fig. 4.48 a) leads to astonishment. The histograms of concentration of Fe-10 at.\%Cu and Fe-5 
a)
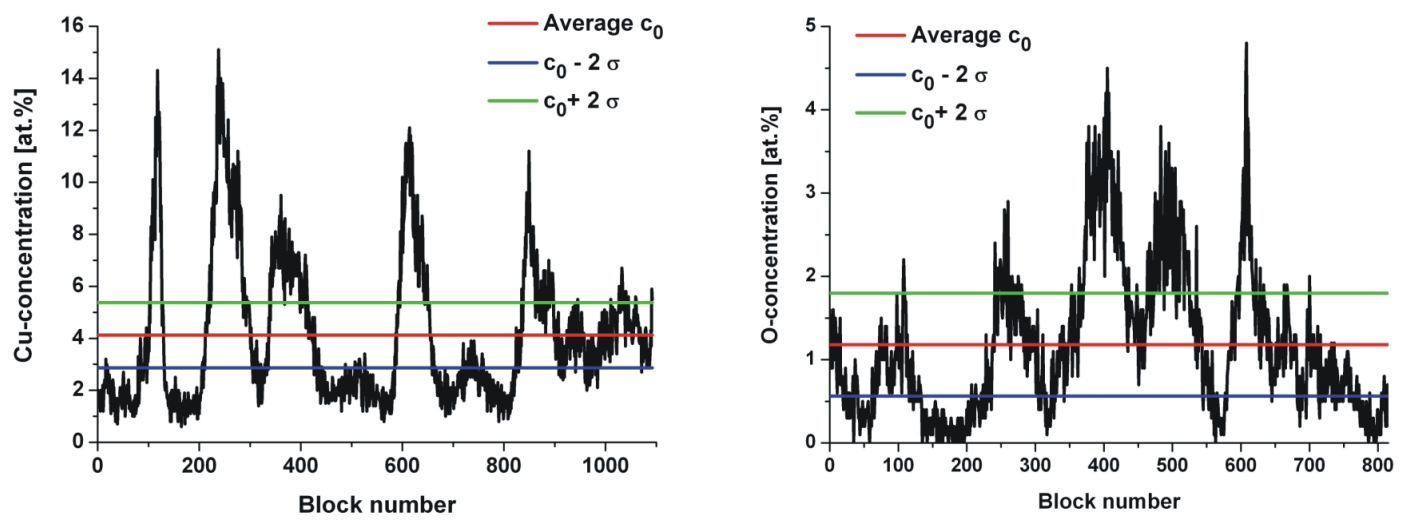

b)
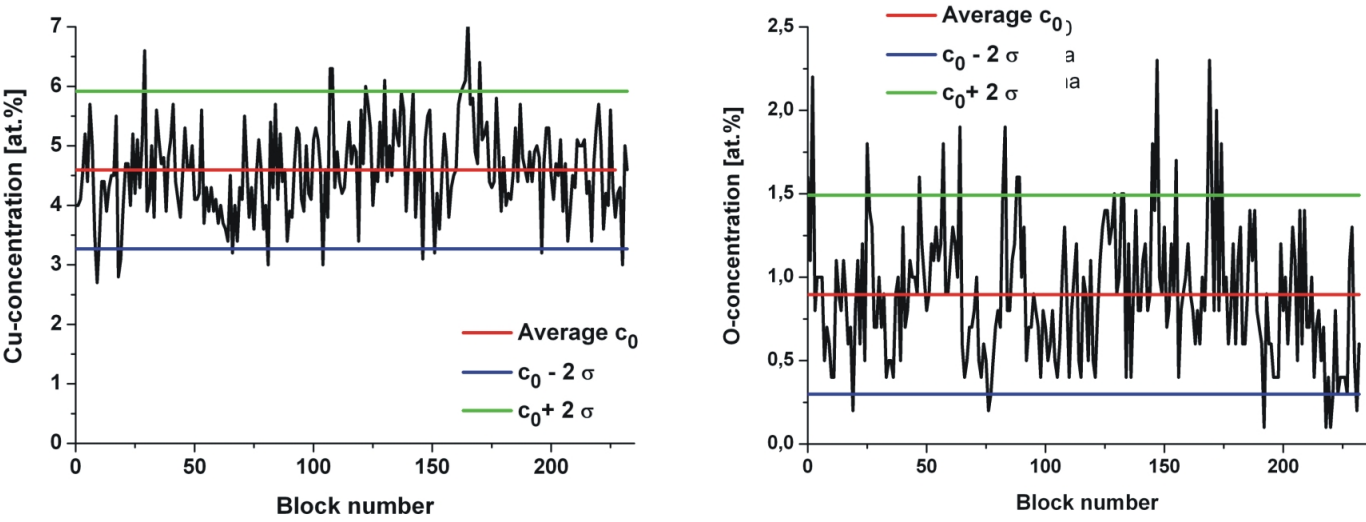

c)
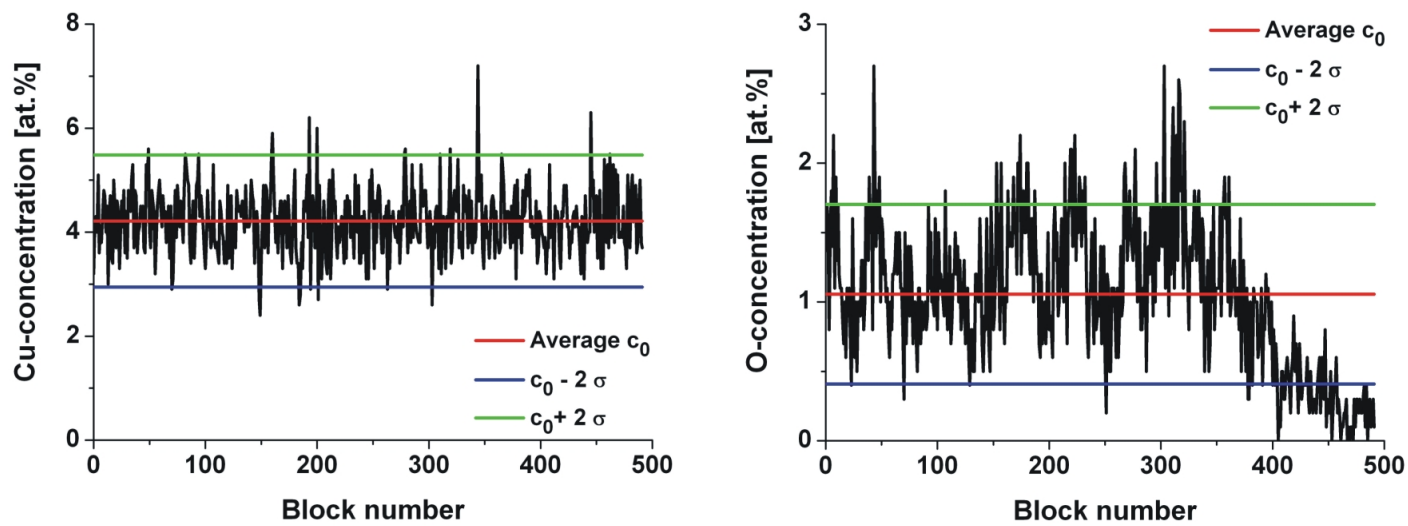

Figure 4.46: Concentration depth profiles of Fe-5at.\%Cu after a) $2 \mathrm{~h}$, b) $5 \mathrm{~h}$ and c) $10 \mathrm{~h}$ derived from the whole measurement, 1000 atoms per block. Inhomogeneities are drastically reduced. 
a)
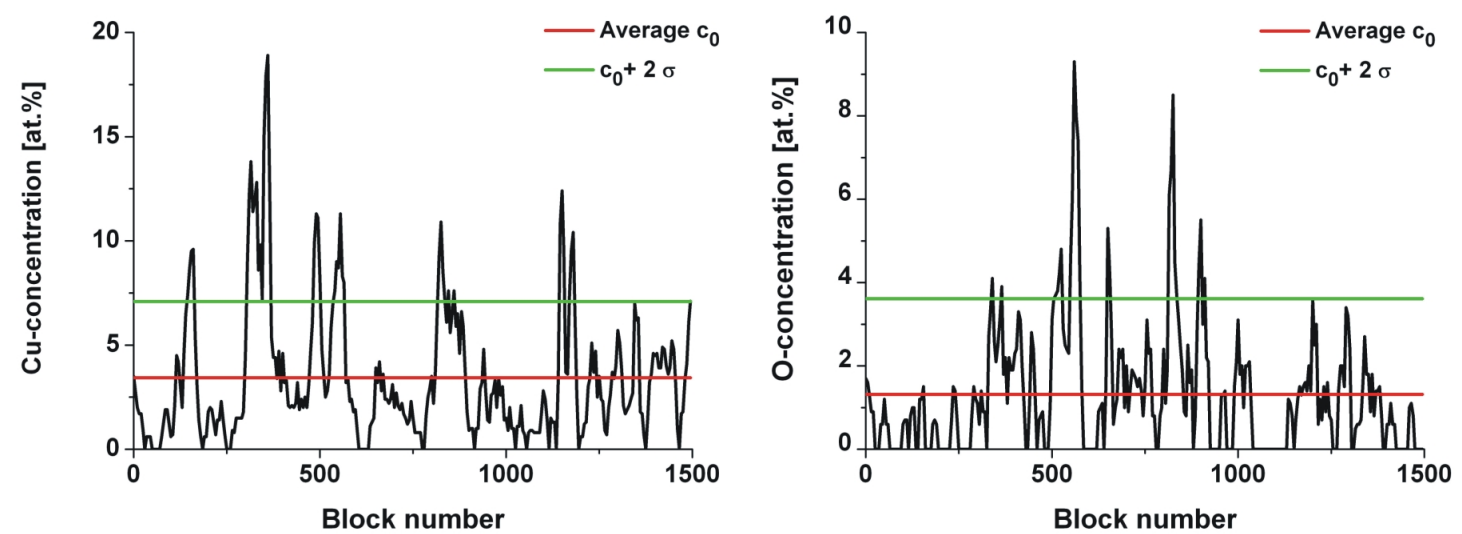

b)
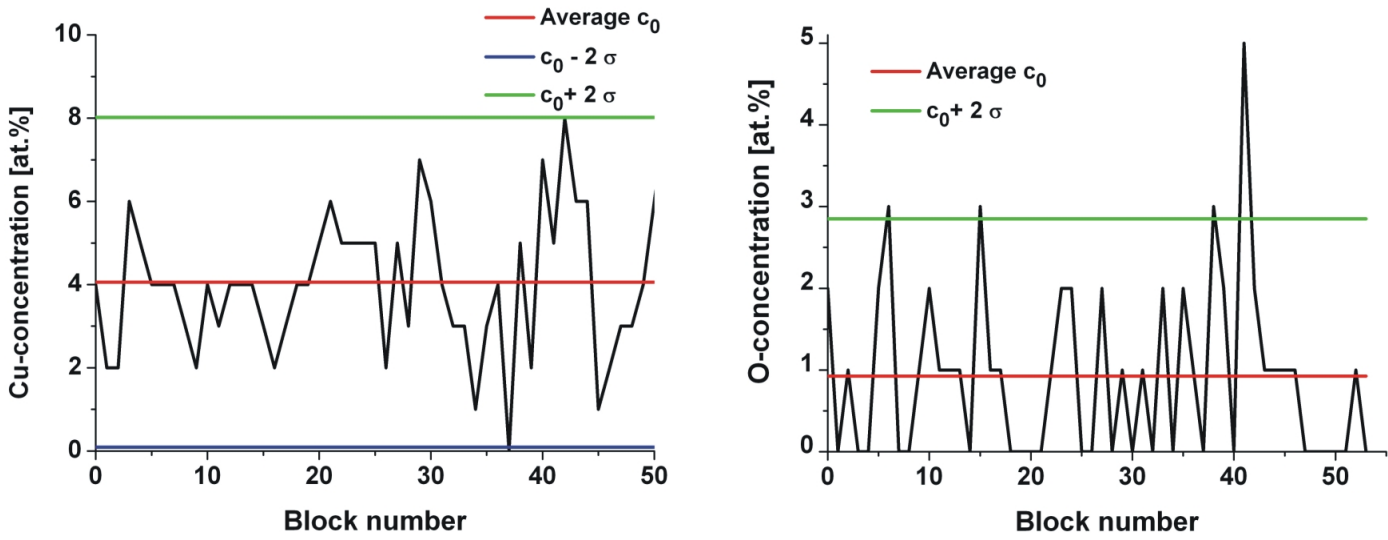

Figure 4.47: Concentration depth profiles of Fe-5at.\%Cu after a) $2 \mathrm{~h} \mathrm{,} \mathrm{b)} 5 \mathrm{~h}$ and c) $10 \mathrm{~h}$ derived from an analysis cylinder with a radius of $1 \mathrm{~nm}, 100$ atoms per block

at.\%Cu after $2 \mathrm{~h}$ milling time do not strongly differ. For both samples the main deviation from the theoretical distribution is found for the lowest and highest concentrations.

Returning to the compilation of concentration depth profiles of the whole volume in Fig. 4.46 the same trend can be observed for $\mathrm{Fe}-5$ at.\% $\mathrm{Cu}$ as for $\mathrm{Fe}-10$ at.\%Cu. The concentration profiles for $\mathrm{Cu}$ show a significant smoothing with milling time, proceeding from a) to c). With nearly the same average $\mathrm{Cu}$-concentration of $>4$ at.\% the fluctuations decrease from 10 to 1 at.\% already after $5 \mathrm{~h}$ milling time. Between $5 \mathrm{~h}$ and $10 \mathrm{~h}$ no principle change occurs in the distribution of $\mathrm{Cu}$ on the scale of the whole measurement.

For the O-distribution the statement is valid both for the concentration profile of the whole volume and those along the respective analysis cylinder. The height of the concentration fluctuation decreases with milling time when increasing it from $2 \mathrm{~h}$ to $5 \mathrm{~h}$. Between $5 \mathrm{~h}$ and $10 \mathrm{~h}$ no qualitative difference can be accounted for. 
a)

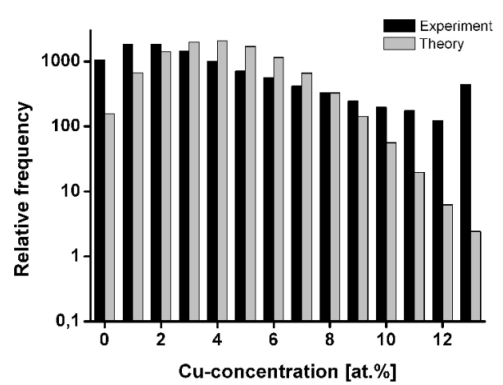

b)

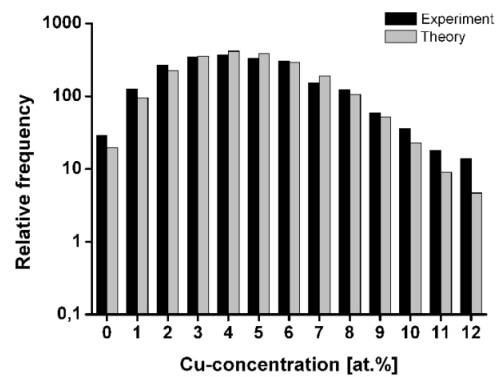

c)

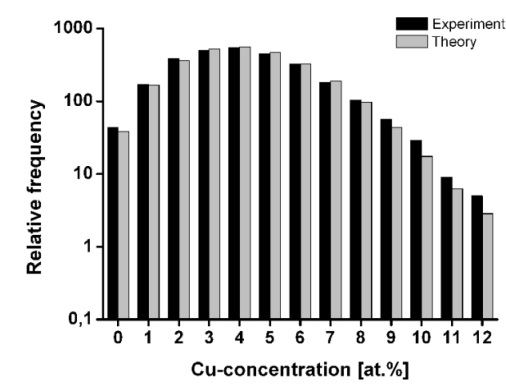

Figure 4.48: Histrograms of concentration for Fe-5at.\%Cu after a) 2 h , b) 5 h and c) $10 \mathrm{~h}$

For $\mathrm{Cu}$ as well, a drastic decrease in transgressions of the $2 \sigma$-levels is obvious in Fig. 4.47 when comparing the $\mathrm{Cu}$-concentration profiles along the respective analysis cylinder after 2 $\mathrm{h}$ and $10 \mathrm{~h}$. While the maximum difference between $2 \sigma$-level and measured concentration was 12 at.\% in Fig. 4.47 a), the concentration profile in Fig. 4.47 b) stays completely within the $2 \sigma$-levels.

This development can be followed by comparing the histograms of the measured and theoretical $\mathrm{Cu}$-concentrations in Fig. 4.48 as well. The relative frequencies of measured and theoretical $\mathrm{Cu}$-concentrations after $2 \mathrm{~h}$ (cf. Fig. 4.48a) ) diverge considerably for all concentrations but especially for the lowest and highest ones. After $5 \mathrm{~h}$ milling the divergence is less pronounced, only persisting for the borders of the measured concentration range. For the longest milling time investigated $(10 \mathrm{~h})$ the difference between theory and experiment is reduced to a minimum yielding a $\chi_{\exp }^{2}=18.8$ compared to $\chi_{\text {theo }}^{2}=22.4$. In contrast to the finding for Fe-10 at.\%Cu after the same milling time $\left(\chi_{\exp }^{2}=570\right.$ compared to $\left.\chi_{\text {theo }}^{2}=31.4 ; \alpha=2.1 \cdot 10^{-21}\right)$ the $\mathrm{Cu}$-distribution of this measurement of Fe-5 at.\%Cu after $10 \mathrm{~h}$ milling time is homogeneous and representative for this state.

The resulting average $\alpha$-value for each state is plotted versus milling time in Fig. 4.49. As already seen for $\mathrm{Fe}-10$ at.\% $\mathrm{Cu}$, the homogeneity of $\mathrm{Cu}$ increases with milling time. But, for this lower concentration of 5 at.\% a milling time of only $10 \mathrm{~h}$ suffices to produce samples which can be termed homogeneous under the assumed level o significance $(\alpha=0.05)$.

Under the hypothesis of homogeneity the probability to obtain extremer values than the observed ones is 0.09 . Thus the hypotheses is rejected at level $\alpha=0.1$ whereas it is not rejected at level $\alpha=0.05$. So the difference between the the theoretical and the experimental values cannot be regarded as significant. 


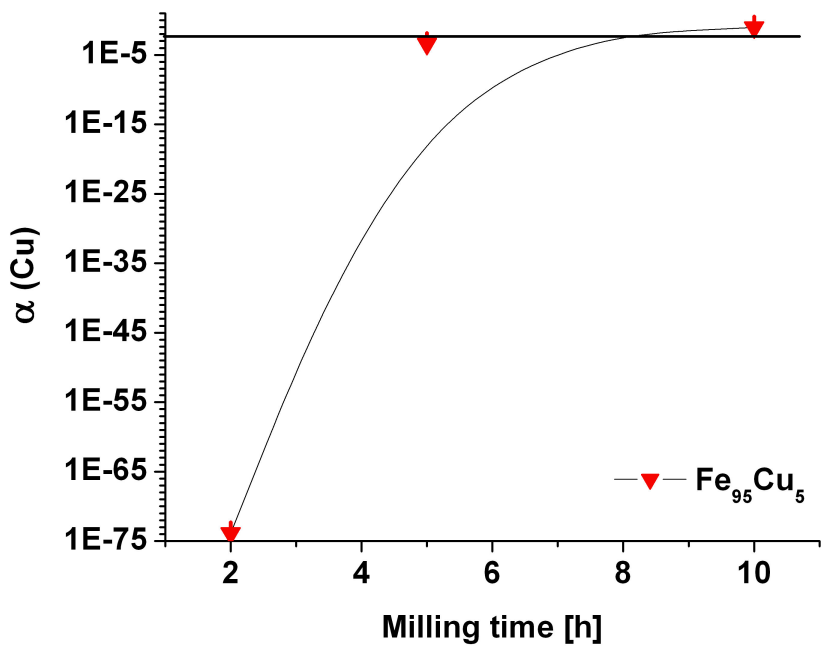

Figure 4.49: Level of significance $\alpha$ versus milling time for Fe-5at.\%Cu

\subsubsection{Fe-rich samples: Fe-2.5at.\%Cu}

Examining the Fe-rich sample with lowest $\mathrm{Cu}$-content of 2.5 at.\% the striking difference to the samples with highest $\mathrm{Cu}$-contents becomes evident already in the 3-dimensional reconstruction, as can be seen in Fig. 4.50. Due to the low $\mathrm{Cu}$-content the clearly inhomogeneous $\mathrm{Cu}$-distribution in $\mathrm{Fe}-2.5 \mathrm{at} \% \mathrm{Cu}$ can easily be observed with the naked eye, as it was the case for $\mathrm{Fe}-5 \mathrm{at} . \% \mathrm{Cu}$. This was not possible for the samples with highest $\mathrm{Cu}$-contents partly due to overlap in the 3-deimensional recontruction. As before the O-distribution is considerably inhomogeneous as well.

The arrangements of both elements reflect the findings of the TEM-investigations on the inner part of the powder particle. The elongated lamellar structure of the grains in the TEMimages is consistent with the plate-like enrichment of $\mathrm{Cu}$ and $\mathrm{O}$. The $\mathrm{Cu}$-distribution is homogenised completely after $50 \mathrm{~h}$ milling time as far as can be judged from the reconstruction, see Fig. 4.50 b). In contrast to this finding, the O-distribution does not smoothen out although the samples were milled for this prolonged time. The 3 dimensional reconstruction directly shows O-rich areas and high density fluctuations.

In the following the concentration depth profiles obtained from these volumes will be discussed. Although it is the lowest $\mathrm{Cu}$-concentration investigated, the $\mathrm{Fe}-2.5$ at.\% $\mathrm{Cu}$ material is still highly supersaturated as $\mathrm{Cu}$ has virtually no solubility within Fe. So, for the low milling time of $2 \mathrm{~h}$ the concentration depth profiles of $\mathrm{Cu}$ and $\mathrm{O}$, in Fig. $4.51 \mathrm{a}$ ), are in qualitative accordance with those of the $\mathrm{Fe}-10$ at.\% $\mathrm{Cu}$ and $\mathrm{Fe}-5$ at.\% $\mathrm{Cu}$ for the same time as expected. The 
a)
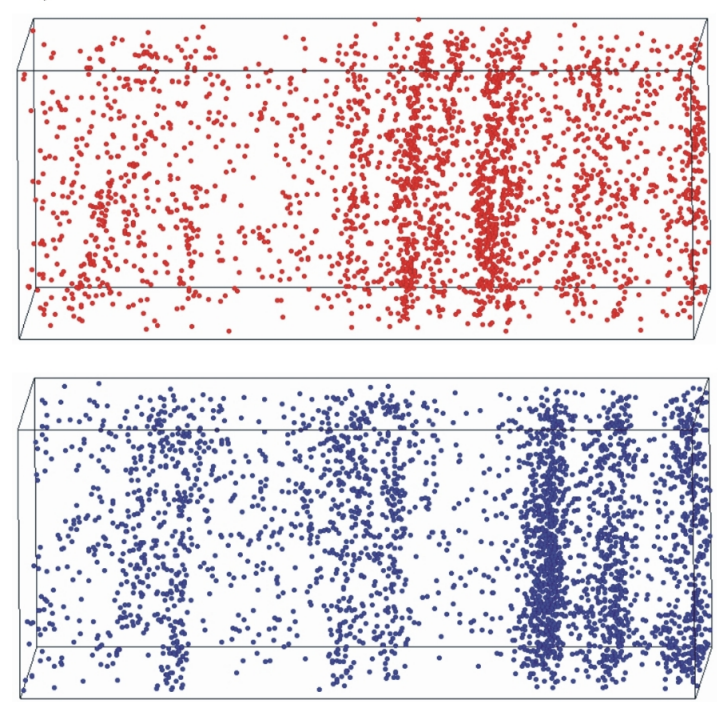

b)
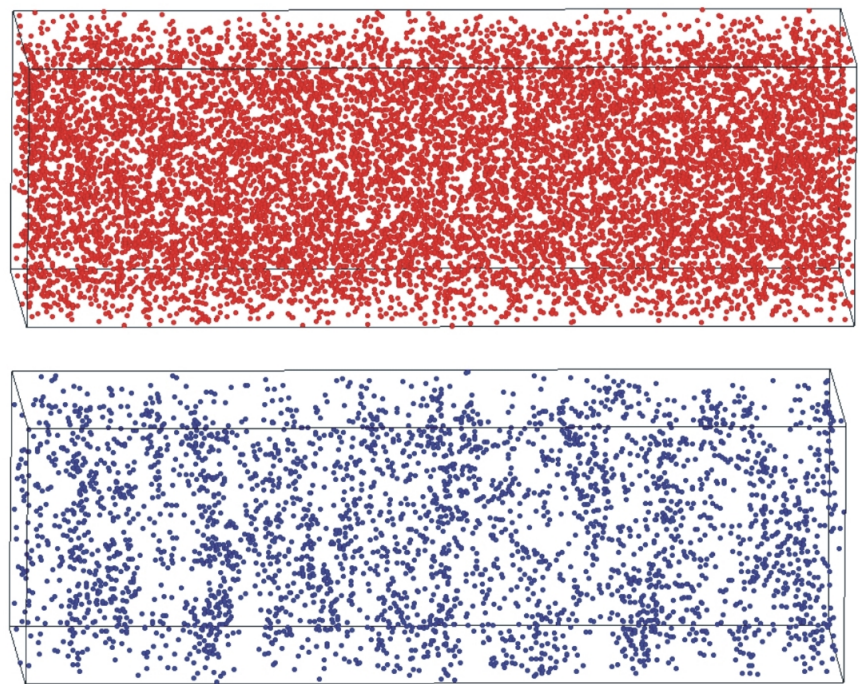

Figure 4.50: a) Reconstructed volume of Fe-2.5at.\%Cu after $2 \mathrm{~h}$, b) after $50 \mathrm{~h}$, red: Cu atoms, blue: $\mathrm{O}$ atoms

confidence intervals $2 \sigma$ are exceeded frequently and highly. The maximum deviation of the $\mathrm{Cu}$-concentration from the $2 \sigma$-level reaches 2.5 at.\% compared to 3 at.\% in Fe-10 at.\%Cu and 7 to 10 at.\% in $\mathrm{Fe}-5$ at.\%Cu.

Looking at the concentration depth profile of Oxygen, the fluctuation are as significant as in the other Fe-rich alloys. After $10 \mathrm{~h}$ milling time the transgressions of the $2 \sigma$-levels are minimal, see Fig. $4.51 \mathrm{~b}$ ). As well to higher as to lower concentration the transgressions do not exceed 0.5 at.\% , compared to 1.5 at.\% in Fe-10 at.\%Cu and 1 at.\% in $\mathrm{Fe}-5$ at.\%Cu.

The longest milling time for the material of lowest $\mathrm{Cu}$-content was $50 \mathrm{~h}$ resulting in a $\mathrm{Cu}$ concentration profile without strong alternations, see Fig. $4.51 \mathrm{c}$ ). Only few peaks slightly exceed the $2 \sigma$-levels. The highest deviations of less then 0.5 at.\% are in the same range as after $10 \mathrm{~h}$ milling time.

Comparing the development of the $\mathrm{O}$-concentration profiles with milling time a slight trend towards a smoothing of the profile can be observed for the three selected samples. However, this trend is not representative for the development of the average homogeneity of Oxygen. The samples have been selected to represent the average $\mathrm{Cu}$-distribution which did not necessarily mean they represent the average O-distribution as well. Efforts to correlate the homogeneity of $\mathrm{Cu}$ with the one of $\mathrm{O}$ failed owing to the limited investigate dvolume. The two $\alpha$-values are uncorrelated.

Within the confined volume of an analysis cylinder (radius $=1 \mathrm{~nm}$ ) hardly any difference 
a)
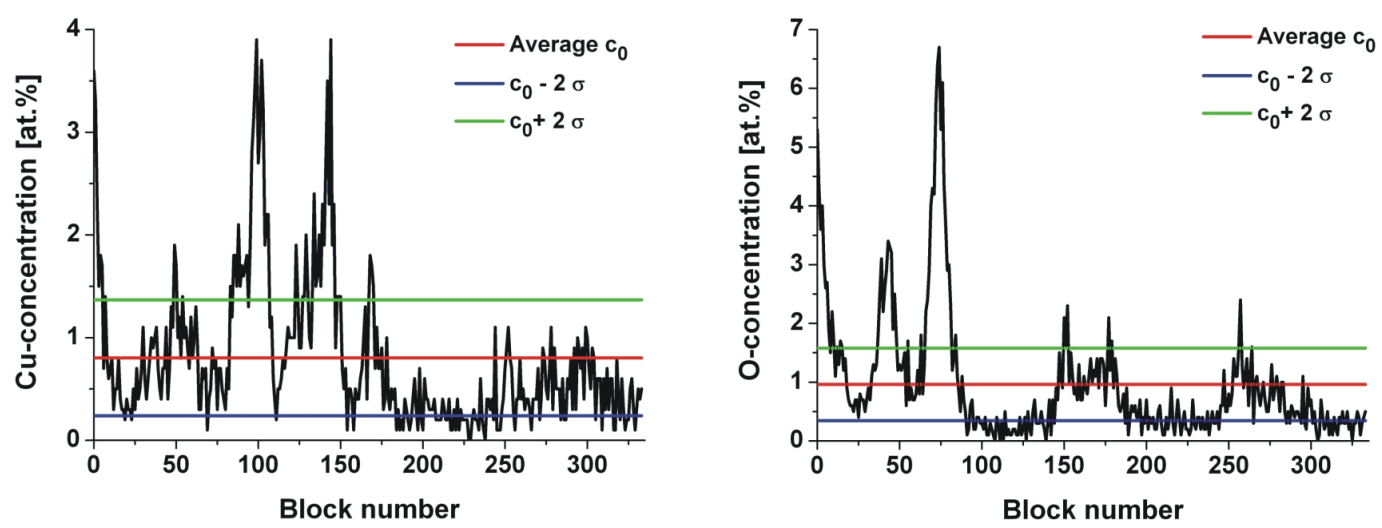

b)
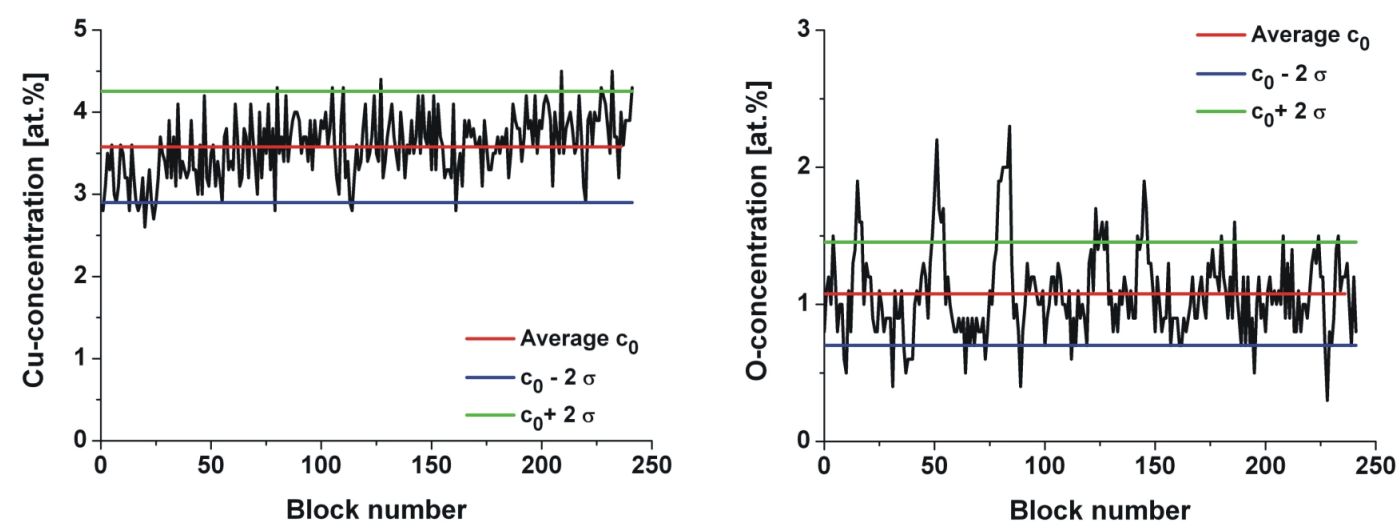

c)
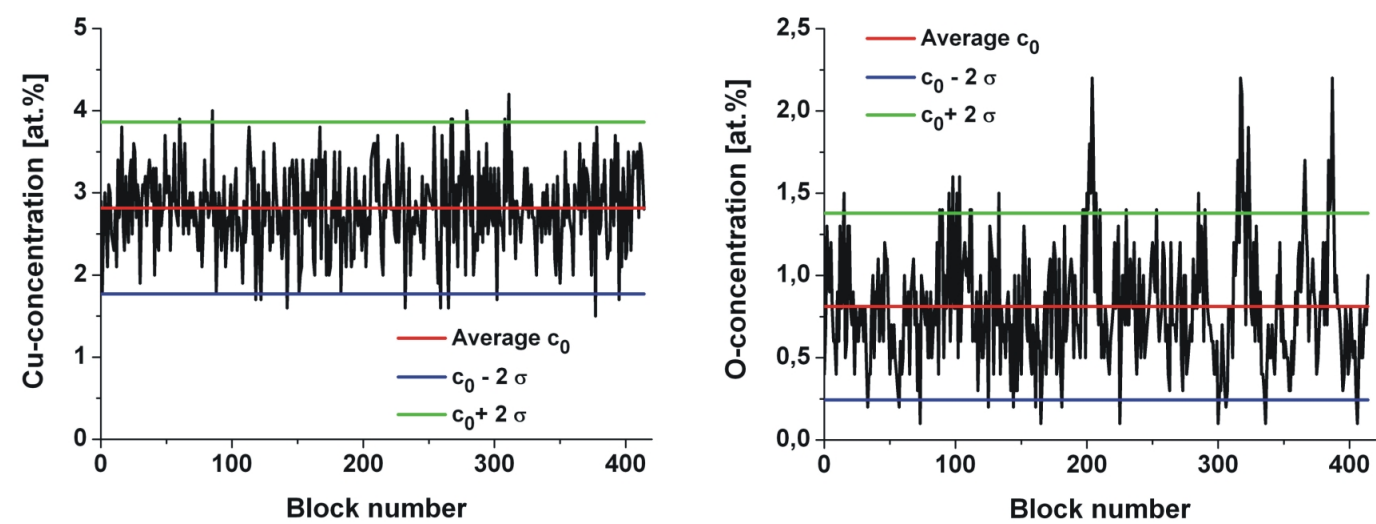

Figure 4.51: Concentration depth profiles of Fe-2.5at.\%Cu after a) $2 \mathrm{~h}$, b) $10 \mathrm{~h}$ and c) $50 \mathrm{~h}$ derived from the whole measurement, 1000 atoms per block. Inhomogeneities are drastically reduced until a homogeneous Cu-distribution is reached. 
a)
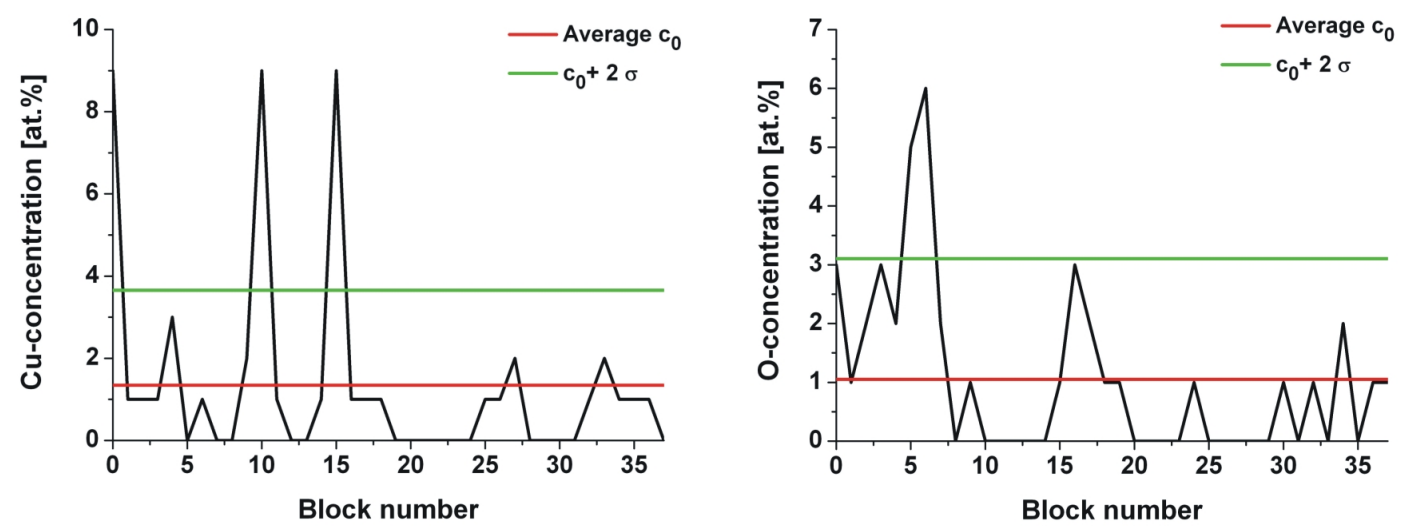

b)
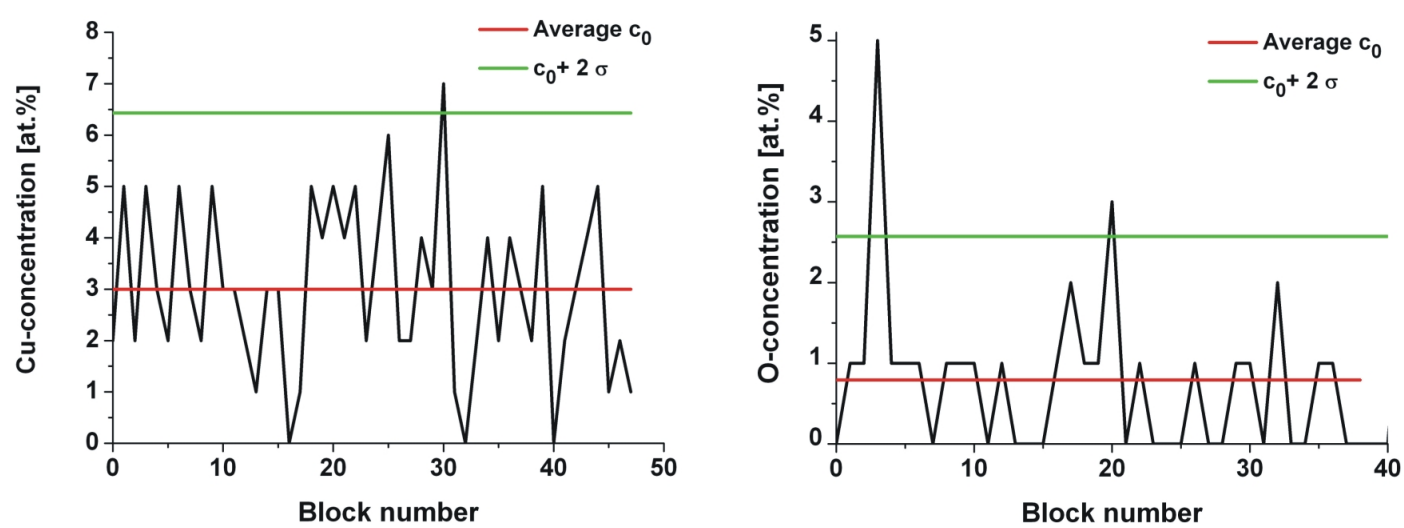

Figure 4.52: Concentration depth profiles of Fe-2.5at.\%Cu after a) $2 \mathrm{~h} \mathrm{,} \mathrm{b)} 10 \mathrm{~h}$ and c) $50 \mathrm{~h}$ derived from an analysis cylinder with a radius of $1 \mathrm{~nm}, 100$ atoms per block

can be spotted between Fig. 4.47 a) for Fe-10 at.\%Cu and Fig. 4.52 a) for Fe-2.5 at.\%Cu after $2 \mathrm{~h}$. The absolute $\mathrm{Cu}$-peak height in the former material is naturally higher as it contains the fourfold amount of $\mathrm{Cu}$ compared to the latter. But, the deviations from the $2 \sigma$-levels is $\approx 5$ at.\% for both alloys. Again, the increase in tolerance $2 \sigma$ is more then compensated by the increase in concentration variation. The most significant variation within the analysis cylinder reaches 5 at.\% after $2 \mathrm{~h}$ milling compared to 2.5 at.\% looking at the whole volume, compare Figs. 4.51 a) and 4.52 a).

Upon careful inspection of the concentration depth profiles along the analysis cylinder in Fig. 4.52 b), a difference in homogeneity between $\mathrm{Cu}$ and $\mathrm{O}$ becomes striking. The $\mathrm{Cu}-$ concentration profile stays nearly completely within the confidence intervals, whereas the $\mathrm{O}$-concentration is clearly inhomogeneous on this scale. In contrast to the development of homogeneity for the whole volume, the concentration profile of Oxygen does not smooth out with time within the analysis cylinder. 
a)

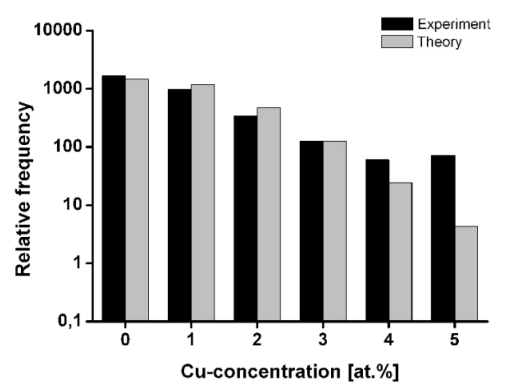

b)

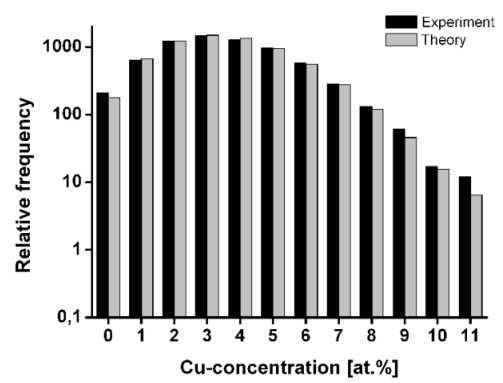

c)

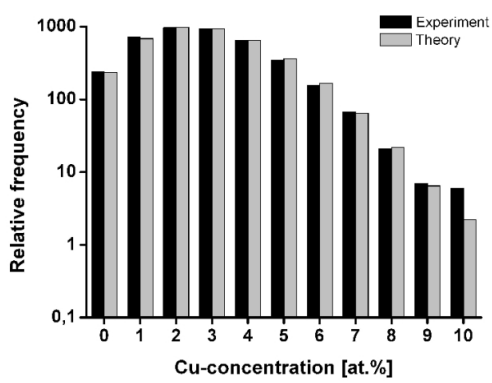

Figure 4.53: Histrograms of concentration for Fe-2.5at.\%Cu after a) $2 \mathrm{~h}$, b) $10 \mathrm{~h}$ and c) $50 \mathrm{~h}$

Consulting once again the $\chi^{2}$-tests after $2 \mathrm{~h}$ of milling, a similar situation arises for Fe-2.5 at.\%Cu as for the other Fe-rich alloys. The theoretical and experimental $\chi^{2}$-values of this sample differ greatly $\left(\chi_{\exp }^{2}=1212\right.$ and $\chi_{\text {theo }}^{2}=12.6$ ), once more resulting in a very small $\alpha$-value $\left(\alpha=5.5 \cdot 10^{-33}\right)$. Again the high concentrations are overrepresented in the experimental distribution, contributing the main fraction the the high $\chi^{2}$-value (cf. Fig. $4.53 \mathrm{a}$ )).

But, as in $\mathrm{Fe}-5$ at.\%Cu the $\mathrm{Cu}$-concentration frequency distribution of $\mathrm{Fe}-2.5 \mathrm{at} . \% \mathrm{Cu}$ after $10 \mathrm{~h}$ gives a completely different impression, as can be seen in Fig. $4.53 \mathrm{~b}$ ). Only the slightest deviations occur between the experimental and theoretical curve. This fact can be quantified by the two $\chi^{2}$-values of $\chi_{\exp }^{2}=19.5$ and $\chi_{\text {theo }}^{2}=21.0$. In this case the experimental value falls below the theoretical one as for Fe- 5 at.\% after the same milling time, resulting in an $\alpha$-value of $\alpha=0.05$. As mentioned above, the calculated $\alpha$-value exceeds 0.05 , so the observation is consistent with the null hypothesis and the null hypothesis cannot be rejected at the $5 \%$ level. By means of ball milling a state could finally be reached within this immiscible system, which is fully homogeneous on the nano-scale.

The calculated $\alpha$-value exceeds 0.05 , so the observation is consistent with the null hypothesis we fail to reject the null hypothesis at the $5 \%$ level.

After the prolonged milling time of $50 \mathrm{~h}$ the histogram of $\mathrm{Cu}$-concentrations in Fig. $4.53 \mathrm{c}$ ) looks alike the one for $10 \mathrm{~h}$ milling time. The overall agreement between experimental and theoretical curve is even slightly better for this milling time. Only minor deviation for high concentrations can be found anymore yielding a $\chi_{\exp }^{2}=10.8$ compared to $\chi_{\text {theo }}^{2}=19.7$. The theoretical value being two times the experimental one results in $\alpha=0.38$. So the statistical certainty of the material after $50 \mathrm{~h}$ being homogenous is significantly increased compared to the state after $10 \mathrm{~h}$ milling.

Figure 4.54 shows the graphical compilation of the average $\alpha$-values of Fe-2.5 at.\% Cu after 


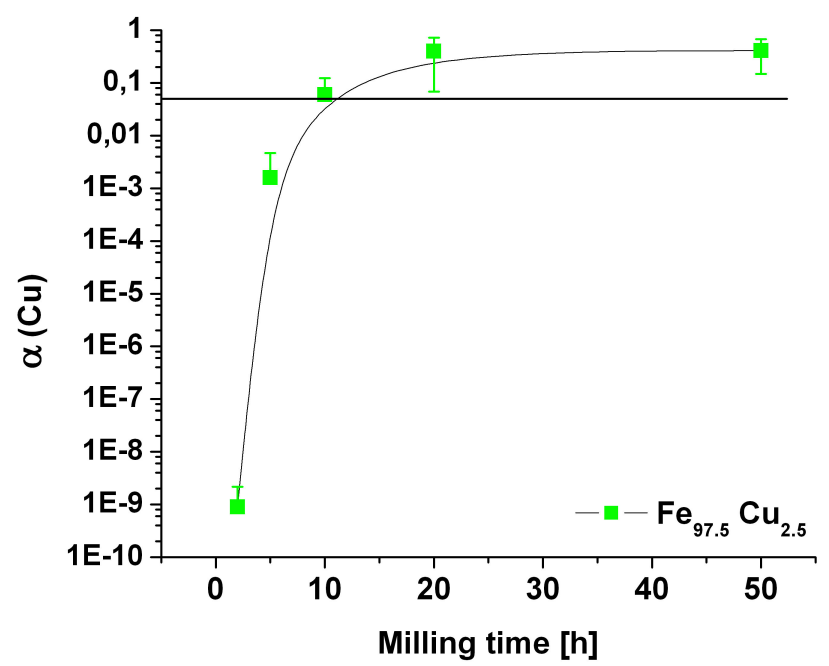

Figure 4.54: Level of significance $\alpha$ versus milling time for Fe-2.5at.\%Cu

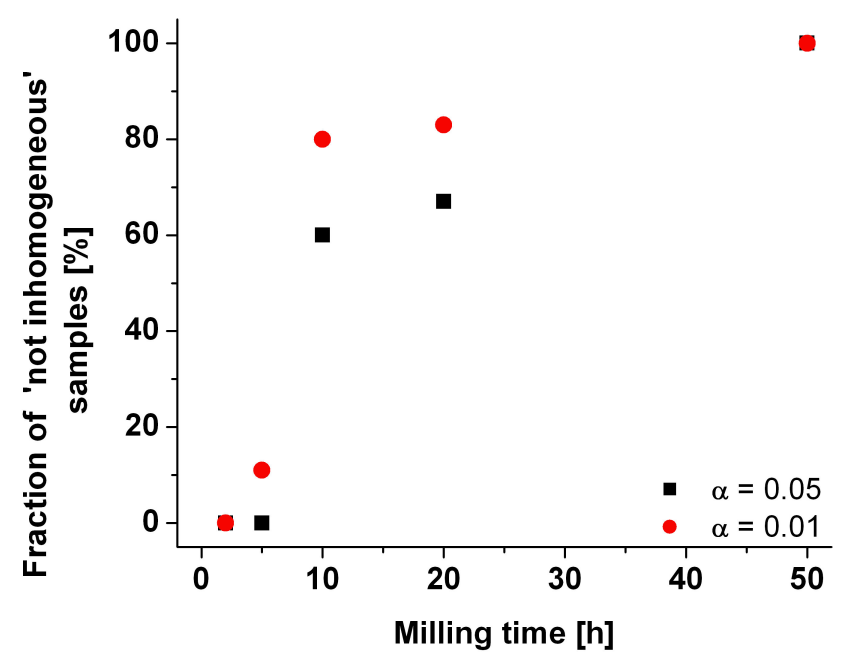

Figure 4.55: Fraction of 'not inhomogeneous samples' versus milling time (Fe-2.5 at.\% Cu): It strongly depends on the chosen level of significance $\alpha$ 
various milling times. All Fe-rich alloys show the same trend in the behavior of $\alpha$ with milling time. As for $\mathrm{Fe}-10$ at.\% $\mathrm{Cu}$ and $\mathrm{Fe}-5$ at.\% $\mathrm{Cu}$ the initial steep increase in the average $\alpha$ levels out for higher milling times.

As the statistical evaluation of homogeneity is complex, special care has to be taken when making statements on homogeneity. The level of significance of $\alpha=0.05$ is arbitrarily set. Looking at the different samples measured for milling time of $\mathrm{Fe}-2.5$ at.\% $\mathrm{Cu}$ the percentage of 'not inhomogeneous samples' strongly depends on the chosen level of significance, as can be seen in Fig. 4.55. While at a level of $\alpha=0.05$ for all measurements of Fe-2.5 at.\% Cu after $5 \mathrm{~h}$ the null hypothesis of homogeneity has to be rejected, at a level of $\alpha=0.01$ for one out of nine samples the null hypothesis of homogeneity cannot be rejected.

This one measurement has an $\alpha$-value of 0.01 . If $0.01 \leq \alpha_{\exp }<0.05$, the hypotheses is rejected at level $\alpha=0.05$ whereas it is not rejected at level $\alpha=0.01$. So the difference between the samples can be regarded as significant but not as highly significant.

Nevertheless, independent on the chosen level of significance the fraction of 'not inhomogeneous samples' increases with milling time. In other words, the homogeneity of the samples of Fe-2.5 at.\% Cu increases with milling time. This composition reaches a fully homogeneous state after milling times of $10 \mathrm{~h}$ or more.

\subsection{Thermal characterisation by DSC}

Finally the thermal stability for the composition Fe-10at.\%Cu after $10 \mathrm{~h}$ milling time was investigated exemplarily for the ball milled Fe-rich alloys. The DSC scan revealed a sharp and strongly exothermic peak at $690 \mathrm{~K}$, followed by a less intense but as sharp peak at $850 \mathrm{~K}$ depicted in Fig. 4.56. The peak at $690 \mathrm{~K}$ results in a stored enthalpy of $5.7 \mathrm{~kJ} / \mathrm{mol}$.

This is in good agreement with the DSC experiments carried out by Eckert et. al. on Fe10at.\%Cu [Eckert93b]. They reported two exothermic maxima at 680 and $750 \mathrm{~K}$. In their case, the second maximum was not a sharp one but a broad high temperature shoulder to the pronounced peak at $680 \mathrm{~K}$. Eckert identified the first peak with an exothermic phase separation accompanied by grain growth and strain release. It yielded a stored enthalpy of $5.8 \mathrm{~kJ} / \mathrm{mol}$ for Fe-10at.\%Cu after $8 \mathrm{~h}$ milling. The difference in peak position of $10 \mathrm{~K}$ most likely stems from the different heating rates employed. While the heating rate within this work was 15 $\mathrm{K} / \mathrm{min}$ Eckert used $20 \mathrm{~K} / \mathrm{min}$. A higher heating rate results in higher peak temperatures as the response of the material takes time and thus is recorded at a later time, which is a later temperature. 


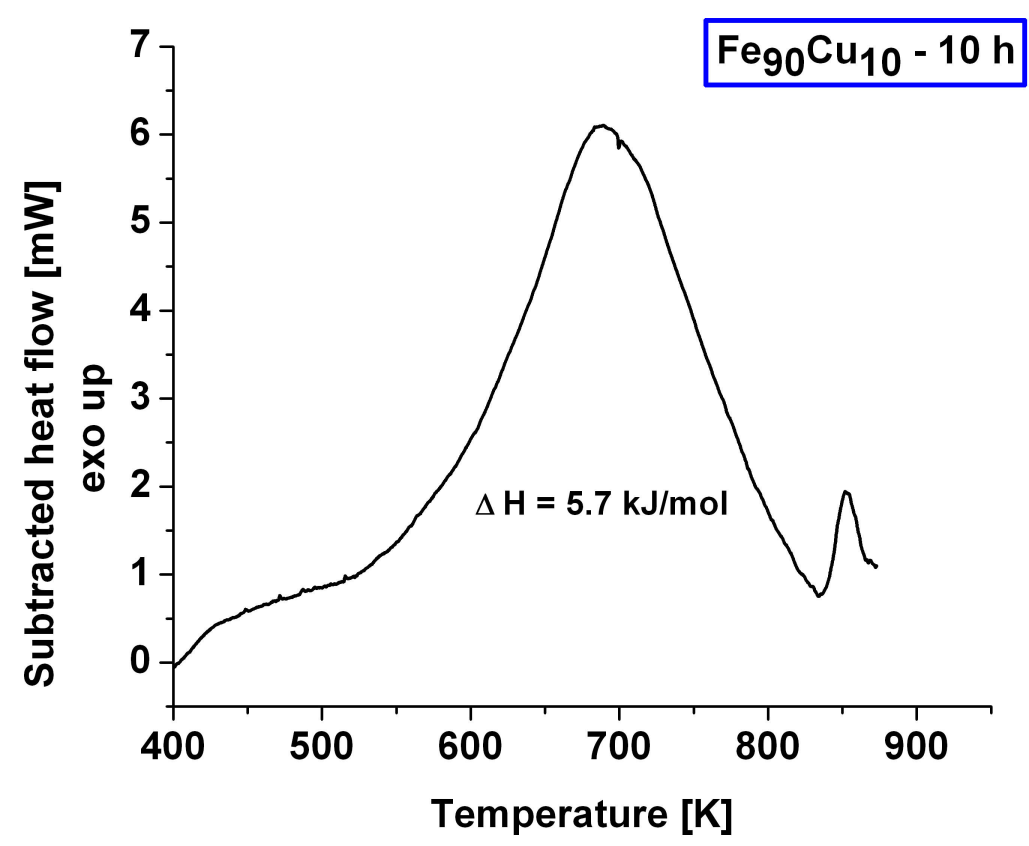

Figure 4.56: DSC trace of $\mathrm{Fe}-10 \mathrm{at} . \% \mathrm{Cu}$ after $10 \mathrm{~h}$ milling time taken at a heating rate of $15 \mathrm{~K} / \mathrm{min}$, peak temperature $690 \mathrm{~K}$

For a given composition Eckert found a maximum in stored energy depending on milling time. In the case of Fe-10at.\%Cu this maximum was reached after $8 \mathrm{~h}$ milling time. After 24 $\mathrm{h}$ milling time the stored enthalpy only amounted to $3.6 \mathrm{~kJ} / \mathrm{mol}$. The similar milling times employed in this work and Eckert's (10 h and $8 \mathrm{~h}$, respectively) yield stored enthalpies which agree very well.

Ma and co-workers also employed a SPEX shaker mill and reports a stored enthalpy of about $5 \mathrm{~kJ} / \mathrm{mol}$ after $20 \mathrm{~h}$ milling of Fe-10at.\%Cu [Ma93]. This slightly lower value agrees with both the stored enthalpy determined with this work and Eckert's finding of a maximum stored enthalpy after $8 \mathrm{~h}$ milling. 



\section{Conclusions}

The structural investigations of the ball milled Fe-Cu powders revealed the same basic features on all length scales from LOM to APT (from $1 \mathrm{~mm}$ down to some $\AA$ ).

Since not only the particle size but as well the grain size of the $\mathrm{Cu}$-rich powders rather increased then decreased with milling time, the dominating mechanism during ball milling of the $\mathrm{Cu}$-rich particles is cold-welding. Smaller particles impinging on bigger ones form mushroom shaped particles, which further are subjected to collisions with the container walls and balls. This leads to smooth and big particles, but prevents complete mixing even on the $\mu \mathrm{m}$ scale.

Unfortunately, this incomplete physical mixing yields lacking chemical mixing on the nmscale. The different field evaporation behaviour of the two components in addition to this poor miscibility results in frequent failure of the specimen during APT analysis. Future attempts using laser assisted APT systems might help to overcome the unfavourable mechanical properties of the $\mathrm{Cu}$-rich powders.

In contrast to the Cu-rich-powders, the structural evolution of the Fe-rich powders was dominated by fracture. On all length scales observed, ranging from $\mu \mathrm{m}$ by means of SEM to some $\AA$ by APT, the lamellar structure of the particles was obvious. As in the Cu-rich particles the SEM images clearly hinted at the formation of the particles. But due to the lower ductility of the majority components in the Fe-rich powders, the impinging particles were not incorporated into their target particles but rather attached to their surfaces. The resulting particles were not only flat and flaky, but contained many cracks.

The lamellar structure became obvious in the TEM images (aspect ratios of 4-9) and resulted in a strongly textured material. The texture could be explained by the fundamental work of Kudjumow and Sachs in terms of the conventional rolling texture of cold rolled Fe-sheets. Furthermore the TEM images revealed a multitude of lattice defects (estimated to be near the theoretical limit), which were taken into consideration during XRD analysis as well. Comparing the grain size derived from XRD traces by means of Scherrer's formula with the strain corrected one from the Williamson-Hall analysis, the difference was striking - $D_{\text {Scherrer }}=12 \mathrm{~nm}$ 
and $\mathrm{D}_{\mathrm{WH}}=36 \mathrm{~nm}$. Scherrer's formula vastly underestimates the grain size by a factor of at least 3. This means, not only that dislocations make a relevant contribution towards the microstructural evolution of the powders, but also that the different crystallographic directions are differently populated by these dislocations. Correcting the grain size for the fully homogenous sample of $\mathrm{Fe}-2.5 \mathrm{at} . \% \mathrm{Cu}$ after $10 \mathrm{~h}$ milling time by the above mentioned factor of 3 , a lower limit for the grain size can be given as $27 \mathrm{~nm}$. Thus, the corrected value for grain size obtained from the Williamson-Hall analysis rules out the model by Veltl et al. [Velt191] and Eckert et al. [Eckert93b] as they observed the formation of grain sizes in the range of 10-20 nm preceding substantial alloying.

As a quantitative analysis of the dislocation density was not possible here, the work of Shen was consulted as the parameters obtained within the Williamson-Hall analysis were compatible with the ones derived within this work. Their analysis yielded a dislocation density of $\rho \approx 6 \cdot 10^{16}$ near the theoretical limit as already suggested by the TEM micrographs. The strong deformation of the single grains also became apparent in the Nelson-Riley analysis of the XRD traces. A lattice expansion of $0.4 \%$ after $10 \mathrm{~h}$ milling time was found within this work for Fe10 at.\% Cu. Transferring the trend line of lattice expansion from Eckert's low energy ball milled material to the results of high energy ball mills obtained by Hong and within this work, the same behaviour of lattice expansion with milling time can be observed in both regimes. The model by Eckert et al. [Eckert93a], [Eckert93b] comprised not only the reduced grain size as a driving force for the mixing of immiscible elements, but also an increased dislocation density. This part of the model can fully be supported since the dislocation density found in our material was very high as described above.

Generally, the minority component of immiscible systems is expected to segregate to the grain boundaries upon the formation of nanocrystalline structures, thus decreasing the grain boundary energy and stabilising the structures by minimising the driving force for grain growth. Within this work, the distribution of the minority component in the Fe-rich powders $(\mathrm{Cu})$ was found to reach a homogeneous state for two of the three investigated compositions. For Fe-5at.\%Cu and $\mathrm{Fe}-2.5 \mathrm{at} . \% \mathrm{Cu}$ the difference between the binomial distribution and the experimentally determined distribution was no more significant after $10 \mathrm{~h}$ of milling time. So instead of segregation in these nanocrystalline material true alloying was found for the minority component.

The part of the segregating species may have been transferred to the impurity element oxygen. Though it only amounted to $\approx 1$ at.\%, apart from Fe and $\mathrm{Cu}$ it was the only element that could be assigned to the mass peaks in the spectra of the APT analyses. Not even debris (Cr, V, 
$\mathrm{C}, . .$.$) from the stainless steel vial and balls was detected. Evaluating the concentration depth$ profiles of oxygen for Fe-2.5at.\%Cu after $2 \mathrm{~h}$ milling time, the distances between neighbouring $\mathrm{O}$-enrichments ranged from 3 to $20 \mathrm{~nm}$. Comparing these values with the grain size of this sample determined by means of the Williamson-Hall procedure $(12 \mathrm{~nm})$, it can be assumed that the oxygen is located at the grain boundaries reducing their energy and stabilising the nanostructure. At the same time this rules out the model for enforced mixing in immiscible elements by Yavari [Yavari92a], as the oxygen is not distributed evenly in the grains and does not form a ternary mixture with a negative heat of mixing. Additionally, to significantly alter the thermodynamic properties of the alloy, a larger amount of oxygen would have been expected.

In contrast to the model proposed by Yavari et al. [Yavari92b], Gente et al. [Gente93] did not suggested incoherent by rather coherent interfaces for the $\mathrm{Cu}$-Co system. The chemical contribution of the interface could lead to an increase of the free energy of the composite above that of the solid solution. This model finally could even apply to systems with a positive energy of mixing. The gradual dissolution of the respective minority component as found in the analysis by APT strongly supports the assumption of coherent interfaces. This gradual dissolution was manifest in the low but varying $\mathrm{Cu}$-concentrations (around the nominal composition) and especially in the fact, that within more then 60 measurements only once a region of $100 \% \mathrm{Cu}$ was found.

The question of the underlying processes of mechanical alloying cannot finally be answered here, but certain models can be excluded to contribute to the enhanced miscibility in the FeCu system investigated within this work and others can be supported.

Not only the results on lattice expansion, grain size and morphology are very well comparable to the results of earlier studies, but also the DSC curves agree quantititatively as well as qualitatively. The detailed processes leading to the heat release peak at $690 \mathrm{~K}$ associated with a stored enthalpy of $5.7 \mathrm{~kJ} / \mathrm{mol}$ will be investigated with the scope of further studies. 



\section{Summary and Outlook}

The homogeneity of Fe-Cu powder particles with various nominal compositions was successfully investigated at the nano-scale applying different analysis methods. These powder particles were prepared by means of a mechanical alloying procedure utilising planetary ball mill equipment. Samples required for analysis with TEM and APT were prepared from native powders without any further consolidation or compaction procedure by means of FIB machining. Thus, any artefacts of further preparation steps could be excluded. The results within this investigation confirm some of the findings of previous publications. Some studies by other authors were limited to examining the alloying process by XRD on a much coarser scale and found the material to be homogenously alloyed.

In contrast to the usual findings, for the short milling times, TEM and APT results reveal a significant volume fraction of the powder to remain inhomogeneous, or in other words, non uniformly alloyed on the nano-scale.

This result is more pronounced for the $\mathrm{Cu}$-rich side of the phase diagram. Whereas the Ferich powders show a smoothing of these heterogeneities on the nano-scale in particular for high $\mathrm{Cu}$ contents. Even on the nano-scale full homogeneity can be achieved for this material as shown in this contribution by APT, resulting in an extended miscibility region in comparison to the equilibrium phase diagram.

In addition, owing to the outstanding chemical analysis power of APT, not only the dissolution of $\mathrm{Cu}$ into the Fe-matrix can be followed on the nano-scale but also the distribution of any kind of additional impurities may be exposed and detected on this scale. The distribution of oxygen - as the main impurity - does not smooth out as the $\mathrm{Cu}$-concentration, most probably because of grain boundary segregation of this solute. For the investigated milling times and compositions the results clearly exclude the formation of any phases in this system, in particular inter-metallics.

Generally too little $\mathrm{Cu}$ is detected as this ductile material tends to stick to the vial and balls. Aiming for a certain $\mathrm{Cu}$-content in the final powders this has to be kept in mind. Increasing the amount of $\mathrm{Cu}$ initially weighed in or drastically cooling down the whole mill, thus reducing 
the ductility of $\mathrm{Cu}$, might offer the solution to this problem. However, the latter approach will significantly alter not only the ductility of $\mathrm{Cu}$ but also the deformation mechanisms taking place.

Especially for the $\mathrm{Cu}$-rich powders future investigations will be necessary to improve the statistics on Fe-enrichments. Theses studies might significantly be facilitated by using a laser assisted APT system, thus reducing the mechanical stresses on the specimens. Concerning the Fe-rich specimens, wide angle APT measurements may be of interest. Since newly developed algorithms for these systems allow for determining size, boundaries and orientations of single grains, such wide angle APT measurements might enable a correlation between the $\mathrm{O}$-enrichments and the grain structure. Thus, it could finally be clarified whether the oxygen segregates to the grain boundaries or not.

Another issue to be addressed to be within the scope of future studies is the thermal stability of these powders with particular focus on the processes of decomposition and any interreaction on this nano-scale. 


\section{List of Figures}

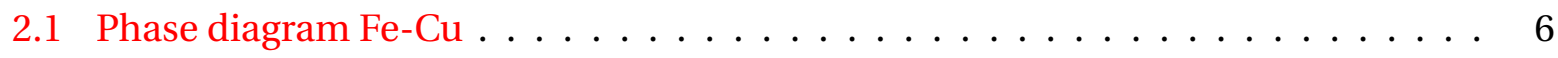

2.2 Phase boundaries for Fe-Cu alloys processed by different methods . . . . . . . . 7

2.3 Ball-powder-ball collision of powder mixture during mechanical alloying . . . . . 9

3.1 Preparation of a TEM lamella by means of FIB . . . . . . . . . . . . . . . . 15

3.2 Final shape of a TEM lamella produced by FIB machining . . . . . . . . . . . . 16

3.3 Adapting the lift-out technique . . . . . . . . . . . . . . . 17

3.4 Schematic view of the Field Ion Microsope . . . . . . . . . . . . . . . . 22

3.5 Processes at the tip apex . . . . . . . . . . . . . . . . . 23

3.6 Indexed field ion micrograph of (011) oriented $\mathrm{W}, 70 \mathrm{~K}$ and $12 \mathrm{kV} \ldots \ldots$. . . . . 24

3.7 Schematic view of the Tomographic Atom Probe . . . . . . . . . . . . . . 25

3.8 Schematic drawing of an FIIT analysis . . . . . . . . . . . . . . . . 28

3.9 DSC samples . . . . . . . . . . . . . . . . . . . . . . . . . . 29

3.10 Design of a DSC instrument . . . . . . . . . . . . . 30

4.1 LOM:Cross-sectional slices through a Cu-5at.\%Fe particle $(5 \mathrm{~h}) \ldots \ldots$. . . . . . 32

4.2 Proposed alloying mechanism . . . . . . . . . . . . . . . . 33

4.3 Particle size versus milling time: $\mathrm{Cu}$-rich $\ldots \ldots \ldots \ldots$

4.4 Particle size versus milling time: Fe-rich . . . . . . . . . . . . . . . . 34

4.5 LOM: Fe-rich alloys . . . . . . . . . . . . . . . . . . . . . 35

4.6 SEM: comparison Cu-rich and Fe-rich powders . . . . . . . . . . . . . 36

4.7 X-ray patterns of $\mathrm{Cu}-10$ at.\% $\mathrm{Fe} \ldots \ldots \ldots \ldots \ldots$

4.8 Grain size derived from the X-ray patterns for $\mathrm{Cu}$-rich alloys . . . . . . . . . . . 38

4.9 X-ray patterns of $\mathrm{Fe}-10$ at.\% $\mathrm{Cu} \ldots \ldots \ldots$. . . . . . . . . . . . 39

4.10 Grain size derived from the x-ray patterns for Fe-rich alloys . . . . . . . . . . 39

4.11 XRD patterns of $\mathrm{Fe}-2.5$ at.\% $\mathrm{Cu}$, embedded samples . . . . . . . . . . . . 41

4.12 Lattice expansion in \% versus milling time for the Fe-rich alloys . . . . . . . . . 41 
4.13 Lattice parameter versus milling time for $\mathrm{Fe}-10$ at.\% $\mathrm{Cu}$, this work compared to the literature . . . . . . . . . . . . . . . . . . . . 42

4.14 Williamson-Hall-plot for Fe-2.5 at.\% Cu after $2 \mathrm{~h} \ldots \ldots$. . . . . . . . . . . . 43

4.15 Modified Williamson-Hall-plot for Fe-2.5 at.\% Cu after $2 \mathrm{~h} \ldots \ldots$. . . . . . . . . 44

4.16 Modified Williamson-Hall-plot for $\mathrm{Cu}-2.5$ at.\% Fe . . . . . . . . . . . . . . 45

4.17 Grain size vs. milling time for Cu-rich alloys employing Ungár's method . . . . 46

$4.18 \mathrm{BF}$ images of $\mathrm{Cu}-2.5 \mathrm{at} . \% \mathrm{Fe} \ldots \ldots \ldots \ldots \ldots \ldots$

4.19 TEM BF images of the surface region of the Fe-rich particles . . . . . . . . . 49

4.20 TEM BF images of the inner region of the Fe-rich particles . . . . . . . . . . . . 50

4.21 Aspect ratio versus milling time for all Fe-rich samples . . . . . . . . . . . . . 51

4.22 TEM BF images at higher magnification of Fe-10at.\% Cu . . . . . . . . . . 52

4.23 TEM of a coarser grained surface region in Fe-10at.\%Cu after $2 \mathrm{~h}$ milling . . . . 54

4.24 TEM of a typical region in Fe-2.5at.\%Cu after $2 \mathrm{~h} \ldots \ldots \ldots 5$

4.25 TEM: grain size versus milling time for all Fe-rich samples . . . . . . . . . . . . 56

$4.26 \mathrm{SAD}$ pattern of Fe-2.5at.\%Cu after $2 \mathrm{~h} \ldots \ldots \ldots \ldots \ldots$

4.27 Debye-Scherrer photograph of cross rolled Fe sheet . . . . . . . . . . . . 58

$4.28 \mathrm{SAD}$ pattern of Fe-2.5at.\%Cu after $2 \mathrm{~h}$, texture components marked . . . . . . 59

4.29 FIM of Cu-2.5at.\%Fe after (a) $10 \mathrm{~h}$ and (b) $2 \mathrm{~h} \ldots \ldots \ldots$. . . . . . . . 61

4.30 FIIT:Iso brightness surfaces in $\mathrm{Cu}-2.5$ at.\% Fe after $2 \mathrm{~h}$ milling . . . . . . . . . . 62

4.31 FIM of Fe-10at.\%Cu after 10 h . . . . . . . . . . . . . . . . . . . . 62

$4.32 \mathrm{Cu}$-rich samples: Average measured Cu-concentration versus milling time . . . . 64

4.33 Fe-rich samples: Average measured $\mathrm{Cu}$-concentration versus milling time . . . . 65

4.34 Cu-rich samples: Average measured O-concentration versus milling time . . . . . 66

4.35 Fe-rich samples: Average measured O-concentration versus milling time . . . . . 66

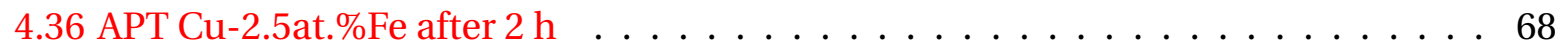

4.37 APT Cu-2.5at.\%Fe after $2 \mathrm{~h}$ - grain boundary . . . . . . . . . . . . . 69

4.38 APT Cu-2.5at.\%Fe after $10 \mathrm{~h} \ldots \ldots \ldots \ldots \ldots \ldots$

4.39 APT reconstruction of Fe-10at.\%Cu after $2 \mathrm{~h}$ and $10 \mathrm{~h} \ldots \ldots \ldots \ldots$

4.40 Concentration depth profiles of Fe-10at.\%Cu after $2 \mathrm{~h}, 3.5 \mathrm{~h}$ and $10 \mathrm{~h} \ldots \ldots$

4.41 Concentration depth profiles of Fe-10at.\%Cu after $2 \mathrm{~h}$ and $10 \mathrm{~h}$. . . . . . . 74

4.42 Histrograms of concentration for Fe-10at.\%Cu after $2 \mathrm{~h}, 3.5 \mathrm{~h}$ and $10 \mathrm{~h} \quad \ldots . .75$

4.43 Level of significance $\alpha$ versus milling time for $\mathrm{Fe}-10$ at. $\% \mathrm{Cu} \ldots \ldots$

4.44 Strongly imohomogeneous region in Fe-10 at.\%Cu after $5 \mathrm{~h}$. . . . . . . . . . 77

4.45 APT reconstruction of Fe-5at.\%Cu after $2 \mathrm{~h}$ and $10 \mathrm{~h} \ldots \ldots \ldots$ 
4.46 Concentration depth profiles of Fe-5at.\%Cu after $2 \mathrm{~h}, 5 \mathrm{~h}$ and $10 \mathrm{~h} \ldots \ldots$. . . . 79

4.47 Concentration depth profiles of Fe-5at.\%Cu after $2 \mathrm{~h}, 5 \mathrm{~h}$ and $10 \mathrm{~h} \ldots \ldots$

4.48 Histrograms of concentration for Fe-5at.\%Cu after $2 \mathrm{~h}, 5 \mathrm{~h}$ and $10 \mathrm{~h} \ldots \ldots 1$

4.49 Level of significance $\alpha$ versus milling time for $\mathrm{Fe}-5$ at. $\% \mathrm{Cu} \ldots \ldots$. . . . . . . 82

4.50 APT reconstruction of Fe-2.5at.\%Cu after $2 \mathrm{~h}$ and $50 \mathrm{~h} \ldots \ldots$. . . . . . 83

4.51 Concentration depth profiles of Fe-2.5at.\%Cu after $2 \mathrm{~h}, 10 \mathrm{~h}$ and $50 \mathrm{~h} \quad \ldots$. . . 84

4.52 Concentration depth profiles of Fe-2.5at.\%Cu after $2 \mathrm{~h}, 10 \mathrm{~h}$ and $50 \mathrm{~h}$. . . . . 85

4.53 Histrograms of concentration for Fe-2.5at.\%Cu after $2 \mathrm{~h}, 10 \mathrm{~h}$ and $50 \mathrm{~h}$. . . . 86

4.54 Level of significance $\alpha$ versus milling time for $\mathrm{Fe}-2.5 \mathrm{at} . \% \mathrm{Cu} \ldots \ldots$. . . . . . . 87

4.55 Fraction of 'not inhomogeneous samples' versus milling time (Fe-2.5 at.\% Cu) . . 87

4.56 DSC trace of Fe-10at.\%Cu after $10 \mathrm{~h} \ldots \ldots \ldots$. . . . . . . . 89 



\section{Bibliography}

[Al-Kassab03] T. Al-Kassab, H. Wollenberger, G. Schmitz, and R. Kirchheim. Tomography by Atom Probe Field Ion Microscopy. In: F. Ernst and M. Rühle (Editors), High Resolution Imaging and Spectrometery of Materials, 274-320. Springer, Berlin Heidelberg New York (2003). 22, 25

[Ashcroft02] N. Ashcroft and N. Mermin. Solid State Physics. Thomson Learning (2002). 43

[Atkins02] P. W. Atkins and J. de Paula. Atkin's Physical Chemistry. Oxford University Press, Oxford, 7th edition (2002). 5

[Benjamin74] J. S. Benjamin and T. E. Volin. Mechanism of Mechanical Alloying. Metallurgical Transactions, volume 5(8), 1929 (1974). 10

[Benjamin90] J. S. Benjamin. Mechanical alloying - a perspective. Metal Powder Report, volume 45, 122 (1990). 5

[Bronštein00] I. N. Bronštein, K. A. Semendjajew, G. Musiol, and H. Mühlig. Taschenbuch der Mathematik. Harri Deutsch, Frankfurt am Main, 5th edition (2000). 27

[Cahn83] R. Cahn and P. Haasen (Editors). Physical Metallurgy. Elsevier (1983). 68

[Choi07a] P.-P. Choi, T. Al-Kassab, Y.-S. Kwon, J.-S. Kim, and R. Kirchheim. Application of Focused Ion Beam to Atom Probe Tomography Specimen Preparation from Mechanically Alloyed Powders. Microscopy and Microanalysis, volume 13(05), 347 (2007). 4, 14

[Choi07b] P.-P. Choi, Y.-S. Kwon, J.-S. Kim, and T. Al-Kassab. Transmission electron microscopy and atom probe specimen preparation from mechanically alloyed powder using the focused ion-beam lift-out technique. J Electron Microsc (Tokyo), volume 56(2), 43 (2007). 4, 14 
[Cullity67] B. Cullity. Elements of X-Ray diffraction. Addison-Wesley, 3rd edition (1967). 17

[Eckert92] J. Eckert, J. C. Holzer, C. Krill, III, and W. L. Johnson. Structural and Thermodynamic Properties of Nanocrystalline fcc Metals prepared by Mechanical Attrition. Journal of Materials Research, volume 7(7), 1751 (1992). 40

[Eckert93a] J. Eckert, J. C. Holzer, and W. L. Johnson. Thermal stability and grain growth behavior of mechanically alloyed nanocrystalline $\mathrm{Fe}-\mathrm{Cu}$ alloys. Journal of Applied Physics, volume 73(1), 131 (1993). 6, 10, 11, 92

[Eckert93b] J. Eckert, J. C. Holzer, C. E. Krill, III, and W. L. Johnson. Mechanically driven alloying and grain size changes in nanocrystalline $\mathrm{Fe}-\mathrm{Cu}$ powders. Journal of Applied Physics, volume 73(6), 2794 (1993). 10, 11, 40, 42, 68, 88, 92

[Eckert95] J. Eckert. Relationships governing the grain size of nanocrystalline metals and alloys. Nanostructured Materials, volume 6(1-4), 413 (1995). 9

[FEI06] FEI. xT Nova NanoLab User's Manual, 5th edition (2006). 14

[Gente93] C. Gente, M. Oehring, and R. Bormann. Formation of thermodynamically unstable solid solutions in the $\mathrm{Cu}$-Co system by mechanical alloying. Phys. Rev. B, volume 48(18), 13244 (1993). 11, 93

[Giannuzzi99] L. A. Giannuzzi and F. A. Stevie. A review of focused ion beam milling techniques for TEM specimen preparation. Micron, volume 30(3), 197 (1999). 14

[Giannuzzi04] L. A. Giannuzzi and F. A. Stevie. Intoduction to focused ion beams: Instrumentation, Theory, Techniques and Practice. Springer (2004). 14

[Gleiter89] H. Gleiter. Nanocrystalline materials. Progress in Materials Science, volume 33(4), 223 (1989). 7

[Gorria06] P. Gorria, D. Martínez-Blanco, R. Iglesias, S. Palacios, M. Pérez, J. Blanco, L. F. Barquín, A. Hernando, and M. González. Magneto-volume effects in Fe-Cu solid solutions. Journal of Magnetism and Magnetic Materials, 
volume 300(1), 229 (2006). The third Moscow International Symposium on Magnetism 2005. 4

[Grovenor87] C. R. M. Grovenor, A. Cerezo, J. A. Liddle, and S. G. D. W. Pulsed laser atom probe analysis of semiconductor materials. In: C. A.G. and P. D. Augustus (Editors), Microscopy of Semiconducting Materials, number 87 in Institute of Physics Conference Series, 665-674. Institute of Physics, Bristol Philadelphia (1987). 16

[Haasen94] P. Haasen. Physikalische Metallkunde. Springer, Berlin, 2nd edition (1994). 5

[Heinrich05] A. Heinrich. Zum Einfluss der elastischen Verzerrungsenergie auf die Frühstadien der Entmischung von Cu-2at.\%Co. Ph.D. thesis, Göttingen (2005). 28, 29

[Hong98] L. Hong and B. Fultz. Two-phase coexistence in Fe-Cu alloys synthesized by ball milling. Acta Materialia, volume 46(8), 2937 (1998). 6, 7, 42

[Koch93] C. Koch. The synthesis and structure of nanocrystalline materials produced by mechanical attrition: A review. Nanostructured Materials, volume 2(2), 109 (1993). 8

[Koch97] C. Koch. Synthesis of nanostructured materials by mechanical milling: problems and opportunities. Nanostructured Materials, volume 9(1-8), 13 (1997). 8

[Krengel00] U. Krengel. Einführung in die Wahrscheinlichkeitstheorie und Statistik. Vieweg, Braunschweig Wiesbaden, 5th edition (2000). 27

[Lee98] P.-Y. Lee, J.-L. Yang, and H.-M. Lin. Amorphization behaviour in mechanically alloyed Ni-Ta powders. Journal of Materials Science, volume 33(1), 235 (1998). 10

[Ma93] E. Ma, E. Atzmon, and F. E. Pinkerton. Thermodynamic and magnetic properties of metastable $\mathrm{Fe}_{\mathrm{x}} \mathrm{Cu}_{100-\mathrm{x}}$ solid solutions formed by mechanical alloying. Journal of Applied Physics, volume 74(2), 955 (1993). 6, 89 
[Massalski90] T. B. Massalski. Binary Alloy Phase Diagrams. ASM International, Materials Park, 2nd edition (1990). 6

[Miedema76] A. R. Miedema. Heat of Formation of Alloys. Philips Technical Review, volume 36(8), 217 (1976). 5

[Miller96] M. K. Miller, A. Cerezo, M. G. Hetherington, and G. D. W. Smith. Atom Probe Field Ion Microscopy. Oxford University Press, Oxford (1996). 4, 16, 22,23

[Miller03] M. K. Miller, B. D. Wirth, and G. R. Odette. Precipitation in neutronirradiated Fe- $\mathrm{Cu}$ and $\mathrm{Fe}-\mathrm{Cu}-\mathrm{Mn}$ model alloys: a comparison of APT and SANS data. Materials Science and Engineering A, volume 353(1-2), 133 (2003). 47 th International Field Emission Symposium. 65

[Miller05] M. Miller, K. Russell, and G. Thompson. Strategies for fabricating atom probe specimens with a dual beam FIB. Ultramicroscopy, volume 102(4), 287 (2005). 4, 14

[Orloff02] J. Orloff, M. Utlaut, and L. Swanson. High resolution focused ion beams: FIB and its applications. Kluwer Academic/ Plenum Publishers (2002). 14, 65

[Scherrer18] P. Scherrer. Bestimmung der Größe und der inneren Struktur von Kolloidteilchen mittels Röntgenstrahlen. Nachrichten von der Gesellschaft der Wissenschaften zu Göttingen, volume 1918, 98 (1918). 18

[Schmidt07] M. Schmidt. Zur Homogenität von kugelgemahlenen Kupfer-EisenLegierungen. Master's thesis, Göttingen (2007). 6, 26, 31, 32, 33, 38, 45, $46,47,48,60,61,62,63,68,69,70$

[Shen05]

T. D. Shen, R. B. Schwarz, and J. D. Thompson. Soft magnetism in mechanically alloyed nanocrystalline materials. Phys. Rev. B, volume 72(1), 014431 (2005). 45, 53

[Stokes44] A. R. Stokes and A. J. C. Wilson. The diffraction of X rays by distorted crystal aggregates - I. Proceedings of the Physical Society, volume 56(3), 174 (1944). 18 
[Suryanarayana90] C. Suryanarayana and F. H. Froes. Nanocrystalline titanium-magnesium alloys through mechanical alloying. Journal of Materials Research, volume 5(9), 1880 (1990). 10

[Suryanarayana00] C. Suryanarayana and C. Koch. Nanocrystalline materials - Current research and future directions. Hyperfine Interactions, volume 130(1), 5 (2000). 7

[Suryanarayana01] C. Suryanarayana. Mechanical alloying and milling. Progress in Materials Science, volume 46(1-2), 1 (2001). 3, 8, 9, 10, 37

[Uenishi91] K. Uenishi, K. Kobayashi, K. Ishihara, and P. Shingu. Formation of a supersaturated solid solution in the Ag- $\mathrm{Cu}$ system by mechanical alloying. Materials Science and Engineering: A, volume 134, 1342 (1991). Proceedings of the Seventh International Conference on Rapidly Quenched Materials. 48

[Ungár99a] T. Ungár, I. Dragomir, Á. Révész, and A. Borbély. The contrast factors of dislocations in cubic crystals: the dislocation model of strain anisotropy in practice. Journal of Applied Crystallography, volume 32(5), 992 (1999). 20

[Ungár99b] T. Ungár and G. Tichy. The Effect of Dislocation Contrast on X-Ray Line Profiles in Untextured Polycrystals. physica status solidi (a), volume 171(2), 425 (1999). 19, 20

[Velt191] G. Veltl, B. Scholz, and H.-D. Kunze. Amorphization of $\mathrm{Cu}-\mathrm{Ta}$ alloys by mechanical alloying. Materials Science and Engineering: A, volume 134, 1410 (1991). Proceedings of the Seventh International Conference on Rapidly Quenched Materials. 10, 92

[Vurpillot04] F. Vurpillot, M. Gilbert, and B. Deconihout. The Field Ion Microscope: A 3D Microscope. Oral Presentation at IFES (2004). 28

[Warren69] B. E. Warren. X-Ray Diffraction. Addison-Wesley (1969). 17

[Wassermann39] G. Wassermann. Texturen metallischer Werkstoffe. Springer, Berlin (1939). 57,58 
[Wille06a]

[Wille06b]

[Wille08]

[Wille09]

[Williamson53]

[Wolde-Giorgis05]

[Yavari92a]

[Yavari92b]
C. Wille. Untersuchungen an nanokristallinem Ni und Co-3,2at. \%P mit der Feldionenbild- und Atomsondentomographie. Master's thesis, Göttingen (2006). 28, 29

C. Wille, T. Al-Kassab, A. Heinrich, and R. Kirchheim. Nanostructured materials studied by means of the computed field ion image tomography (CFIIT). In: 19th International Vacuum Nanoelectronics Conference and 50th International Field Emission Symposium, 17-18. IEEE (2006). 29

C. Wille, T. Al-Kassab, M. Schmidt, P.-P. Choi, and Y.-S. Kwon. Homogeneity of mechanically alloyed nano-crystalline $\mathrm{Fe}-\mathrm{Cu}$-powders. International Journal of Materials Research, volume 99(5), 541 (2008). 63

C. Wille, T. Al-Kassab, P.-P. Choi, Y.-S. Kwon, and R. Kirchheim. An assessment of the homogeneity of nano-crystalline $\mathrm{Fe}-\mathrm{Cu}$ powders as studied by means of APT. Ultramicroscopy, volume 109(5), 599 (2009). IFES 2008, Proceedings of the 51th International Field Emission Symposium. 63

G. Williamson and W. Hall. X-ray line broadening from filed aluminium and wolfram. Acta Metallurgica, volume 1(1), 22 (1953). 19, 43

D. Wolde-Giorgis. Korngrenzsegregation in Silber-Nickel und KupferWismut Legierungen. Ph.D. thesis, Göttingen (2005). 16

A. R. Yavari and P. J. Desré. Thermodynamics and Kinetics of Amorphisation during Mechanical Alloying. Materials Science Forum, volume 88 90, 43 (1992). 11, 93

A. R. Yavari, P. J. Desré, and T. Benameur. Mechanically driven alloying of immiscible elements. Phys. Rev. Lett., volume 68(14), 2235 (1992). 11, 93 


\section{Publications}

C.Wille, T. Al-Kassab, P.-P. Choi, Y.-S. Kwon, and R. Kirchheim. An assessment of the homogeneity of nano-crystalline Fe-Cu powders as studied by means of APT. Ultramicroscopy, volume 109(5), 599 (2009). IFES 2008, Proceedings of the 51th International Field Emission Symposium.

C. Wille, T. Al-Kassab, M. Schmidt, P.-P. Choi, and Y.-S. Kwon. Homogeneity of mechanically alloyed nano-crystalline Fe-Cu-powders. International Journal of Materials Research, volume 99(5), 541 (2008). 



\section{Danksagung}

An erster Stelle gilt mein großer Dank Herrn Prof. Dr. Reiner Kirchheim für die Ermöglichung einer Dissertation am Institut für Materialphysik, seine stetige Bereitschaft auf meine Fragen einzugehen und die sich daraus entwickelnden anregenden und hilfreichen Diskussionen.

Herrn Prof. Dr. Tala'at Al-Kassab danke ich für fortwährende intensive Betreuung (auch über Kontinente hinweg) und sein stets offenes Ohr bei allen experimentellen, theoretischen und sonstigen Problemen, für alle Diskussionen, Gespräche und Ermutigungen sowie für die großen Freiräume bei der Ausgestaltung dieser Arbeit.

Herrn Prof. Dr. Hans Christian Hofsäss danke ich für die Übernahme des Korreferates.

Herrn Dipl.-Phys. Malte Schmidt danke ich für die gute Zusammenarbeit und seinen Kampf mit den störrischen Cu-Pulvern. Für die Bereitstellung der kugelgemahlenen Proben im Rahmen des binationalen DFG-Projektes zwischen den Universitäten Göttingen und Ulsan danke ich Herrn Prof. Dr. Young-Soon Kwon und Herrn Dr. Pyuck-Pa Choi. Außerdem danke ich Herrn Dr. Pyuck-Pa Choi für seine Diskussionsbereitschaft und schnelle Hilfe per Email.

Den ehemaligen und jetzigen Mitgliedern der FIM-Gruppe danke ich für Hilfe und Unterstützung und Diskussionen: Dr. Alexander Heinrich, M.Sc. Michael Sobol, Dipl.-Phys. Torben Boll, Dipl.-Phys. Thomas Rademacher, Dipl.-Phys. Thomas Kresse und Dr. Carsten Nowak.

Allen Technikern der Werkstätten, besonders Herrn Tobias Schulz, danke ich für die unbürokratische Hilfe bei Problemen. Herrn Volker Radisch sei insbesondere für die schlanken TEM-Lamellen gedankt. Herrn Dr. Peter-Joachim Wilbrandt und Frau Dr. Christine Borchers danke ich für die Diskussionen und Hilfen zur Interpretation der TEM-Bilder.

Meinen Freunden möchte ich für die regelmäßigen Hinweise auf ein Leben außerhalb der Physik danken, darunter seien Johanna, Christian, Michi und nicht zuletzt Sönke besonders erwähnt für die diversen Tassen Kaffee und Portionen Eis am „See “.

Sönke danke ich außerdem für die regelmäßigen Aufmunterungen aus Ferne und Nähe, für den Rückhalt, der er mir gegeben hat, und nicht zuletzt für seine Geduld mit mir und Latex.

Meinen Eltern danke ich für die Ermöglichung meines Studiums und die vorbehaltlose Unterstützung in allen Lebenslagen. 


\section{Lebenslauf}

\section{Persönliche Daten}

Name Catharina Gabriele Wille

Geburtsort Buchholz i.d.N.

Geburtstag 27. Oktober 1980

Nationalität deutsch

\section{Schulbildung}

$07 / 2000$

Allgemeine Hochschulreife, Albert-Einstein-Gymnasium (Buchholz i.d.N.)

\section{Studium}

10/2000-01/2006 Physikstudium an der an der Georg- August- Universität zu Göttingen

10/2002 Vordiplomprüfung im Fach Physik

08/2004-01/2006 Diplomarbeit am Institut für Materialphysik zum Thema „Untersuchungen an nanokristallinem Ni und Co-3,2at\%P mit der Feldionenbild- und Atomsonden-Tomographie“

01/2006 Diplomprüfung im Fach Physik

seit 3/2006 Wissenschaftliche Mitarbeiterin an der Georg- August- Universität zu Göttingen am Institut für Materialphysik in der Arbeitsgruppe von Prof. Dr. R. Kirchheim 